\title{
A goodness-of-fit test for the ordered stereotype model
}

\author{
Daniel Fernández ${ }^{* \dagger}$ and Ivy Liu
}

\begin{abstract}
This paper presents a new goodness-of-fit test for an ordered stereotype model used for an ordinal response variable. The proposed test is based on the well-known Hosmer-Lemeshow test and its version for the proportional odds regression model. The latter test statistic is calculated from a grouping scheme assuming that the levels of the ordinal response are equally spaced which might be not true. One of the main advantages of the ordered stereotype model is that it allows us to determine a new uneven spacing of the ordinal response categories, dictated by the data. The proposed test takes the use of this new adjusted spacing to partition data. A simulation study shows good performance of the proposed test under a variety of scenarios. Finally, the results of the application in two examples are presented. Copyright () 2016 John Wiley \& Sons, Ltd.
\end{abstract}

Keywords: Goodness-of-fit; Hosmer-Lemeshow test, ordinal data; ordered stereotype model; uneven spacing

\section{Introduction}

An ordinal variable is one with a categorical data scale, which describes order, and where the distinct levels of such a variable differ in degree of dissimilarity more than in quality [1]. This is different from nominal variables which vary in quality, not in quantity, and thus, the order of listing the categories is irrelevant. Stevens [2] called a scale ordinal if 'any order-preserving transformation will leave the scale form invariant'. Examples of ordinal variables are the measures of the effectiveness of a new drug ('low', 'medium', or 'high'), the pain scale (Figure 1), and the Likert scale responses in a questionnaire might be ('disagree', 'neither agree nor disagree', or 'agree').

There are a variety of approaches to the modelling of ordinal data that properly respect the ordinal nature of the data, without the assumption that the data are continuous. Liu and Agresti [3] and Agresti [1] described various proportional odds version models using adjacent-categories logits, cumulative logits [4], and continuation-ratio logits [5]. In the literature, often, a proportional odds model refers to the one using cumulative logits, which is the most commonly used model for an ordinal response variable. The proportional odds structure makes a strong assumption on common odds ratios, and this may be inadequate for some data. Alternatively, a partial proportional odds model [6] allows non-proportional odds for some or all covariates, but the model might contain many parameters especially when there are many response categories. This paper focuses on an ordered stereotype model [7], which is more flexible than the models with the proportional odds structure as a result of adding additional score parameters. In terms of model parsimony, it lies between proportional odds and partial proportional odds models.

In practice, researchers are interested to know how well the model fits. Even for a model with many predictors, there is still no guarantee that the model provides a good fit. Most of the goodness-of-fit test statistics do not focus on how accurate the predictions are, but on whether, we can make a better model by using a more complex form [8]. Hosmer et al. [9] comprehensively reviewed goodness-of-fit tests for binary logistic regression models including the popular Hosmer and Lemeshow test [10]. However, there are few methods of goodness-of-fit for ordinal data. For a binary response case, summary measures of fit are functions of the differences between observed and fitted values. The fitted values in a logistic

School of Mathematics and Statistics, Victoria University of Wellington, Wellington, New Zealand

*Correspondence to: Daniel Fernández, School of Mathematics and Statistics, Victoria University of Wellington, PO Box 600,

Wellington 6140, New Zealand.

†E-mail:dfdez23@hotmail.com 


\section{Statistics}

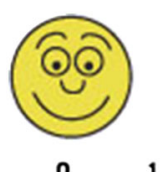

$\mathbf{0}$

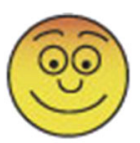

2

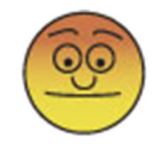

3

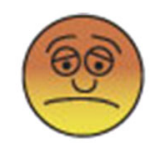

5

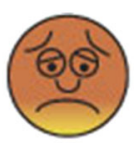

7

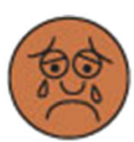

10

Figure 1. This scale measures a patient's pain intensity in which 0 means no pain and 10 means extremely painful. Pain scales are based on self-report, observational, or physiological data.

regression model can be calculated for each covariate pattern based on the estimated probability for that pattern [9, Ch. 5]. The Pearson $\chi^{2}$ and the deviance statistics are two classical summary measures across different covariate patterns. Both test statistics require a large sample with few covariate patterns so that the asymptotic theory holds. For an ordinal response case with $q$ categories, each response is considered as a vector with multinomial data, where the $k$ th element refers to the 'yes' or 'no' response to the $k$ th category, $k=1, \ldots, q-1$ [11, Ch. 3]. The fitted values of response categories are calculated from the estimated probability vector in a model. Then, the goodness-of-fit of the model may be checked through the Pearson $\chi^{2}$ and the deviance statistics. Both statistics become the summary measures of the differences between observed and fitted values across different covariate patterns and response categories. Similar to the binary case, the asymptotic theory fails for a large number of covariate patterns.

Pulkstenis and Robinson [12] developed a modified version of the Pearson $\chi^{2}$ and the deviance statistics for a proportional odds model that includes continuous covariates and a few categorical covariates. Fagerland et al. [13] proposed a generalization of the binary case to the multinomial logistic regression model, but this model does not have an intrinsic constraint to be used with ordinal data. Lipsitz et al. [14] proposed a goodness-of-fit test for a wide range of ordinal regression models, but it has the drawback that it is not always computable for small samples. Fagerland and Hosmer [15] extended the binary logistic regression case [9] to develop a goodness-of-fit test for proportional odds models. From the simulation study, Fagerland and Hosmer [15] showed that although the tests by Lipsitz et al. [14] and PulkstenisRobinson [12] had greater power to detect a missing interaction term, the Fagerland-Hosmer method was able to detect a broad range of lack of fit. Lin and Chen [16] gave a goodness-of-fit test for proportional odds models by applying a non-parametric local linear smoothing technique. In addition to the test statistics, Liu et al. [17] developed a graphical method for assessing the adequacy of the functional form of a covariate in a proportional odds model using cumulative sums of residuals. Furthermore, $\mathrm{Li}$ and Shepherd [18] proposed a new residual for regression models of ordinal outcomes, where the new residual is a single value per subject and contains directional information between the observed value and the fitted distribution.

To the best of our knowledge, there is no approach specifically developed for testing the fit of an ordered stereotype model. The purpose of this paper is to fill this lack and to present a new goodness-of-fit test for this model. The plan of the paper is as follows. Section 2 defines and describes the ordered stereotype model. The new goodness-of-fit test is given in Section 3, along with the way to derive the test statistic. Section 4 presents simulation studies to investigate the performance of the new test statistic for the null distribution and power. We also compare the proposed method with the Fagerland-Hosmer method [15]. Two real-life examples of the application are given in Section 5. We conclude with a discussion in Section 6.

\section{The ordered stereotype model}

Let $Y_{i}$ be an ordinal response with $q$ categories (e.g., strongly agree, agree, neutral, disagree, and strongly disagree) for observation $i$, where $i=1, \ldots, n$. The ordered stereotype model [7] for the probability that $Y_{i}$ takes the category $k$ is characterized by the following log odds:

$$
\log \left(\frac{P\left[Y_{i}=k \mid \boldsymbol{x}_{i}\right]}{P\left[Y_{i}=1 \mid \boldsymbol{x}_{i}\right]}\right)=\alpha_{k}+\phi_{k} \boldsymbol{\beta}^{\prime} \boldsymbol{x}_{i}, \quad i=1, \ldots, n, \quad k=2, \ldots, q,
$$

where the inclusion of the following monotone non-decreasing constraint

$$
0=\phi_{1} \leq \phi_{2} \leq \cdots \leq \phi_{q}=1
$$


ensures that the response $Y_{i}$ is ordinal ([7]). The vector $\boldsymbol{x}_{i}$ is a set of predictor variables for observation $i$, which can be categorical or continuous, and the vector of parameters $\beta$ represents the effects of $\boldsymbol{x}_{i}$ on the $\log$ odds for the category $k$ relative to the baseline category of $Y_{i}$. The model treats the first category as the baseline category, the parameters $\left\{\alpha_{2}, \ldots, \alpha_{q}\right\}$ are the cut points, and $\left\{\phi_{1}, \phi_{2}, \ldots, \phi_{q}\right\}$ are the parameters that can be interpreted as the 'scores' for the categories of the response variable $Y_{i}$. We restrict $\alpha_{1}=\phi_{1}=0$ and $\phi_{q}=1$ to ensure identifiability. With this construction, the response probabilities are as follows:

$$
\theta_{i k}=P\left[Y_{i}=k \mid \boldsymbol{x}_{i}\right]=\frac{\exp \left(\alpha_{k}+\phi_{k} \boldsymbol{\beta}^{\prime} \boldsymbol{x}_{i}\right)}{\sum_{\ell=1}^{q} \exp \left(\alpha_{\ell}+\phi_{\ell} \boldsymbol{\beta}^{\prime} \boldsymbol{x}_{i}\right)} \quad \text { for } k=1, \ldots, q
$$

where the probability for the baseline category, as defined in (3), satisfies

$$
\theta_{i 1}=1-\sum_{\ell=2}^{q} P\left[Y_{i}=\ell \mid \boldsymbol{x}_{i}\right]
$$

and therefore, because $\alpha_{1}=\phi_{1}=0$, this probability can be defined as

$$
\theta_{i 1}=\frac{1}{1+\sum_{\ell=2}^{q} \exp \left(\alpha_{\ell}+\phi_{\ell} \boldsymbol{\beta}^{\prime} \boldsymbol{x}_{i}\right)} \text {. }
$$

Greenland [19] stated that the stereotype model is appropriate when the progression of the response variable occurs through various stages. An advantage of the stereotype model is that it is more parsimonious than the baseline-category logit model that has the form $\boldsymbol{\alpha}_{k}+\boldsymbol{\beta}_{\boldsymbol{k}}^{\mathbf{\prime}} \boldsymbol{x}_{i}$ on the right-hand side of model (1). On the other hand, the ordered stereotype model is more flexible than adjacent-categories logits models with proportional odds structure [1, Section 4.3.4] as a result of the $\left\{\phi_{k}\right\}$ parameters. Agresti [1] (Chapter 4) showed that the stereotype model is equivalent to the proportional odds version of the adjacent-categories logit model, when the scores $\left\{\phi_{k}\right\}$ are equally spaced. Although the model has advantages, it is not as popular as the proportional odds model, because the parameters are more difficult to estimate due to the intrinsic nonlinearity which arises from the product of parameters in the predictor. The parameter estimates may be calculated by the standard maximum likelihood (ML) method [1] by imposing the monotone non-decreasing constraint (2) through the reparametrization described in Fernández et $a l$. [20]. This paper obtains the ML estimates using the quasi-Newton method provided as an option of optim() in the statistical package R 3.2.2 [21].

\section{The proposed test statistic}

The proposed test statistic follows closely the test proposed in [15] for the proportional odds model. The main difference with our test is that we take the advantage of score parameters $\left\{\phi_{k}\right\}$ in the ordered stereotype model to determine a new spacing of the ordinal response categories. The steps to construct the proposed test are as follows:

(1) Calculate the estimated probabilities $\widehat{\theta}_{i k}$ (Eq. (3)) for each observation $i=1, \ldots, n$ and response category $k=1, \ldots, q$.

(2) Compute the weighted score for each observation:

$$
s_{i}=\sum_{k=1}^{q} v_{k} \times \hat{\theta}_{i k}, \quad i=1, \ldots, n,
$$

where $v_{1}=1, v_{q}=q$, and $v_{k}=1+(q-1) \times \hat{\phi}_{k}$. Note that the $\left\{v_{k}\right\}$ in the range of $[1, q]$ is the rescaled ordinal scores for the response categories, calculated from the score parameter estimates $\left\{\hat{\phi}_{k}\right\}$ in $[0,1]$.

(3) Replace the observed response $\left\{y_{i}\right\}$ for each observation by its corresponding rescaled ordinal scores $\left\{v_{k}\right\}$, denoted by $\left\{\hat{y}_{i}\right\}$. For example, $\hat{y}_{i}=v_{k}$ if $y_{i}=k$. Because of the nature of ordinal stereotype models, the spacing information between response categories is better captured by $\left\{v_{k}\right\}$. As a result, the equal spacing between categories is removed by the new fitted spacing. 
(4) Compute the deviances for each observation: $d_{i}=s_{i}-\hat{y}_{i}(i=1, \ldots, n)$.

(5) Sort the $n$ observations ascending by $\left\{d_{i}\right\}$.

(6) Create a first partition into $g_{1}$ groups of the data, such that each group $\ell$ contains $n_{\ell}=n / g_{1}$ observations $\left(\ell=1, \ldots, g_{1}\right.$ and $\left.n=n_{1}+n_{2}+\ldots+n_{g_{1}}\right)$. For instance, if $g_{1}=2$, the data are divided into two portions in which each portion contains $50 \%$ of the observations. As a result of this step, the data are grouped according to the level of deviations. This is favorable to produce similar groups of observations based on their quality of fit (deviance).

(7) For each $g_{1}$ group, we sort the corresponding $\left\{n_{\ell}, \ell=1, \ldots, g_{1}\right\}$ observations ascending by the weighted scores $\left\{s_{i}\right\}$.

(8) For each $g_{1}$ group, we create a second partition into $g_{2}$ sub-groups based on the weighted scores $\left\{s_{i}\right\}$, such that each sub-group contains $\left\{n_{\ell} / g_{2}, \ell=1, \ldots, g_{1}\right\}$ observations. Sorting according to the weighted score follows closely the sorting used in [15]. At the end of this step, we have partitioned the data into $G=g_{1} \times g_{2}$ groups.

(9) Cross-classify the observations according to the $G$ groups and the ordinal response categories to create a $G \times q$ contingency table. The observed frequencies $\left\{o_{g k}\right\}$ and the estimated expected frequencies $\left\{e_{g k}\right\}$ under the model are defined as follows:

$$
o_{g k}=\sum_{v \in \Upsilon_{g}} I\left[y_{v}=k\right] \quad \text { and } \quad e_{g k}=\sum_{v \in \Upsilon_{g}} \hat{\theta}_{v k}, \quad \text { for } g=1, \ldots, G, \quad k=1, \ldots, q,
$$

where $\Upsilon_{g}$ denotes the set of indices of the observations in group $g$ and $I[A]$ is a binary indicator that takes value 1 if $A$ is true and 0 otherwise. Regarding the issue concerning the size of the estimated frequencies in order to hold the $\chi^{2}-$ approximation, we follow the suggestion of [14] that all the expected frequencies should be greater than 1 and at least $80 \%$ of them should be greater than 5 .

(10) Compute the Pearson $\chi^{2}$ statistics $S_{g_{1}, g_{2}}$ as

$$
S_{g_{1}, g_{2}}=\sum_{g=1}^{G} \sum_{k=1}^{q} \frac{\left(o_{g k}-e_{g k}\right)^{2}}{e_{g k}}
$$

where $G=g_{1} \times g_{2}$. Fagerland et al. [13] posited that on the basis of the empirically proven distribution of the test statistic for binary logistic regression [10], they expect that a multinomial test follows a chi-squared distribution with $(G-2)(q-1)$ degrees of freedom. In the case of the proportional odds model, Fagerland and Hosmer [15] stated that although the overall sums of the estimated and observed frequencies are equal, the sum of the estimated frequencies are not equal to the observed in each of the $q$ levels of the ordinal response. Therefore, the statistic is expected to follow a chisquared distribution with $(G-2)(q-1)+(q-2)$ degrees of freedom in order to reflect this and the fact that one additional degree of freedom is lost due to the ordering constraint. As the nature of the stereotype model is the same as the proportional odd model, we follow [15] and posit that the $S_{g_{1}, g_{2}}$ test statistic follows a $\chi^{2}$ distribution with $(G-2)(q-1)+(q-2)$ degrees of freedom when the fitted model is correct.

The proposed two-stage grouping scheme uses the deviances $\left\{d_{i}\right\}$ and the weighted scores $\left\{s_{i}\right\}$. The motivation of using the deviances is due to a disadvantage of Hosmer-Lemeshow tests where in the grouping process, one may miss an important deviation from fit. We deliberately divide groups by the deviances to detect the lack of fit more easily than the traditional Hosmer-Lemeshow tests. We recommend to use $g_{1}=2$ that divides the observations into two portions according to the deviations. The simulation studies in the next section investigate properties of the test statistic numerically.

\section{Simulation studies}

The performance of the proposed test statistics $\left(S_{g_{1}, g_{2}}\right)$ is evaluated in Sections 4.1 and 4.2 in which the null distribution and the power of the test statistic are assessed. The results of FH test statistic [15] are also given in both cases. Although the FH test was proposed for examining goodness-of-fit for the proportional odds regression model, it could be used in the same way as the test was described but using the fitted probabilities from the ordered stereotype model. The $S_{g_{1}, g_{2}}$ and FH test statistics have the same null chi-squared distribution when the same number of partitioning groups $(G)$ is used. 


\subsection{Assessing the rejection of the null distribution}

We conduct a simulation study in a diverse range of the scenarios to evaluate the asymptotic chi-squared approximations in the upper tail for the proposed test statistic. We simulated data from the ordered stereotype model (1) varying the response categories $(q=3,4,5)$, the sample size $(n=100,200,400,800)$, and the covariates (Uniform, Normal, and/or Bernoulli distributions). Table I shows a summary of the true parameters for the model, where the score parameters $\left\{\phi_{k}\right\}$ were assigned to be equally spaced and the true parameters $\left\{\alpha_{k}, \boldsymbol{\beta}\right\}$ were chosen to avoid highly unbalanced frequencies in the response categories.

We then calculated both $S_{g_{1}, g_{2}}$ and FH test statistics using different numbers of partitioning groups $(G=6,8,10)$ when the model (1) that contains covariates described in each scenario was fitted. The fitted model is indeed the correct model. Each simulation scenario is specified by a combination of response categories, sample size, partitioning groups, and covariates. For each case, we generated 10,000 data sets (replicates). Notice that the ML parameter estimates were obtained based on a comprehensive search over different starting points to avoid finding only a local maximum. Particularly, the optim() algorithm was repeated 10 times with random starting points, and the best ML estimates (those that led to highest log-likelihood value) were kept. We have run experiments testing up to 100 random starting points, and it was sufficient with 10 repetitions to avoid convergence to local optima.

Given that the values of the covariate effect parameters $\left\{\beta_{j}\right\}$ are positive, the effect of increasing the value of the continuous covariates (Normal or Uniform variables) is to shift the distribution of the probabilities to the right, as the ordinal response increases. The effect of the Bernoulli covariates is to set scenarios so that the effect of the continuous covariates is mitigated. All the results compare the FH and $S_{g_{1}, g_{2}}$ tests throughout different scenarios. Doing this, we are not aiming to put both tests in competition as they are developed from different ordinal models (proportional odd model and stereotype model for FH and $S_{g_{1}, g_{2}}$ tests, respectively). Our goal is to check whether our test strategy in which the spacing dictated by the data and the level of deviations are included in its calculation (Section 3) has similar or slightly better results compared with the FH test. For that reason, the fitted probabilities in the FH test are computed from the stereotype model (denoted as FH-stereo in all tables).

\begin{tabular}{|c|c|c|c|c|}
\hline $\begin{array}{l}\text { Categories } \\
\text { (q) }\end{array}$ & Covariates & $\left\{\beta_{j}\right\}$ & $\left\{\alpha_{k}\right\}$ & $\left\{\phi_{k}\right\}$ \\
\hline \multirow[t]{2}{*}{3} & $\begin{array}{l}\mathcal{U}(0,10) \\
\mathcal{N}(5,3)\end{array}$ & $(0.3)$ & \multirow{2}{*}{$(0,-0.6,-1.5)$} & \multirow{2}{*}{$(0,1 / 2,1)$} \\
\hline & $\begin{array}{c}\mathcal{U}(0,10) \text { and } \operatorname{Bern}(0.5) \\
\mathcal{N}(5,3) \text { and } \operatorname{Bern}(0.5)\end{array}$ & $(0.3,0.5)$ & & \\
\hline \multirow[t]{2}{*}{4} & $\begin{array}{l}\mathcal{U}(0,10) \\
\mathcal{N}(5,3)\end{array}$ & $(0.3)$ & \multirow[t]{2}{*}{$(0,0.2,-0.8,-1.2)$} & \multirow[t]{2}{*}{$(0,1 / 3,2 / 3,1)$} \\
\hline & $\begin{array}{c}\mathcal{U}(0,10) \text { and } \operatorname{Bern}(0.5) \\
\mathcal{N}(5,3) \text { and } \operatorname{Bern}(0.5)\end{array}$ & $(0.3,0.5)$ & & \\
\hline$\Delta$ & $\begin{array}{c}\mathcal{U}(0,10) \\
\mathcal{N}(5,3) \\
\mathcal{U}(0,10) \text { and } \operatorname{Bern}(0.5) \\
\mathcal{N}(5,3) \text { and } \operatorname{Bern}(0.5)\end{array}$ & $(0.3,0.5)$ & $(0,-0.1,-0.8,-1.2,-1.6)$ & $(0,1 / 4,2 / 4,3 / 4,1)$ \\
\hline
\end{tabular}

Table II. Proportion of times (\%) that the test statistic exceeds the 100(1- 1 ) percentage point of the null chi-squared distribution when the fitted model is the true model (1), averaged over all the scenarios.

\begin{tabular}{|c|c|c|c|c|c|c|c|c|c|c|c|c|}
\hline \multirow{2}{*}{ Test } & \multicolumn{3}{|c|}{$n=100$} & \multicolumn{3}{|c|}{$n=200$} & \multicolumn{3}{|c|}{$n=400$} & \multicolumn{3}{|c|}{$n=800$} \\
\hline & $\alpha: 1 \%$ & $5 \%$ & $10 \%$ & $1 \%$ & $5 \%$ & $10 \%$ & $1 \%$ & $5 \%$ & $10 \%$ & $1 \%$ & $5 \%$ & $10 \%$ \\
\hline$S_{g_{1}, g_{2}}$ & 3.08 & 8.83 & 13.68 & 2.31 & 7.11 & 12.37 & 1.90 & 6.26 & 11.38 & 1.30 & 5.52 & 10.28 \\
\hline FH-stereo ${ }_{G}$ & 4.14 & 10.62 & 16.96 & 3.13 & 8.58 & 14.57 & 2.26 & 6.60 & 12.57 & 1.48 & 5.45 & 10.53 \\
\hline
\end{tabular}

Note: $S_{g_{1}, g_{2}}$ is the proposed test with the first partition (deviance) into $g_{1}$ and the second partition into $g_{2}$ groups; FH-stereo ${ }_{G}$ is the Fagerland-Hosmer test with the fitted probabilities from the ordered stereotype model using $G$ groups; and $G=g_{1} \times g_{2}$. 


\begin{tabular}{|c|c|c|c|c|c|c|c|c|c|c|c|c|}
\hline \multirow{2}{*}{ Test } & \multicolumn{3}{|c|}{$n=100$} & \multicolumn{3}{|c|}{$n=200$} & \multicolumn{3}{|c|}{$n=400$} & \multicolumn{3}{|c|}{$n=800$} \\
\hline & $\alpha: 1 \%$ & $5 \%$ & $10 \%$ & $1 \%$ & $5 \%$ & $10 \%$ & $1 \%$ & $5 \%$ & $10 \%$ & $1 \%$ & $5 \%$ & $10 \%$ \\
\hline$S_{2,3}$ & 2.99 & 9.04 & 13.35 & 2.22 & 7.06 & 11.97 & 1.85 & 6.23 & 11.47 & 1.24 & 5.62 & 10.35 \\
\hline FH-Stereo 6 & 3.99 & 10.45 & 17.11 & 3.05 & 8.48 & 14.29 & 2.18 & 6.73 & 12.90 & 1.46 & 5.59 & 10.59 \\
\hline$S_{2,4}$ & 3.19 & 8.19 & 14.06 & 2.38 & 6.97 & 12.76 & 1.96 & 6.12 & 11.36 & 1.33 & 5.54 & 10.35 \\
\hline FH-Stereo 8 & 4.20 & 10.75 & 16.85 & 3.11 & 8.94 & 14.87 & 2.26 & 6.68 & 12.56 & 1.39 & 5.54 & 10.49 \\
\hline$S_{2,5}$ & 3.05 & 9.25 & 13.62 & 2.33 & 7.29 & 12.39 & 1.89 & 6.42 & 11.31 & 1.33 & 5.42 & 10.13 \\
\hline $\mathrm{FH}$ Stereo $_{10}$ & 4.24 & 10.68 & 16.91 & 3.23 & 8.30 & 14.55 & 2.35 & 6.38 & 12.24 & 1.58 & 5.23 & 10.51 \\
\hline
\end{tabular}

Note: $S_{g_{1}, g_{2}}$ is the proposed test with the first partition (deviance) into $g_{1}$ and the second partition into $g_{2}$ groups; FH-Stereo ${ }_{G}$ is the Fagerland-Hosmer test with the fitted probabilities from the ordered stereotype model using $G$ groups; and $G=g_{1} \times g_{2}$.

Over 10,000 simulated data sets, we calculated the proportion of times that the test statistic exceeds the $100(1-\alpha)$ percentage point of the null chi-squared distribution for each scenario. Table II gives an overall summary of the results, and Table III breaks down the results by the number of groups $G$. Both tables show that the rejection rates of the $S_{g_{1}, g_{2}}$ test are almost always closer to the nominal level than the FH method regardless of sample sizes and $\alpha$. Although both test statistics are slightly liberal, the rejection rate of both tests is closer to the nominal level as the sample size increases. It indicates an empirical signal of good asymptotic results. The performance of the $S_{g_{1}, g_{2}}$ test with different number of groups $G$ (Table III) is very similar to the overall summary result. It implies that our test seems robust to $G$. Appendix A.1 has the full results for all scenarios in Tables AIV (for $q=3$ ), AV (for $q=4$ ), and AVI (for $q=5$ ).

Figure 2 shows histograms depicting the empirical distributions of our proposed test statistic $S_{g_{1}, g_{2}}$ when $G=g_{1} \times g_{2}=6,8,10$. The model used was model (1) with response level $q=3$ and $n=200$. We used the simulated data from the three levels of significance $(\alpha=0.01,0.05,0.10)$ in order to obtain more data to draw in the histograms, that is, we used the simulated data sets of the second set of columns when the number of groups is $G=6$ (first row), $G=8$ (third row), and $G=10$ (fifth row) in Table AIV in Appendix A.1. The figure also depicts in blue the adherence to the reference $\chi_{(G-2)(q-1)+(q-2)}^{2}$ distribution. This empirical analysis gives us good insight into the asymptotic distribution of our test statistic $S_{g_{1}, g_{2}}$, which follows the chi-square distribution we posited in Section 3 well in the three frameworks.

\subsection{Assessing the power test}

The design of simulation study to assess the power of the tests in different scenarios is similar to the evaluation of the null distribution described in the previous section. The different scenarios may be described as omission of a quadratic term in a continuous covariate (Scenario 1), omission of an interaction term between a continuous and a dichotomous covariate (Scenario 2), wrong functional form of a continuous covariate (Scenario 3), wrong ordinal response assumption, that is, nominal response variable (Scenario 4), omission of an interaction term between two dichotomous covariates (Scenario 5), omission of a continuous covariate that is independent of the other covariates (Scenario 6), and omission of an interaction term in a model with only dichotomous covariates (Scenario 7). We simulated 10,000 data sets from each of the models specified in Table IV, where $\eta_{i k}$ is the predictor function such that

$$
\theta_{i k}=\frac{\exp \left(\eta_{i k}\right)}{\sum_{\ell=1}^{q} \exp \left(\eta_{i \ell}\right)}
$$

Tables AI-AIII in Appendix A give the detail of all parameters.

For each data set, the ordered stereotype model (1) was fitted, where the predictor function is either $\eta_{i k}=\alpha_{k}+\phi_{k}(\beta x)$ or $\eta_{i k}=\alpha_{k}+\phi_{k}\left(\beta_{1} x_{1}+\beta_{2} x_{2}\right)$ depending on the scenario described in Table IV. We calculated the proportion of times that the null hypothesis was rejected at a certain fixed level of significance, over 10,000 simulated data sets for each test statistic. Table V averages power of the tests over all cases for each omission scenario. Tables AVII-AXVIII in Appendix A.2 give the detail specified by cases. 

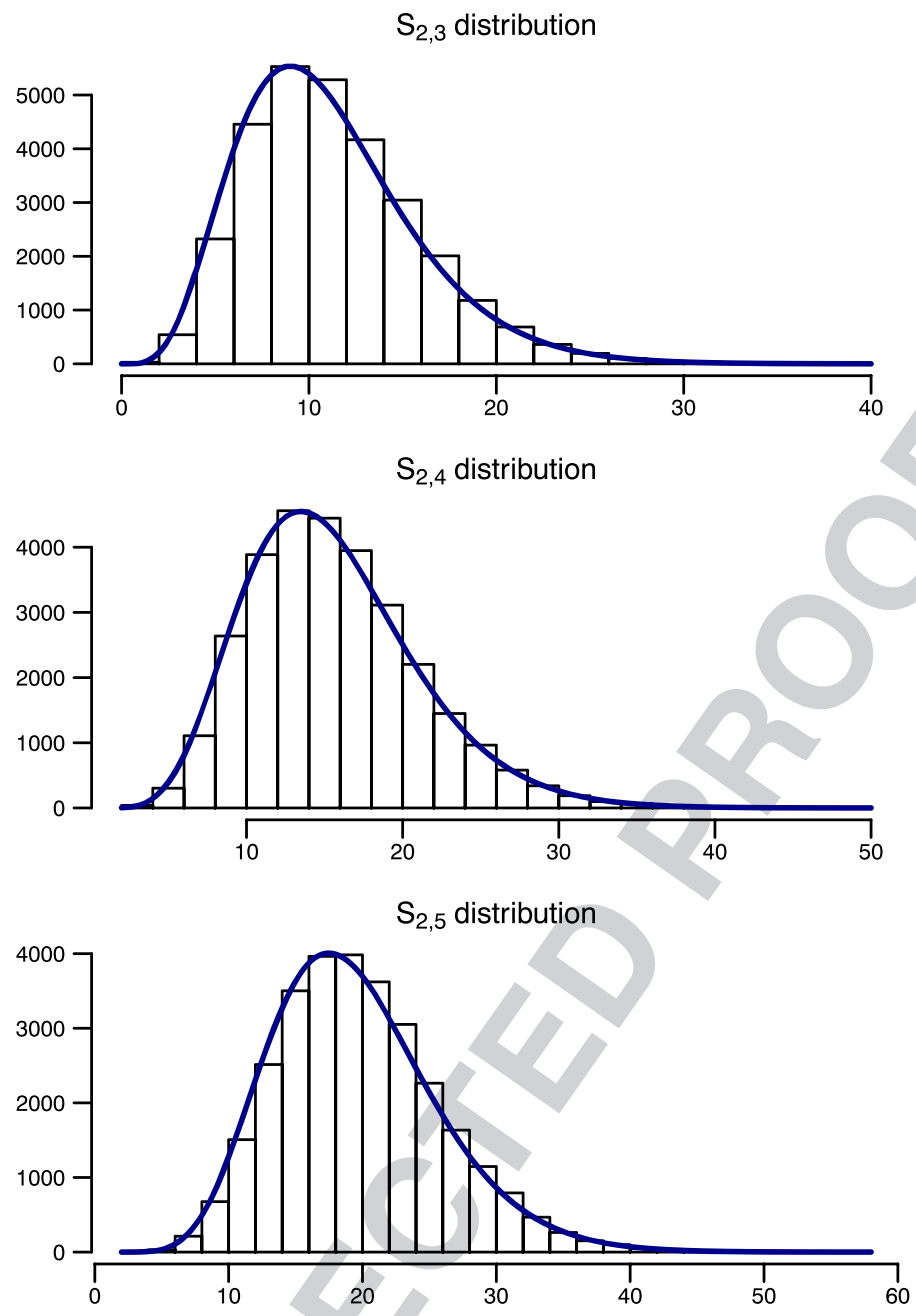

Figure 2. Histograms of the proposed test statistic $S_{g 1, g 2}$ and reference $x^{2}$ distribution curves (blue lines) with $(G-2)(q-1)+(q-2)$ degrees of freedom for the $q=3$ categorical response level model (1) with $x$ distributed as $U(0,10)$ and sample size $n=200$.

In general, power of the tests increases as the sample size increases. The $S_{g_{1}, g_{2}}$ test performs better than the FH test in terms of power for all cases except for Scenario 4 in which an ordered stereotype model was fitted when the true model is a baseline-category logit model with nominal response categories. Similar to the results in assessing the rejection of the null distribution, the power of the proposed test seems to be robust to different numbers of groups $G$ (Table VI). However, the test with $G=6$ groups had slightly higher power among the three possible numbers of groups $(G=6,8,10)$.

The results in more detail for each scenario are given in the following sections.

\section{Omission of a quadratic term in a continuous covariate}

We investigated four cases to detect a missing quadratic term in a continuous covariate, where the covariate follows either a uniform or a normal distribution. The true model for each case contains the quadratic term of the covariate. Two of the cases have an additional dichotomous covariate effect. The dichotomous covariate was generated from a Bernoulli variable with the probability of success of 0.5 . Table VII summarises the power of the test over all four cases when the number of partition groups $(G)$ is 6,8 , and 10. The proposed test $S_{g_{1}, g_{2}}$ has higher power for most of the cases compared with the FH test. The full results are given in Tables AVII-AIX in Appendix A.2.1.

\section{Omission of an interaction term between a continuous and a dichotomous covariate}

The model considered has both continuous and dichotomous covariates. Apart from the main effects, the true model includes the interaction term. We simulated the continuous covariate from either a uniform 
or a normal distribution. The dichotomous covariate was generated from a Bernoulli variable with the probability of success of 0.5 . Table VIII shows the power to detect the missing interaction term for each

\begin{tabular}{|c|c|c|c|}
\hline Scenario & Description & Covariates & Model \\
\hline 1 & $\begin{array}{l}\text { Omission of a quadratic term } \\
\text { in a continuous covariate }\end{array}$ & $\begin{array}{c}x \sim \mathcal{U}(0,10) \\
x \sim \mathcal{N}(5,3) \\
x_{1} \sim \mathcal{U}(0,10) \text { and } x_{2} \sim \operatorname{Bern}(0.5) \\
x_{1} \sim \mathcal{N}(5,3) \text { and } x_{2} \sim \operatorname{Bern}(0.5)\end{array}$ & $\begin{array}{c}\eta_{i k}=\alpha_{k}+\phi_{k}\left(0.2 x+0.1 x^{2}\right) \\
\eta_{i k}=\alpha_{k}+\phi_{k}\left(-0.25 x+0.1 x^{2}\right) \\
\eta_{i k}=\alpha_{k}+\phi_{k}\left(0.1 x_{1}+0.5 x_{2}+0.05 x_{1}^{2}\right) \\
\eta_{i k}=\alpha_{k}+\phi_{k}\left(-0.25 x_{1}+0.5 x_{2}+0.1 x_{1}^{2}\right)\end{array}$ \\
\hline 2 & $\begin{array}{l}\text { Omission of } \\
\text { an interaction term between } \\
\text { a continuous } \\
\text { and a dichotomous covariate }\end{array}$ & $\begin{array}{l}x_{1} \sim \mathcal{U}(0,10) \text { and } x_{2} \sim \operatorname{Bern}(0.5) \\
x_{1} \sim \mathcal{N}(5,3) \text { and } x_{2} \sim \operatorname{Bern}(0.5)\end{array}$ & $\begin{array}{c}\eta_{i k}=\alpha_{k}+\phi_{k}\left(0.2 x_{1}+0.5 x_{2}+0.1 x_{1} x_{2}\right) \\
\eta_{i k}=\alpha_{k}+\phi_{k}\left(-0.25 x_{1}+0.5 x_{2}+0.1 x_{1} x_{2}\right.\end{array}$ \\
\hline 3 & $\begin{array}{l}\text { Wrong functional form } \\
\text { of a continuous covariate }\end{array}$ & $\begin{array}{l}x_{1} \sim \mathcal{U}(0,10) \\
x_{2} \sim \operatorname{Bern}(0.5)\end{array}$ & $\eta_{i k}=\alpha_{k}+\phi_{k}\left(0.2 \log \left(x_{1}\right)+0.5 x_{2}\right)$ \\
\hline 4 & $\begin{array}{c}\text { Wrong } \\
\text { ordinal response } \\
\text { assumption } \\
\text { (nominal response variable) }\end{array}$ & $\begin{array}{c}x \sim \mathcal{U}(0,10) \\
x \sim \mathcal{N}(5,3) \\
x_{1} \sim \mathcal{U}(0,10) \text { and } x_{2} \sim \operatorname{Bern}(0.5) \\
x_{1} \sim \mathcal{N}(5,3) \text { and } x_{2} \sim \operatorname{Bern}(0.5)\end{array}$ & $\begin{array}{c}\eta_{i k}=\alpha_{k}+\beta_{k} x \\
\eta_{i k}=\alpha_{k}+\beta_{k} x \\
\eta_{i k}=\alpha_{k}+\beta_{1 k} x_{1}+\beta_{2 k} x_{2} \\
\eta_{i k}=\alpha_{k}+\beta_{1 k} x_{1}+\beta_{2 k} x_{2}\end{array}$ \\
\hline 5 & $\begin{array}{c}\text { Omission of } \\
\text { an interaction term } \\
\text { between two } \\
\text { dichotomous covariates }\end{array}$ & $\begin{array}{c}x_{1} \sim \mathcal{N}(5,3) \\
x_{2} \sim \operatorname{Bern}(0.5) \\
x_{3} \sim \operatorname{Bern}(0.7) / \operatorname{Bern}(0.2)\end{array}$ & $\begin{array}{c}\eta_{i k}=\alpha_{k}+\phi_{k}\left(-1.25 x_{1}+0.5 x_{2}\right. \\
\left.+2 x_{3}+1.5 x_{2} x_{3}\right)\end{array}$ \\
\hline 6 & $\begin{array}{l}\text { Omission of } \\
\text { a continuous covariate } \\
\text { (independent of } \\
\text { the other covariates) }\end{array}$ & $\begin{array}{l}x_{1} \sim \mathcal{N}(5,3) \\
x_{2} \sim \mathcal{N}(5,3) \\
x_{3} \sim \mathcal{U}(0,10)\end{array}$ & $\eta_{i k}=\alpha_{k}+\phi_{k}\left(-2.25 x_{1}+3 x_{2}+0.5 x_{3}\right)$ \\
\hline 7 & $\begin{array}{c}\text { Omission of } \\
\text { an interaction term } \\
\text { in a model with only } \\
\text { dichotomous covariates }\end{array}$ & $\begin{array}{l}x_{1} \sim \operatorname{Bern}(0.7) \text { and } x_{2} \sim \operatorname{Bern}(0.5) \\
x_{1} \sim \operatorname{Bern}(0.2) \text { and } x_{2} \sim \operatorname{Bern}(0.5)\end{array}$ & $\begin{array}{l}\eta_{i k}=\alpha_{k}+\phi_{k}\left(0.2 x_{1}+0.5 x_{2}+0.1 x_{1} x_{2}\right) \\
\eta_{i k}=\alpha_{k}+\phi_{k}\left(0.2 x_{1}+0.5 x_{2}+0.1 x_{1} x_{2}\right)\end{array}$ \\
\hline
\end{tabular}

\begin{tabular}{|c|c|c|c|c|c|c|c|c|c|c|c|c|c|}
\hline \multirow{2}{*}{ Scenario } & \multirow{2}{*}{ Test } & \multicolumn{3}{|c|}{$n=100$} & \multicolumn{3}{|c|}{$n=200$} & \multicolumn{3}{|c|}{$n=400$} & \multicolumn{3}{|c|}{$n=800$} \\
\hline & & $\alpha: 1 \%$ & $5 \%$ & $10 \%$ & $1 \%$ & $5 \%$ & $10 \%$ & $1 \%$ & $5 \%$ & $10 \%$ & $1 \%$ & $5 \%$ & $10 \%$ \\
\hline \multirow{2}{*}{ Overall } & & 1.71 & 7.45 & 13.95 & 2.20 & 8.60 & 15.60 & 2.99 & 9.98 & 17.88 & 3.79 & 12.37 & 20.80 \\
\hline & FH-Stereo ${ }_{G}$ & 1.61 & 7.15 & 12.64 & 2.04 & 8.03 & 14.62 & 2.70 & 9.23 & 16.45 & 3.46 & 10.90 & 18.65 \\
\hline \multirow{2}{*}{1} & $S_{g_{1}, g_{2}}$ & 1.82 & 6.93 & 13.60 & 2.56 & 8.80 & 16.45 & 3.18 & 10.25 & 18.90 & 3.96 & 12.84 & 22.10 \\
\hline & FH-Stereo $_{G}$ & 1.56 & 5.86 & 10.83 & 2.13 & 6.77 & 12.89 & 2.74 & 7.98 & 14.17 & 3.47 & 9.81 & 16.72 \\
\hline \multirow{2}{*}{2} & & 1.17 & 5.82 & 10.63 & 1.52 & 6.49 & 11.89 & 2.34 & 7.68 & 13.88 & 3.16 & 10.14 & 17.91 \\
\hline & FH-Stereo $_{G}$ & 0.97 & 5.09 & 9.49 & 1.34 & 6.00 & 12.03 & 1.90 & 6.96 & 13.00 & 2.49 & 8.36 & 15.38 \\
\hline \multirow{2}{*}{3} & $S_{g_{1}, g_{2}}$ & 2.76 & 11.16 & 19.01 & 3.26 & 12.04 & 20.25 & 4.57 & 14.28 & 23.73 & 5.48 & 17.40 & 26.36 \\
\hline & $\mathrm{FH}^{-S t e r e o}{ }_{G}$ & 2.45 & 10.10 & 16.05 & 2.81 & 10.69 & 17.27 & 3.72 & 12.14 & 20.20 & 4.45 & 13.46 & 22.34 \\
\hline \multirow{2}{*}{4} & $S_{g_{1}, g_{2}}$ & 1.07 & 5.88 & 12.56 & 1.47 & 7.08 & 13.82 & 1.88 & 7.70 & 15.01 & 2.55 & 9.12 & 16.84 \\
\hline & FH-Stereo $_{G}$ & 1.45 & 7.55 & 14.18 & 1.88 & 8.65 & 16.29 & 2.44 & 9.83 & 18.41 & 3.44 & 11.96 & 20.15 \\
\hline \multirow{2}{*}{5} & $S_{g_{1}, g_{2}}$ & 3.12 & 12.24 & 21.43 & 9.74 & 33.89 & 40.70 & 33.08 & 56.73 & 65.53 & 39.91 & 63.75 & 79.21 \\
\hline & FH-Stereo $_{G}$ & 1.36 & 7.70 & 14.35 & 2.91 & 12.22 & 19.34 & 10.57 & 27.05 & 26.35 & 17.02 & 35.85 & 54.67 \\
\hline \multirow{2}{*}{6} & $S_{g_{1}, g_{2}}$ & 6.79 & 19.17 & 45.78 & 7.53 & 49.16 & 52.09 & 27.42 & 65.77 & 61.41 & 30.18 & 67.88 & 66.47 \\
\hline & FH-Stereo $_{G}$ & 6.48 & 15.79 & 31.55 & 6.83 & 32.09 & 32.45 & 14.12 & 32.99 & 35.11 & 14.83 & 37.64 & 35.75 \\
\hline \multirow{2}{*}{7} & $S_{g_{1}, g_{2}}$ & 2.97 & 12.38 & 22.12 & 3.34 & 12.76 & 22.84 & 3.71 & 13.08 & 23.09 & 4.39 & 14.53 & 24.81 \\
\hline & FH-Stereo $_{G}$ & 0.74 & 4.15 & 8.93 & 1.34 & 4.44 & 9.65 & 1.55 & 5.23 & 9.98 & 1.87 & 5.51 & 10.13 \\
\hline
\end{tabular}


Table VI. Power of the test (\%) averaged over all scenarios described in Table IV, broken down by number of groups $G$.

\begin{tabular}{|c|c|c|c|c|c|c|c|c|c|c|c|c|}
\hline \multirow{2}{*}{ Test } & \multicolumn{3}{|c|}{$n=100$} & \multicolumn{3}{|c|}{$n=200$} & \multicolumn{3}{|c|}{$n=400$} & \multicolumn{3}{|c|}{$n=800$} \\
\hline & $\alpha: 1 \%$ & $5 \%$ & $10 \%$ & $1 \%$ & $5 \%$ & $10 \%$ & $1 \%$ & $5 \%$ & $10 \%$ & $1 \%$ & $5 \%$ & $10 \%$ \\
\hline$S_{2,3}$ & 2.81 & 10.72 & 21.58 & 4.16 & 18.19 & 26.06 & 10.41 & 24.26 & 32.39 & 12.75 & 28.00 & 36.87 \\
\hline $\mathrm{FH}^{- \text {Stereo }_{6}}$ & 2.21 & 8.18 & 15.58 & 2.83 & 11.64 & 17.63 & 5.53 & 14.59 & 20.51 & 7.19 & 17.85 & 25.64 \\
\hline$S_{2,4}$ & 2.82 & 10.33 & 20.15 & 4.36 & 18.56 & 24.74 & 11.78 & 25.12 & 30.85 & 13.76 & 27.77 & 36.04 \\
\hline $\mathrm{FH}_{- \text {Stereo }_{8}}$ & 2.17 & 7.83 & 14.86 & 2.78 & 11.34 & 17.16 & 5.23 & 14.51 & 19.39 & 6.71 & 17.12 & 24.72 \\
\hline$S_{2,5}$ & 2.82 & 10.48 & 20.44 & 4.08 & 19.06 & 25.51 & 10.45 & 25.84 & 31.72 & 11.91 & 28.09 & 35.81 \\
\hline FH-Stereo ${ }_{10}$ & 2.05 & 8.08 & 14.72 & 2.64 & 11.67 & 16.63 & 5.11 & 14.69 & 18.89 & 6.45 & 17.59 & 24.68 \\
\hline
\end{tabular}

Table VII. Power of the test (\%) for the detection of an omitted quadratic term in a continuous covariate (Scenario 1, Table IV) averaged over all cases, broken down by number of groups $G$.

\begin{tabular}{|c|c|c|c|c|c|c|c|c|c|c|c|c|}
\hline \multirow{2}{*}{ Test } & \multicolumn{3}{|c|}{$n=100$} & \multicolumn{3}{|c|}{$n=200$} & \multicolumn{3}{|c|}{$n=400$} & \multicolumn{3}{|c|}{$n=800$} \\
\hline & $\alpha: 1 \%$ & $5 \%$ & $10 \%$ & $1 \%$ & $5 \%$ & $10 \%$ & $1 \%$ & $5 \%$ & $10 \%$ & $1 \%$ & $5 \%$ & $10 \%$ \\
\hline$S_{2,3}$ & 2.17 & 6.97 & 13.57 & 3.11 & 9.19 & 16.24 & 3.99 & 10.74 & 19.90 & 4.59 & 13.48 & 24.00 \\
\hline FH-Stereo 6 & 1.76 & 4.96 & 8.71 & 2.18 & 5.36 & 9.72 & 2.97 & 6.40 & 11.59 & 3.80 & 7.93 & 13.88 \\
\hline$S_{2,4}$ & 1.65 & 6.86 & 14.50 & 2.33 & 8.68 & 17.48 & 2.77 & 10.44 & 19.43 & 3.99 & 13.42 & 22.85 \\
\hline FH-Stereo ${ }_{8}$ & 1.54 & 6.22 & 11.91 & 2.22 & 7.39 & 14.64 & 2.34 & 8.51 & 15.43 & 3.14 & 10.29 & 17.96 \\
\hline$S_{2,5}$ & 1.65 & 6.95 & 12.74 & 2.23 & 8.54 & 15.64 & 2.78 & 9.58 & 17.36 & 3.30 & 11.62 & 19.46 \\
\hline FH-Stereo ${ }_{10}$ & 1.39 & 6.39 & 11.86 & 2.01 & 7.58 & 14.31 & 2.92 & 9.03 & 15.51 & 3.48 & 11.21 & 18.31 \\
\hline
\end{tabular}

Table VIII. Power of the test (\%) for the detection of omission of an interaction term between a continuous and a dichotomous covariate (Scenario 2, Table IV) averaged over all cases, broken down by number of groups $G$.

\begin{tabular}{|c|c|c|c|c|c|c|c|c|c|c|c|c|}
\hline \multirow{2}{*}{ Test } & \multicolumn{3}{|c|}{$n=100$} & \multicolumn{3}{|c|}{$n=200$} & \multicolumn{3}{|c|}{$n=400$} & \multicolumn{3}{|c|}{$n=800$} \\
\hline & $\alpha: 1 \%$ & $5 \%$ & $10 \%$ & $1 \%$ & $5 \%$ & $10 \%$ & $1 \%$ & $5 \%$ & $10 \%$ & $1 \%$ & $5 \%$ & $10 \%$ \\
\hline$S_{2,3}$ & 1.03 & 6.19 & 11.60 & 1.51 & 6.95 & 12.60 & 2.43 & 7.66 & 14.28 & 3.55 & 10.95 & 18.15 \\
\hline $\mathrm{FH}^{-S_{\text {Stereo }}}$ & 1.02 & 5.43 & 9.94 & 1.41 & 6.03 & 12.50 & 2.22 & 7.23 & 13.22 & 2.96 & 9.29 & 15.04 \\
\hline$S_{2,4}$ & 1.21 & 6.27 & 10.58 & 1.56 & 6.79 & 12.40 & 1.78 & 7.93 & 13.94 & 2.54 & 9.64 & 18.19 \\
\hline $\mathrm{FH}_{-}$Stereo $_{8}$ & 0.93 & 5.12 & 9.13 & 1.41 & 6.22 & 11.85 & 1.62 & 6.75 & 13.09 & 1.95 & 7.26 & 14.76 \\
\hline$S_{2,5}$ & 1.28 & 4.99 & 9.70 & 1.50 & 5.74 & 10.66 & 2.81 & 7.45 & 13.43 & 3.38 & 9.83 & 17.37 \\
\hline FH-Stereo ${ }_{10}$ & 0.95 & 4.71 & 9.39 & 1.19 & 5.76 & 11.74 & 1.85 & 6.89 & 12.71 & 2.57 & 8.54 & 16.33 \\
\hline
\end{tabular}

number of partition groups $G=(6,8,10)$, averaged over all cases. Similar to those for the omission of a quadratic term, the proposed test $S_{g_{1}, g_{2}}$ has higher power than the FH test for most of the cases, and the power increases as the sample size increases. Tables AX-AXII in Appendix A.2.2 give the result broken down by response categories and the distribution of the covariates.

\section{Wrong functional form of a continuous covariate}

Let the predictor in the true model contain a logarithm function of continuous covariate and a dichotomous covariate. The continuous covariate $\left(x_{1}\right)$ was simulated from a uniform distribution. Similar to the previous scenarios, the dichotomous covariate was $\left(x_{2}\right)$ generated from a Bernoulli variable. When an ordered stereotype model with the predictor $\eta_{i k}=\alpha_{k}+\phi_{k}\left(\beta_{1} x_{1}+\beta_{2} x_{2}\right)$ was fitted, Table IX shows the power to detect the wrong fitted model for both $S_{g_{1}, g_{2}}$ and FH tests using different numbers of partition groups $(G)$, averaged over various cases for the number of response categories $(q)$. Similar to the previous two scenarios, our proposed test obtains higher power than the FH test. Tables AXIII-AXV in Appendix A.2.3 show the detail results for each number of response categories. 


\begin{tabular}{|c|c|c|c|c|c|c|c|c|c|c|c|c|}
\hline \multirow{2}{*}{ Test } & \multicolumn{3}{|c|}{$n=100$} & \multicolumn{3}{|c|}{$n=200$} & \multicolumn{3}{|c|}{$n=400$} & \multicolumn{3}{|c|}{$n=800$} \\
\hline & $\alpha: 1 \%$ & $5 \%$ & $10 \%$ & $1 \%$ & $5 \%$ & $10 \%$ & $1 \%$ & $5 \%$ & $10 \%$ & $1 \%$ & $5 \%$ & $10 \%$ \\
\hline$S_{2,3}$ & 3.06 & 11.91 & 20.90 & 3.34 & 12.78 & 21.18 & 5.00 & 14.54 & 24.92 & 5.74 & 19.54 & 27.08 \\
\hline FH-Stereo 6 & 2.76 & 11.33 & 17.69 & 3.07 & 12.29 & 18.86 & 3.68 & 13.26 & 22.09 & 4.59 & 15.43 & 24.00 \\
\hline$S_{2,4}$ & 2.56 & 10.70 & 18.44 & 3.10 & 11.47 & 20.25 & 4.07 & 14.01 & 23.78 & 5.08 & 16.45 & 25.79 \\
\hline FH-Stereo ${ }_{8}$ & 2.53 & 9.49 & 14.82 & 2.76 & 10.31 & 16.99 & 3.68 & 12.19 & 19.59 & 4.06 & 12.80 & 21.77 \\
\hline$S_{2,5}$ & 2.65 & 10.88 & 17.68 & 3.34 & 11.88 & 19.31 & 4.65 & 14.30 & 22.48 & 5.62 & 16.20 & 26.20 \\
\hline $\mathrm{FH}_{-S t e r e o}{ }_{10}$ & 2.05 & 9.46 & 15.66 & 2.59 & 9.45 & 15.95 & 3.79 & 10.97 & 18.94 & 4.68 & 12.15 & 21.25 \\
\hline
\end{tabular}

Table X. Power of the test (\%) to detect the wrong ordinal response assumption (nominal response variable, Scenario 4, Table IV), averaged over all cases, broken down by number of groups $G$.

\begin{tabular}{|c|c|c|c|c|c|c|c|c|c|c|c|c|}
\hline \multirow{2}{*}{ Test } & \multicolumn{3}{|c|}{$n=100$} & \multicolumn{3}{|c|}{$n=200$} & \multicolumn{3}{|c|}{$n=400$} & \multicolumn{3}{|c|}{$n=800$} \\
\hline & $\alpha: 1 \%$ & $5 \%$ & $10 \%$ & $1 \%$ & $5 \%$ & $10 \%$ & $1 \%$ & $5 \%$ & $10 \%$ & $1 \%$ & $5 \%$ & $10 \%$ \\
\hline$S_{2,3}$ & 1.24 & 6.68 & 14.04 & 1.75 & 7.64 & 14.60 & 2.00 & 8.20 & 15.46 & 2.78 & 9.21 & 17.58 \\
\hline FH-Stereo ${ }_{6}$ & 1.66 & 7.86 & 15.05 & 2.20 & 9.05 & 17.69 & 2.93 & 10.12 & 19.98 & 3.87 & 12.34 & 20.99 \\
\hline$S_{2,4}$ & 0.96 & 5.36 & 12.53 & 1.36 & 6.93 & 13.80 & 2.01 & 7.69 & 15.48 & 2.77 & 9.61 & 17.15 \\
\hline $\mathrm{FH}-$ Stereo $_{8}$ & 1.39 & 7.16 & 14.01 & 1.75 & 8.46 & 16.09 & 2.22 & 9.85 & 18.25 & 3.24 & 11.90 & 19.83 \\
\hline$S_{2,5}$ & 1.00 & 5.60 & 11.11 & 1.29 & 6.66 & 13.08 & 1.63 & 7.22 & 14.10 & 2.11 & 8.53 & 15.79 \\
\hline $\mathrm{FH}$ Stereo $_{10}$ & 1.32 & 7.63 & 13.49 & 1.70 & 8.44 & 15.10 & 2.18 & 9.52 & 16.99 & 3.20 & 11.66 & 19.64 \\
\hline
\end{tabular}

\section{Wrong ordinal response assumption (nominal response variable)}

In this scenario, we generated data sets from a multinomial logistic model (baseline-category logit model) assuming a nominal response variable. For instance, when the number of response categories is $q=3$ with two covariates $\left(x_{1} \sim U(0,10)\right.$ or $N(5,3)$ and $\left.x_{2} \sim \operatorname{Bern}(0.5)\right)$, the predictor function has the form

$$
\eta_{i k}=\alpha_{k}+\beta_{1 k} x_{1}+\beta_{2 k} x_{2}
$$

with $\beta_{11}=0.05, \beta_{12}=0.25, \beta_{13}=0.20$ and $\beta_{2 k}=0.5$ for all $k=1,2,3$. Table AIII in Appendix A gives the parameters for each of the cases.

The fitted model was the ordered stereotype model (1) with the monotone non-decreasing constraint (2) on score parameters $\left\{\phi_{k}\right\}$. Table X shows the overall power for the proposed $S_{g_{1}, g_{2}}$ and FH tests, averaged over all cases for various partition groups $(G=6,8,10)$. Unlike the previous scenarios, the $\mathrm{FH}$ test is slightly better than our proposed test for this scenario. Because the response categories do not have nature ordering, the score parameters $\left\{\phi_{k}\right\}$ in the ordered stereotype model (1) with the monotone nondecreasing constraint (2) are meaningless. Therefore, the weighted score (4) calculated based on $\left\{\hat{\phi}_{k}\right\}$ does not provide any advantage on the group partitioning. Tables AXVI-AXVIII in Appendix A.2.4 show the results separated for each case.

\section{Omission of an interaction term between two dichotomous covariates}

The true model includes the interaction term between two dichotomous covariates. It also considers the main effects of an independent continuous covariate $(\mathcal{N}(5,3))$. We simulated the dichotomous covariates as Bernoulli variables with the probability of success of $0.2,0.5$, and 0.7 . The power of the test depends on several things such as the type of model, if the responses are balanced or not, and the values of the coefficients. We intentionally chose the true value of the coefficients $\left\{\beta_{j}\right\}$ to be larger than those from other scenarios (Tables IV and AII in Appendix A) to observe that indeed the power values are larger in this scenario. Table XI shows the power to detect the missing interaction term for each number of partition groups $G=(6,8,10)$, averaged over all cases. The proposed test $S_{g_{1}, g_{2}}$ has very satisfactory results and higher power than the FH test for all the cases. The power increases as the sample size increases, with a highest value of $82.47 \%$. Tables AXIX-AXXI in Appendix A.2.5 give the result broken down by response categories and the distribution of the covariates. 


\begin{tabular}{|c|c|c|c|c|c|c|c|c|c|c|c|c|}
\hline \multirow{2}{*}{ Test } & \multicolumn{3}{|c|}{$n=100$} & \multicolumn{3}{|c|}{$n=200$} & \multicolumn{3}{|c|}{$n=400$} & \multicolumn{3}{|c|}{$n=800$} \\
\hline & $\alpha: 1 \%$ & $5 \%$ & $10 \%$ & $1 \%$ & $5 \%$ & $10 \%$ & $1 \%$ & $5 \%$ & $10 \%$ & $1 \%$ & $5 \%$ & $10 \%$ \\
\hline$S_{2,3}$ & 2.98 & 13.41 & 21.36 & 9.24 & 33.92 & 40.9 & 30.31 & 56.58 & 65.51 & 40.25 & 67.34 & 77.72 \\
\hline FH-Stereo $_{6}$ & 1.42 & 8.31 & 15.07 & 3.14 & 13.37 & 20.16 & 12.04 & 28.13 & 29.33 & 19.21 & 38.23 & 57.38 \\
\hline $\begin{array}{l}S_{2,4} \\
\text { FH-Stereo }\end{array}$ & $\begin{array}{l}3.56 \\
1.45\end{array}$ & $\begin{array}{c}11.38 \\
7.70\end{array}$ & $\begin{array}{l}21.44 \\
13.94\end{array}$ & $\begin{array}{l}11.29 \\
3.05\end{array}$ & $\begin{array}{l}32.12 \\
12.11\end{array}$ & $\begin{array}{l}38.91 \\
18.70\end{array}$ & $\begin{array}{l}39.75 \\
10.74\end{array}$ & $\begin{array}{l}53.58 \\
27.66\end{array}$ & $\begin{array}{l}63.79 \\
24.59\end{array}$ & $\begin{array}{l}46.19 \\
17.51\end{array}$ & $\begin{array}{l}59.60 \\
36.18\end{array}$ & $\begin{array}{l}82.47 \\
53.09\end{array}$ \\
\hline & 2.8 & 11.93 & 21.47 & 869 & 35.63 & 42.3 & 292 & 60.04 & 673 & 33.28 & 64.31 & 77.44 \\
\hline FH-Stereo ${ }_{10}$ & 1.21 & 7.08 & 14.04 & 2.55 & 11.18 & 19.14 & 8.94 & 25.35 & 25.12 & 14.35 & 33.14 & 53.53 \\
\hline
\end{tabular}

Table XII. Power of the test (\%) for the detection of omission of a continuous covariates which has not interacted with the other covariates in the model (Scenario 6, Table IV) averaged over all cases, broken down by number of groups $G$.

\begin{tabular}{|c|c|c|c|c|c|c|c|c|c|c|c|c|}
\hline \multirow{2}{*}{ Test } & \multicolumn{3}{|c|}{$n=100$} & \multicolumn{3}{|c|}{$n=200$} & \multicolumn{3}{|c|}{$n=400$} & \multicolumn{3}{|c|}{$n=800$} \\
\hline & $\alpha: 1 \%$ & $5 \%$ & $10 \%$ & $1 \%$ & $5 \%$ & $10 \%$ & $1 \%$ & $5 \%$ & $10 \%$ & $1 \%$ & $5 \%$ & $10 \%$ \\
\hline$S_{2,3}$ & 6.35 & 17.17 & 46.81 & 7.05 & 43.75 & 53.25 & 25.66 & 58.72 & 62.76 & 28.22 & 60.57 & 67.91 \\
\hline FH-Stereo 6 & 6.14 & 15.24 & 33.71 & 6.49 & 30.97 & 34.67 & 13.41 & 31.82 & 37.48 & 14.09 & 36.29 & 38.13 \\
\hline & 7.13 & 20.51 & 43.86 & 7.92 & 52.29 & 49.92 & 28.85 & 70.20 & 58.85 & 31.75 & 72.45 & 63.71 \\
\hline FH-Stereo 8 & 6.63 & 15.04 & 31.05 & 6.99 & 30.54 & 31.92 & 14.47 & 31.38 & 34.54 & 15.19 & 35.83 & 35.20 \\
\hline & 6.88 & 19.83 & 46.68 & 7.62 & 51.43 & 53.11 & 27.76 & 68.39 & 62.63 & 30.57 & 70.68 & 67.78 \\
\hline $\mathrm{FH}-$ Stereo $_{10}$ & 6.66 & 17.08 & 29.89 & 6.99 & 34.76 & 30.74 & 14.48 & 35.73 & 33.29 & 15.21 & 40.78 & 33.93 \\
\hline
\end{tabular}

\section{Omission of a continuous covariate (independent of the other covariates)}

In this scenario, the model considered has three continuous covariates (two $\mathcal{N}(5,3)$ variables and a $\mathcal{U}(0,10)$ variable) and no interaction term between them. We have explored the omission of one of the continuous covariates $(\mathcal{U}(0,10))$. Table XII shows that the power of the proposed test $S_{g_{1}, g_{2}}$ is twice the power for most of the cases compared with the FH test. The full results are given in Tables AXXII-AXXIV in Appendix A.2.6.

\section{Omission of an interaction term when all the variables in the model are dichotomous}

The true model includes the interaction term between two dichotomous covariates in a model along with their main effects. We simulated covariates from Bernoulli variables with the probability of success of 0.2 , 0.5 , and 0.7 . Table XIII shows the power to detect the missing interaction term for each number of partition groups $G=(6,8,10)$, averaged over all cases. The proposed test $S_{g_{1}, g_{2}}$ has very satisfactory results; on average, it doubled the results of the FH test. Tables AXXV-AXXVII in Appendix A.2.7 give the result broken down by response categories and the distribution of the covariates. However, we are careful in our conclusions of the results for this scenario. This scenario is configured with only four covariate patterns with unbalanced sizes; therefore, it may not result in reasonable groups using the proposed or FH method. The estimated probabilities $\hat{\theta}_{i k}$ (Eq. (3)) for each observation $i=1, \ldots, n$ and response category $k=1, \ldots, q$ have only four distinct sets corresponding to four covariate patterns, respectively. Therefore, there are only four distinct weighted scores. If we divide them into $G$ groups that contain an equal number of observations, the lack of fit information might be missed due to the amalgamation. It is not surprising because the approximate chi-square distribution of the traditional Hosmer-Lemeshow test works well when the number of distinct covariate patterns equals the sample size [10]. In this case, our recommendation is to use the classical Pearson $\chi^{2}$ test if there are few covariate patterns.

\section{Rejection of the null distribution and power of the test with non-equal spacing}

In order to compare the proposed test with the FH test [15], all the scenarios tested in the simulation study were formulated with equal spacing among ordinal response categories as the FH test statistic is calculated using this assumption to find partitioning groups. However, our proposed test is not taking the full advantage of the use of the fitted spacing under these scenarios. Based on the proposed partitioning 


\begin{tabular}{|c|c|c|c|c|c|c|c|c|c|c|c|c|}
\hline \multirow{2}{*}{ Test } & \multicolumn{3}{|c|}{$n=100$} & \multicolumn{3}{|c|}{$n=200$} & \multicolumn{3}{|c|}{$n=400$} & \multicolumn{3}{|c|}{$n=800$} \\
\hline & $\alpha: 1 \%$ & $5 \%$ & $10 \%$ & $1 \%$ & $5 \%$ & $10 \%$ & $1 \%$ & $5 \%$ & $10 \%$ & $1 \%$ & $5 \%$ & $10 \%$ \\
\hline$S_{2,3}$ & 2.78 & 12.73 & 22.89 & 3.13 & 13.08 & 23.63 & 3.48 & 13.39 & 23.86 & 4.10 & 14.88 & 25.65 \\
\hline FH-Stereo $_{6}$ & 0.73 & 4.13 & 8.89 & 1.32 & 4.41 & 9.61 & 1.52 & 5.18 & 9.91 & 1.81 & 5.42 & 10.03 \\
\hline$S_{2,4}$ & 2.64 & 11.26 & 19.72 & 2.99 & 11.66 & 20.41 & 3.34 & 11.97 & 20.68 & 3.97 & 13.26 & 22.15 \\
\hline FH-Stereo ${ }_{8}$ & 0.71 & 4.09 & 9.18 & 1.30 & 4.38 & 9.92 & 1.51 & 5.18 & 10.27 & 1.83 & 5.45 & 10.44 \\
\hline$S_{2,5}$ & 3.47 & 13.16 & 23.69 & 3.91 & 13.55 & 24.49 & 4.32 & 13.89 & 24.75 & 5.12 & 15.47 & 26.63 \\
\hline $\mathrm{FH}$ Stereo $_{10}$ & 0.77 & 4.22 & 8.71 & 1.42 & 4.52 & 9.43 & 1.64 & 5.35 & 9.77 & 1.98 & 5.65 & 9.93 \\
\hline
\end{tabular}

\begin{tabular}{|c|c|c|c|c|c|c|c|c|c|c|c|c|c|c|}
\hline \multirow{2}{*}{ Test } & \multirow{2}{*}{ Covariates } & \multirow{2}{*}{ Rates } & \multicolumn{3}{|c|}{$n=100$} & \multicolumn{3}{|c|}{$n=200$} & \multicolumn{3}{|c|}{$n=400$} & \multicolumn{3}{|c|}{$n=800$} \\
\hline & & & $1 \%$ & $5 \%$ & $10 \%$ & $1 \%$ & $5 \%$ & $10 \%$ & $1 \%$ & $5 \%$ & $10 \%$ & $1 \%$ & $5 \%$ & $10 \%$ \\
\hline \multirow{2}{*}{$\begin{array}{l}S_{2,3} \\
\mathrm{G}=6\end{array}$} & \multirow{2}{*}{$\mathcal{N}(5,3)$} & Reject & 2.01 & 8.49 & 12.79 & 1.81 & 6.04 & 11.11 & 1.49 & 5.52 & 10.34 & 1.03 & 5.19 & 10.19 \\
\hline & & Power & 2.53 & 7.48 & 13.89 & 3.11 & 9.79 & 17.78 & 4.41 & 12.29 & 20.37 & 5.46 & 15.11 & 27.17 \\
\hline \multirow{2}{*}{$\begin{array}{l}S_{2,4} \\
\mathrm{G}=8\end{array}$} & \multirow{2}{*}{$\mathcal{U}(0,10)$} & Reject & 3.76 & 7.02 & 12.54 & 2.09 & 6.76 & 11.93 & 1.39 & 6.19 & 10.61 & 1.19 & 5.35 & 10.19 \\
\hline & & Power & 3.55 & 15.12 & 22.61 & 5.97 & 18.46 & 29.82 & 6.05 & 21.41 & 34.07 & 7.41 & 24.56 & 34.19 \\
\hline \multirow{2}{*}{$\begin{array}{l}S_{2,5} \\
\mathrm{G}=10\end{array}$} & $\mathcal{U}(0,10)$ and & Reject & 2.21 & 9.07 & 13.64 & 1.44 & 5.79 & 12.16 & 1.37 & 5.68 & 11.07 & 1.14 & 5.44 & 10.08 \\
\hline & $\operatorname{Bern}(0.5)$ & Power & 3.89 & 8.27 & 9.66 & 4.73 & 11.49 & 12.99 & 5.44 & 12.84 & 17.88 & 5.56 & 13.15 & 21.95 \\
\hline
\end{tabular}

Note: $S_{g_{1}, g_{2}}$ is the proposed test with the first partition (deviance) into $g_{1}$ and the second partition into $g_{2}$ groups; and $G=g_{1} \times g_{2}$.

scheme in Section 3, we expect that the performance of the $S_{g_{1}, g_{2}}$ test will improve in the case of uneven spacing. Thus, we have tested a subset of the scenarios modifying the score parameters to introduce nonequal spacing (e.g., $\phi=(0,0.2,0.7,1)$, when the number of response categories is $q=4)$. Table XIV shows the results of the $S_{g_{1}, g_{2}}$ test for rejection rates of the null distribution and for the power of the test to detect an omitted quadratic term in a continuous covariate (Scenario 1, Table IV), which has greater power than those from equal spacing (Table VII).

\section{Applications}

In this section, we illustrate the application of the proposed $S_{g_{1}, g_{2}}$ test with two real-life examples that come from the study reported by Fontanella et al. [22]. The study collected medical records of 508 adolescents admitted to a psychiatric hospital, to find factors that influence aftercare placement for hospitalized adolescents. The data contain 14 variables about sociodemographic, clinical, treatment and family characteristics, and service history. Several authors $[9,15]$ used this dataset to assess the performance of goodness-of-fit for ordinal models, without aiming to give a complete assessment of the factors. Similarly, we do not focus on examining comprehensively the main factors that influence aftercare placement. We fit an ordered stereotype model for these data to illustrate the proposed goodness-of-fit test and compare it with the test of the proportional odds model given by Fagerland and Hosmer [15]. In order to compare the tests, the ordered stereotype model has the same set of covariates as the proportional odds model given by Fagerland and Hosmer [15]. The covariates include dichotomous and continuous variables similar to the configures scenarios in the simulation study (Section 4).

\subsection{Example 1: Neuropsychiatric disturbance in aftercare placement}

In this first example, the ordinal response variable was the neuropsychiatric disturbance (Neuro) for $n=508$ adolescents. The covariates included in the analysis were age, age $^{2}$, gender, race, emotional disturbance and state custody. Table XV describes all the variables and their possible values. 


\begin{tabular}{|c|c|c|c|}
\hline Type & Variable & Description & Values \\
\hline Response & Neuro & Neuropsychiatric disturbance & $\begin{array}{l}1=\text { none } \\
2=\text { mild } \\
3=\text { moderate } \\
4=\text { severe }\end{array}$ \\
\hline \multirow{5}{*}{ Covariates } & Gender & Gender of the adolescent & $\begin{array}{l}0=\text { female } \\
1=\text { male }\end{array}$ \\
\hline & Race & Race of the adolescent & $\begin{array}{l}0=\text { white } \\
1=\text { non }- \text { white }\end{array}$ \\
\hline & Emot & Emotional disturbance & $\begin{array}{l}0=\text { mild } \\
1=\text { severe }\end{array}$ \\
\hline & Cust & State custody & $\begin{array}{l}0=\text { no } \\
1=\text { yes }\end{array}$ \\
\hline & $\begin{array}{l}\text { Age } \\
\mathrm{Age}^{2}\end{array}$ & \multicolumn{2}{|c|}{$\begin{array}{l}\text { Age in years, centered about the mean age } \\
\text { Age }^{2} \text { in years, centered about the mean age }\end{array}$} \\
\hline
\end{tabular}

\begin{tabular}{|c|c|c|c|c|}
\hline Coefficient & Estimation & S.E. & $P$-value & 95\% C.I. \\
\hline$\widehat{\alpha}_{2}$ & -1.992 & 0.235 & & $(-2.452,-1.531)$ \\
\hline$\widehat{\alpha}_{3}$ & -3.019 & 0.278 & & $(-3.564,-2.474)$ \\
\hline$\widehat{\alpha}_{4}$ & -2.516 & 0.252 & & $(-3.010,-2.021)$ \\
\hline$\widehat{\beta_{1}}$ (Gender) & 0.293 & 0.199 & 0.141 & $(-0.099,0.685)$ \\
\hline$\widehat{\beta}_{2}($ Race $)$ & 0.382 & 0.200 & 0.056 & $(-0.010,0.773)$ \\
\hline$\widehat{\beta}_{3}($ Emot $)$ & 0.627 & 0.231 & 0.007 & $(0.174,1.080)$ \\
\hline$\widehat{\beta}_{4}($ Cust $)$ & -0.487 & 0.210 & 0.020 & $(-0.899,-0.074)$ \\
\hline$\hat{\beta}_{5}($ Age $)$ & -0.008 & 0.058 & 0.890 & $(-0.122,0.107)$ \\
\hline$\hat{\beta}_{6}\left(\operatorname{Age}^{2}\right)$ & 0.065 & 0.031 & 0.036 & $(0.004,0.126)$ \\
\hline$\hat{\phi}_{2}$ & 0.406 & 0.114 & & $(0.183,0.630)$ \\
\hline$\widehat{\phi}_{3}$ & 0.898 & 0.121 & & $(0.661,1.000)$ \\
\hline
\end{tabular}

Table XVI gives the ordered stereotype model fitting result showing that the covariates age ${ }^{2}$, emotional disturbance (Emot), and state custody (Cust) have a significant effect on the neuropsychiatric disturbance. We obtained very similar significance results for the effect of covariates $\left\{\beta_{j}\right\}$ as the proportional odds model given by Fagerland and Hosmer [15, Table IX]. The fitted scores parameters $\left\{\phi_{k}\right\}$ determine the spacing between adjacent ordinal categories, which is an advantage as a result of fitting an ordered stereotype model. In this first example, the fitted score shows uneven spacing $\left(\left\{\hat{\phi}_{k}\right\}=(0,0.41,0.90,1)\right)$.

Several goodness-of-fit tests of the proportional odds model were conducted by FH [15], including the $\mathrm{FH}$ test $\left(\mathrm{FH}_{10}\right.$, using $G=10$ partition groups), the Lipsitz test (Lipsitz), the modified Pearson $\left(\operatorname{PR}\left(\chi^{2}\right)\right)$, and the deviance $\left(\operatorname{PR}\left(D^{2}\right)\right)$ of the Pulkstenis and Robinson tests. For the ordered stereotype model, we calculated both the proposed test $S_{g_{1}, g_{2}}$ and the FH test, where the FH test is based on the fitted probabilities from the ordered stereotype model, denoted by FH-Stereo using two grouping options. One forced the tests to have $G=10$ groups in order to make the results comparable with those from FH [15]. The other imposed the requirement that all the expected frequencies should be greater than 1 and at least $80 \%$ should be greater than 5 for a good $\chi^{2}$-approximation. The latter option resulted in $G=6$ groups as the best grouping setting for our proposed test $S_{2,3}$ and $G=7$ groups for the FH-stereo test. Table XVII shows $p$-values for all the tests. All the test results for both models show no evidence of lack of fit (e.g., $p$-value $=0.48$ for the $S_{2,3}$ test). In Appendix B.1, Figure B1 depicts the test statistic value under the null 


\begin{tabular}{|lcc|}
\hline \multicolumn{3}{|l|}{ Table XVII. Neuropsychiatric disturbance in } \\
aftercare placement data set: $P$-values of the \\
goodness-of-fit tests for the proportional odds \\
model or the ordered stereotype model. \\
Null distribution & Test & $P$-value \\
\hline & $\mathrm{FH}_{10}$ & 0.73 \\
Proportional odds model & $\operatorname{Lipsitz}$ & 0.45 \\
& $\operatorname{PR}\left(\chi^{2}\right)$ & 0.62 \\
& $\operatorname{PR}\left(D^{2}\right)$ & 0.39 \\
& $\mathrm{FH}-$ Stereo $_{10}$ & 0.67 \\
Ordinal stereotype model & $S_{2,5}$ & 0.32 \\
\cline { 2 - 3 } & $\mathbf{F H - S t e r e 0 _ { 7 }}$ & $\mathbf{0 . 1 4}$ \\
& $\boldsymbol{S}_{\mathbf{2 , 3}}$ & $\mathbf{0 . 4 8}$ \\
\hline
\end{tabular}

Note: Tests include the Fagerland-Hosmer with $G=10$ groups $\left(\mathrm{FH}_{10}\right)$, the Lipsitz test, the modified Pearson $\left(\operatorname{PR}\left(\chi^{2}\right)\right)$ and the deviance $\left(\operatorname{PR}\left(D^{2}\right)\right)$ of the Pulkstenis and Robinson tests, the Fagerland-Hosmer test with the fitted probabilities from the ordinal stereotype model and $G$ groups (FH-Stereo ${ }_{G}$ ), and the proposed test with $g_{1}$ (first) and $g_{2}$ (second) grouping $\left(S_{g_{1}, g_{2}}\right)$.

hypothesis that the ordered stereotype model fits the data, and Table BI shows the table of contingency of observed and expected frequencies following the fit in Table XVI.

\subsection{Example 2: Dangers to others in aftercare placement}

The second example uses a different subset of variables from the data set of medical records of 508 adolescents admitted to a psychiatric hospital. In this case, the response variable is an assessment of the risk of danger to others associated each each patient (Danger) which has four ordinal levels: $1=$ unlikely, $2=$ possibly, $3=$ probably, and $4=$ likely. The covariates included to fit the stereotype model and the proportional odds model were age, gender, number of days staying in the hospital (LOS), behavioral symptom (Behave), and elopement risk (Elope). Table BII in Appendix B.2 describes all the variables and their possible values. Additionally, Table BIII gives the model fitting results showing that the covariates of gender, elopement risk, and behavioral symptom are significant for the dangers the patients pose to others. The estimated score parameters $\left(\left\{\phi_{k}\right\}=(0,0.65,0.85,1)\right)$ indicate uneven spacing among ordinal response levels.

Table XVIII shows $p$-values of various goodness-of-fit tests for the proportional odds and ordered stereotype models as mentioned in the first example. For the proportional odds model, the FH and Pulkstenis and Robinson tests show the evidence of lack of fit at a 5\% of significance level, but the Lipsitz test does not show the lack of fit. Fagerland and Hosmer [15] suggested that this divergence could be explained as a result that the assumption of proportion odds does not seem to be satisfied for the fitted model.

For the ordered stereotype model, the $G=10$ group partition satisfies the requirement that all the expected frequencies should be greater than 1 and at least $80 \%$ should be greater than 5 . However, the results of the $\mathrm{FH}$ test $\left(\mathrm{FH}-\mathrm{Stereo}_{10}\right)$ and the proposed $S_{2,5}$ test are different. Unlike the proposed test with the $p$-value of 0.26 , the FH test suggests a lack of fit at a $5 \%$ of significance level ( $p$-value $=0.0003$ ). Table BIV in Appendix B.2 gives a $10 \times 4(G \times q)$ table of observed and expected frequencies by crossclassifying the response levels and the partitioning groups according to the scheme in Section 3. It shows little evidence of lack of fit that supports the result of the proposed test. The ordered stereotype model which does not assume the proportional odds assumptions seems to have a better fit than the proportional odds model. Because the estimated level of spacing between response categories is far away from the equal spacing for this example, FH's partitioning scheme results in quite different groups from the proposed method. It also suggests that the Hosmer-Lemeshow test [10] is sensitive to the grouping method in general. 


\begin{tabular}{|c|c|c|}
\hline \multicolumn{3}{|c|}{$\begin{array}{l}\text { Table XVIII. Dangers to others in aftercare place- } \\
\text { ment data set: } P \text {-values of the goodness-of-fit tests } \\
\text { for the proportional odds model or the ordered } \\
\text { stereotype model. }\end{array}$} \\
\hline Null distribution & Test & $P$-value \\
\hline Proportional odds model & $\begin{array}{l}\mathrm{FH}_{10} \\
\mathrm{Lipsitz} \\
\operatorname{PR}\left(\chi^{2}\right) \\
\operatorname{PR}\left(D^{2}\right)\end{array}$ & $\begin{array}{c}0.0004 \\
0.54 \\
0.0051 \\
0.0056\end{array}$ \\
\hline Ordered stereotype model & $\begin{array}{c}\text { FH-Stereo } \\
S_{2,5} \\
\end{array}$ & $\begin{array}{c}0.0003 \\
0.26\end{array}$ \\
\hline
\end{tabular}

Note: Tests include the Fagerland-Hosmer with $G=$ 10 groups $\left(\mathrm{FH}_{10}\right)$, the Lipsitz test, the modified Pearson $\left(\operatorname{PR}\left(\chi^{2}\right)\right)$ and the deviance $\left(\operatorname{PR}\left(D^{2}\right)\right)$ of the Pulkstenis and Robinson tests, the Fagerland-Hosmer test with the fitted probabilities from the ordinal stereotype model and $G$ groups $\left(\mathrm{FH}-\right.$ Stereo $\left._{G}\right)$, and the proposed test with $g_{1}$ (first) and $g_{2}$ (second) grouping $\left(S_{g_{1}, g_{2}}\right)$.

\section{Discussion}

This paper introduces a new goodness-of-fit test for ordered stereotype models based on the HosmerLemeshow test for logistic regression and its version for the proportional odds model. The latter test is calculated based on a grouping scheme assuming that the levels of the ordinal response are equal spaced which, based on our experience, might be not true. Because the ordered stereotype model allows us to determine a new uneven spacing of the ordinal categories, dictated by the data, the grouping scheme can be improved by using the spacing information. This new adjusted spacing is found by the estimated score parameters $\left\{\widehat{\phi}_{k}\right\}$, and they are incorporated in the calculation of the proposed $S_{g_{1}, g_{2}}$ test. It provides more accurate information on the weighted scores to partition data into groups. As the derivation of the proposed test follows closely the well known Hosmer-Lemeshow test, its use and interpretation will be familiar to applied researchers.

We have demonstrated the performance of the proposed test by means of two examples with real data and have tested its reliability by setting up a simulation study in a diverse range of scenarios. We assessed both the null distribution and the power of the test. The $S_{g_{1}, g_{2}}$ test performed best in almost all the cases. However, there are some limitations of the test, including the choice of $g_{1}$ and the number of distinct covariate patterns. The aim of introducing an additional partition based on the deviances is to pick up some important deviations because of a small number of observations which may be missed by grouping with the weighted scores only. When $g_{1}$ is too big, it partitions the lowest and highest deviations extremely and leads to a large value of the test statistics, even if the null model holds. It might incorrectly signal a lack of fit. We did a small number of simulations for $g_{1}=3$ and $g_{1}=4$ that showed an inflated nominal significance level. We recommend to use $g_{1}=2$. When the number of distinct covariate patterns is small, using the same number of observations in each partitioning group might result in inappropriate groups. If there is no continuous covariate, one is better to use the classical Pearson $\chi^{2}$ or the deviance statistic.

Computation can be slow in this stage, although we have substantially reduced the time required by calling $\mathbf{C}$ from $\mathbf{R}$. There is no clear working rule for determining $g_{2}$ that partitions the observations based on the weighted scores. The simulation study shows that the performance of the test is robust to different number of groups $g_{2}$, although the power seems to slightly higher when the number of groups is smaller. It is not surprising, because for a chi-squared test of independence in a contingency table the power increases as degrees of freedom decreases under a fixed non-centrality parameter [23]. In any case, we recommend to impose the requirement that all the calculated expected frequencies should be greater than 1 and at least $80 \%$ should be greater than 5 for the $\chi^{2}$-approximation to hold. One possible strategy would be to follow the technique of the deciles of risk (i.e., use 10 groups) which is the default in most of software packages for the binary case of the Hosmer-Lemeshow test. Thus, we start creating $G=2 \times 5=10$ groups using the proposed partitioning scheme to assess whether the sample size requirement holds for the cell expected frequencies in the contingency table. If not, iteratively reduce the number of partitioning groups until the requirement is accomplished. 
We observe that the performance of the proposed test is better than the FM test for the equal spacing scenarios (Tables III and V). Rejection rates of the null distribution are closer to the nominal significance level, and the testing power is also higher. We also observe that the results were even better in the case of uneven spacing (Table XIV).

An important future development would be to develop a goodness-of-fit test when data consist of $m$ ordinal response variables each with $q$ categories measured on a set of $n$ units. In this case, the data can be represented by an $n \times m$ matrix where, for instance, the $n$ rows represent the subjects of the study and the $m$ columns are the different questions in a particular questionnaire. Note that the data do not include covariates and the structure of the linear predictor in the ordered stereotype model may simply be related to the effect of the row and column on each observation. Thus, a possible formulation would be as follows:

$$
\log \left(\frac{P\left[Y_{i j}=k\right]}{P\left[Y_{i j}=1\right]}\right)=\alpha_{k}+\phi_{k}\left(\tau_{i}+\beta_{j}\right), \quad k=2, \ldots, q, \quad i=1, \ldots, n, \quad j=1, \ldots, m,
$$

where we define $\left\{\tau_{1}, \ldots, \tau_{n}\right\}$ and $\left\{\beta_{1}, \ldots, \beta_{m}\right\}$ as the sets of parameters quantifying the main effects of the $n$ rows and $m$ columns, respectively. The main problem of the parametrization in this model is that the specific row and column effects could over-parametrize the data structure. This new goodness-of-fit must take into account the possible clustering structure to reducing the dimensionality of the problem and become a parsimonious model (see Fernádez et al. [20] for more discussion about it).

Another research direction to explore would be to generalize alternative grouping methods used for the binary case [24-28] and compare them. In this paper, the optim() algorithm was repeated 10 times with random starting points, and the best ML estimates were kept. Another approaches to determine the starting points must be analyzed and compare their performances. Finally, the ordered stereotype model that describes all predictors using the same scores $\left\{\phi_{k}\right\}$ might not be true. A more flexible stereotype model allows different scores for different predictors. Thus, the weighted score in the proposed grouping scheme needs to be modified according to the predictors.

\section{Appendix A: Results simulation study}

TA1 - TA2

TA4 TA6

TA7 - TA9

TA10 TA12

TA13 - TA15

$\ddot{\theta}$

All the parameters for each scenario in the simulation study for assessing the power of the test are shown in Tables AI (Scenarios 1-4) and AII (Scenarios 5-7).

\section{A.1. Assessing the rejection of the null distribution}

Tables AIV-AVI show proportions of times that the test statistic exceeds the $100(1-\alpha)$ percentage point of the null chi-squared distribution when the fitted model is the true model (1) with $q=3,4,5$, respectively.

\section{A.2. Assessing the power test}

A.2.1. Omission of a quadratic term in a continuous covariate. Tables AVII-AIX present proportions of times that the null hypothesis was rejected at a certain fixed level of significance, over 10,000 simulated data sets for each test statistic when the data were simulated from Scenario 1 in Table IV with the response category of $q=3,4,5$, respectively.

A.2.2. Omission of an interaction term between a continuous and a dichotomous covariate. Tables AXAXII give power to detect the missing interaction term between a continuous and a dichotomous covariate when the data were simulated from Scenario 2 in Table IV with the response category of $q=3,4,5$, respectively.

A.2.3. Wrong functional form of a continuous covariate. Tables AXIII-AXV have the detailed power to detect the wrong functional form of a continuous covariate when the data were simulated from the model that contains a logarithm function of continuous covariate from Scenario 3 in Table IV with the response category of $q=3,4,5$, respectively. 


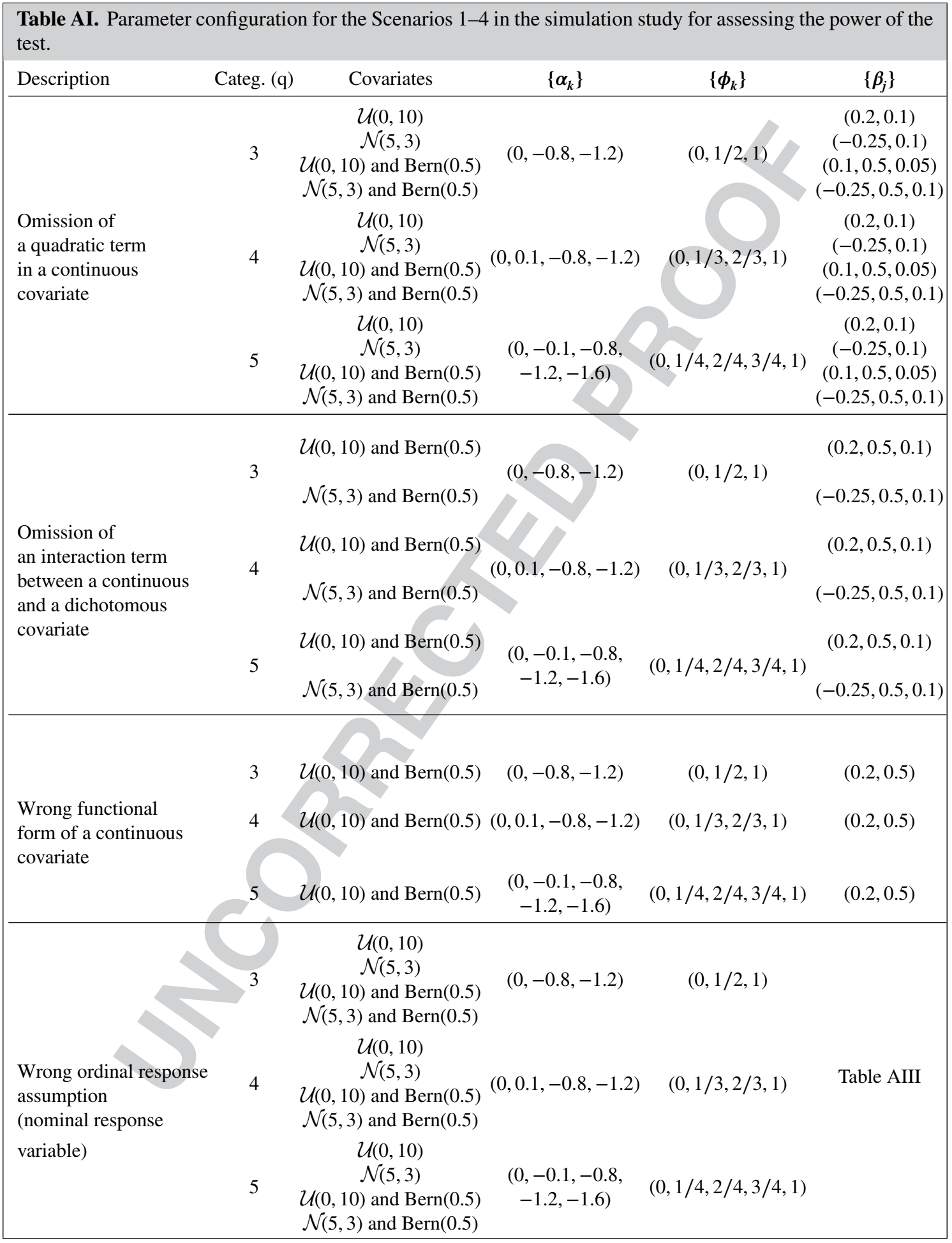




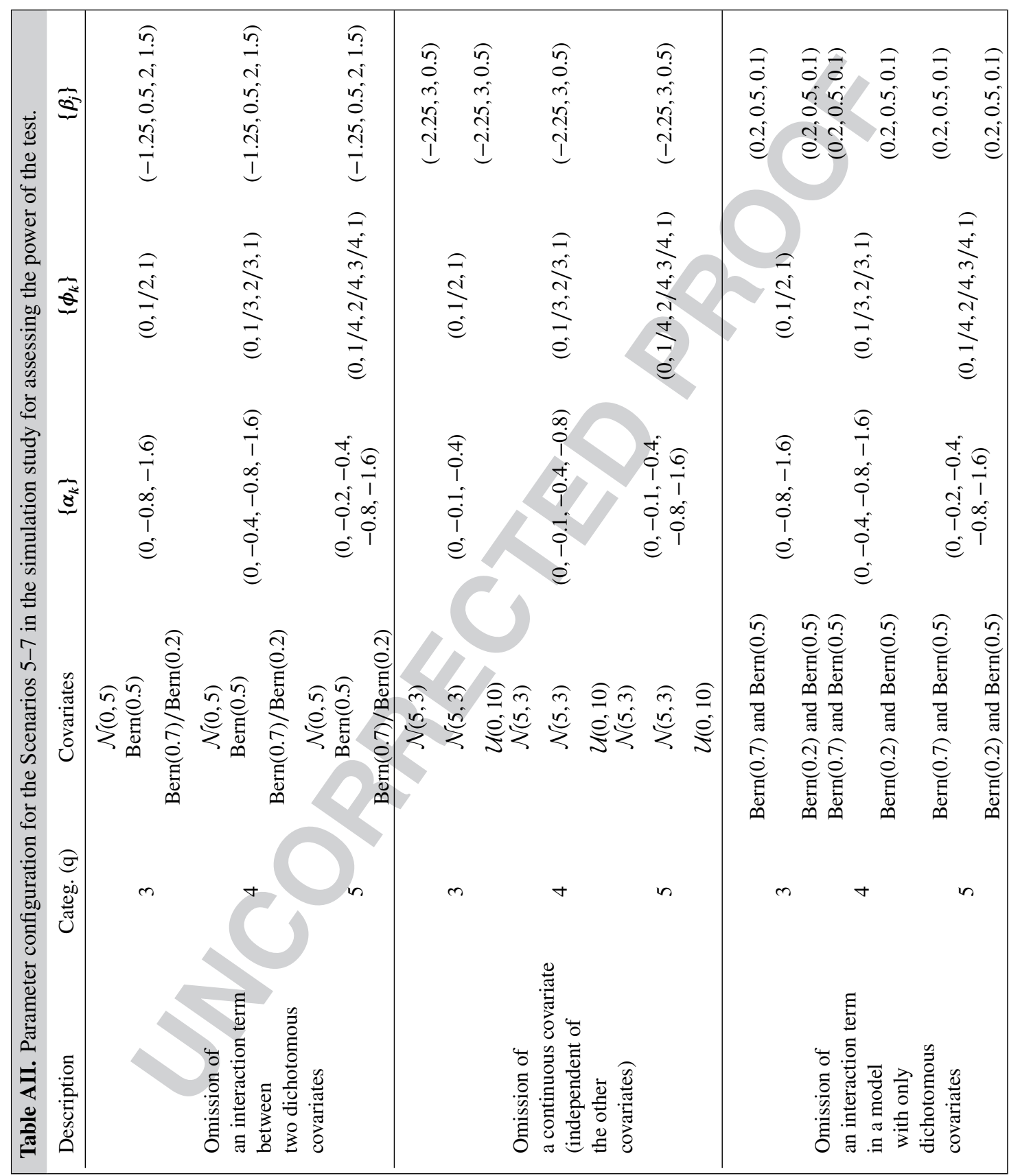




\begin{tabular}{|c|c|c|c|}
\hline Description & Categ. (q) & Covariates & $\beta^{T}$ \\
\hline \multirow{4}{*}{$\begin{array}{l}\text { Wrong ordinal response } \\
\text { assumption } \\
\text { (nominal response } \\
\text { variable) }\end{array}$} & \multirow{2}{*}{3} & $\begin{array}{c}\mathcal{U}(0,10) \\
\mathcal{N}(5,3)\end{array}$ & $\left\{\beta_{k}\right\}=\{0.05,0.25,0.20\}$ \\
\hline & & $\begin{array}{c}\mathcal{U}(0,10) \text { and } \operatorname{Bern}(0.5) \\
\mathcal{N}(5,3) \text { and } \operatorname{Bern}(0.5)\end{array}$ & $\begin{array}{l}\left\{\beta_{1 k}\right\}=\{0.05,0.25,0.20\} \\
\left\{\beta_{2 k}\right\}=\{0.50,0.50,0.50\}\end{array}$ \\
\hline & 4 & $\begin{array}{c}\mathcal{U}(0,10) \\
\mathcal{N}(5,3) \\
\mathcal{U}(0,10) \text { and } \operatorname{Bern}(0.5) \\
\mathcal{N}(5,3) \text { and } \operatorname{Bern}(0.5)\end{array}$ & $\begin{array}{l}\left\{\beta_{k}\right\}=\{0.20,0.15,0.25,0.35\} \\
\left\{\beta_{1 k}\right\}=\{0.20,0.15,0.25,0.35\} \\
\left\{\beta_{2 k}\right\}=\{0.50,0.50,0.50,0.50\}\end{array}$ \\
\hline & 5 & $\begin{array}{c}\mathcal{U}(0,10) \\
\mathcal{N}(5,3) \\
\mathcal{U}(0,10) \text { and } \operatorname{Bern}(0.5) \\
\mathcal{N}(5,3) \text { and } \operatorname{Bern}(0.5)\end{array}$ & $\begin{array}{l}\left\{\beta_{k}\right\}=\{0.15,0.10,0.35,0.25,0.38\} \\
\left\{\beta_{1 k}\right\}=\{0.15,0.10,0.35,0.25,0.38\} \\
\left\{\beta_{2 k}\right\}=\{0.50,0.50,0.50,0.50,0.50\}\end{array}$ \\
\hline
\end{tabular}

Table AIV. Proportion of times (\%) that the test statistic exceeds the $100(1-\alpha)$ percentage point of the null chi-squared distribution when the fitted model is the true model (1) with $q=3$ ordinal categories.

\begin{tabular}{|c|c|c|c|c|c|c|c|c|c|c|c|c|c|}
\hline \multirow{2}{*}{ Test } & \multirow{2}{*}{ ovariates } & \multicolumn{3}{|c|}{$n=100$} & \multicolumn{3}{|c|}{$n=200$} & \multicolumn{3}{|c|}{$n=400$} & \multicolumn{3}{|c|}{$n=800$} \\
\hline & & $\alpha: 1 \%$ & $5 \%$ & $10 \%$ & $1 \%$ & $5 \%$ & $10 \%$ & $1 \%$ & $5 \%$ & $10 \%$ & $1 \%$ & $5 \%$ & $10 \%$ \\
\hline$S_{2,3}$ & & 3.84 & 7.06 & 12.08 & 2.18 & 6.08 & 11.52 & 1.91 & 5.96 & 11.41 & 1.85 & 5.96 & 10.84 \\
\hline FH-Stereo 6 & & 3.14 & 6.91 & 12.97 & 3.05 & 6.21 & 81 & 65 & 5.46 & 11.77 & 1.97 & 26 & 10.82 \\
\hline$S_{2,4}$ & & 3.46 & 8.45 & 12.41 & 3.22 & 7.69 & 11.25 & 1.99 & 6.36 & 11.01 & 1.69 & 5.57 & 10.71 \\
\hline FH-Stereo 8 & & 3.64 & 6.81 & 11.22 & 3.16 & 6.08 & 10.97 & 2.43 & 5.62 & 10.38 & 1.02 & 5.29 & 10.06 \\
\hline$S_{2.5}$ & & 3.07 & 8.49 & 12.71 & 2.76 & 6.78 & 11.18 & 2.18 & 6.45 & 10.87 & 1.75 & 5.41 & 10.61 \\
\hline FH-Stereo ${ }_{10}$ & & 2.96 & 7.61 & 11.72 & 3.18 & 6.53 & 10.86 & 2.88 & 5.82 & 10.37 & 1.63 & 5.11 & 10.27 \\
\hline$S_{2,3}$ & & 5. & 9.96 & 80 & 2.89 & 8.55 & 66 & 48 & 6.63 & 37 & 1.06 & 5.87 & 10.78 \\
\hline FH-Stereo 6 & & 4.38 & 9.74 & 15.77 & 2.58 & 8.74 & 3 & 96 & 5.88 & 6 & 72 & 5.45 & 10.71 \\
\hline$S_{2,4}$ & & 5.36 & 9.42 & 15.01 & 2.57 & 8.11 & 12.74 & 2.05 & 6.28 & 12.28 & 1.55 & 5.59 & 10.05 \\
\hline FH-Stereo 8 & & 4.22 & 9.85 & 15.88 & 2.97 & 8.57 & 14.65 & 1.94 & 5.48 & 13.26 & 1.29 & 5.15 & 10.18 \\
\hline$S_{2,5}$ & & 5.8 & 9.47 & 14.92 & 2.71 & 8.44 & & 41 & 6.85 & 4 & 17 & 2 & 10.06 \\
\hline FH-Stereo ${ }_{10}$ & & 4.26 & 9.69 & 15.60 & 2.43 & 8.48 & 14.31 & 1.42 & 5.45 & 13.61 & 1.39 & 5.25 & 10.97 \\
\hline$S_{2,3}$ & & 224 & 9.61 & 11.69 & 1.99 & 7.02 & 11.68 & 1.96 & 6.01 & 1.38 & 1.24 & 5.58 & 9.94 \\
\hline FH-Stereo 6 & & 6.1 & 9. & 81 & 5.48 & 6.51 & 13.46 & 2.22 & 7.71 & 12.16 & 1.26 & 5.12 & 10.29 \\
\hline$S_{2,4}$ & & 3.36 & 10.01 & 14.31 & 3.19 & 8.03 & 12.52 & 2.56 & 6.70 & 12.25 & 1.31 & 5.18 & 10.52 \\
\hline FH-Stereo 8 & $2 \sim \operatorname{Bern}(0.5)$ & 6.76 & 12.46 & 15.51 & 3.46 & 9.37 & 13.15 & 2.17 & 6.17 & 12.06 & 1.48 & 5.16 & 10.23 \\
\hline$S_{2,5}$ & & 4.33 & 9.11 & 12.13 & 3.36 & 6.65 & 11.08 & 2.58 & 6.11 & 10.24 & 1.36 & 5.09 & 10.23 \\
\hline FH-S & & 5.07 & 8.24 & 14.95 & 4.88 & 7.21 & 12.96 & 2.67 & 5.53 & 11.67 & 1.45 & 5.12 & 10.31 \\
\hline$S_{2,3}$ & & 2. & 8.93 & 12 & 2.43 & 6.00 & 1 & 2.5 & 93 & 9. & 1.36 & 5.43 & 10 \\
\hline FH-Stereo $_{6}$ & & 4.8 & 7.62 & 12.64 & 3.86 & 7.12 & 12.87 & 2.97 & 5.78 & 13.97 & 1.92 & 5.91 & 10.59 \\
\hline & & 3.41 & 8.91 & 12.02 & 2.88 & 6.51 & 10.87 & 2.64 & 6.42 & 10.09 & 1.69 & 5.49 & 10.61 \\
\hline FH-Ste & $x_{2} \sim \operatorname{Bern}(0.5)$ & 4.73 & 8.28 & 14.12 & 3.69 & 7.39 & 13.00 & 3.36 & 5.39 & 13.88 & 1.66 & 5.88 & 10.67 \\
\hline & & & 9. & & 3.36 & 6.65 & & 2.58 & 6.11 & & 1.86 & 4.89 & 10.03 \\
\hline FH-Stereo ${ }_{10}$ & & 5.07 & 8.24 & 14.95 & 4.88 & 7.21 & 13.96 & 3.67 & 5.53 & 13.66 & 1.95 & 5.12 & 10.31 \\
\hline
\end{tabular}

Note: $S_{g_{1}, g_{2}}$ is the proposed test with the first partition (deviance) into $g_{1}$ and the second partition into $g_{2}$ groups; FH-Stere ${ }_{G}$ is the Fagerland-Hosmer test with the fitted probabilities from the ordered stereotype model using $G$ groups; and $G=g_{1} \times g_{2}$. 


\section{Statistics}

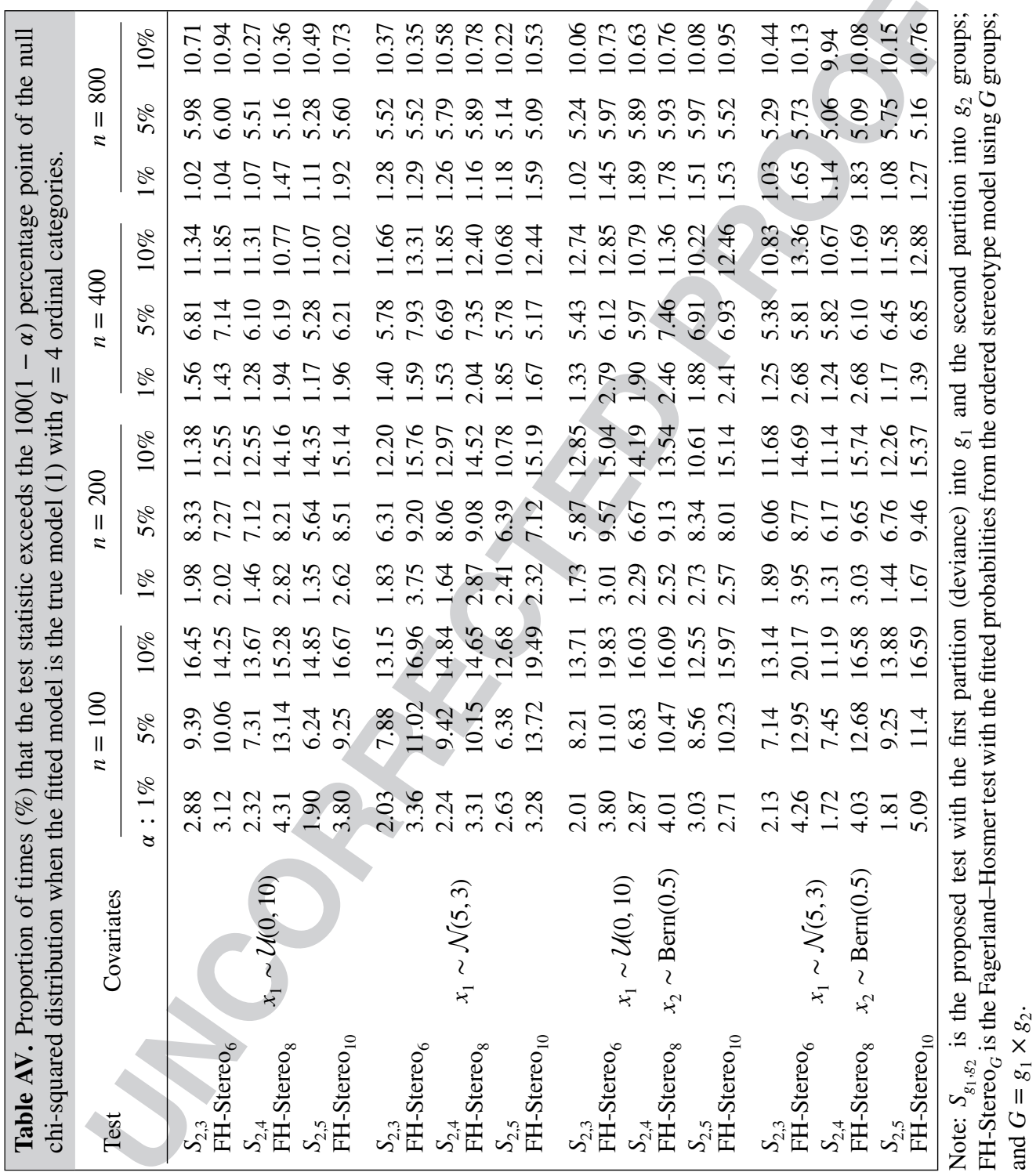




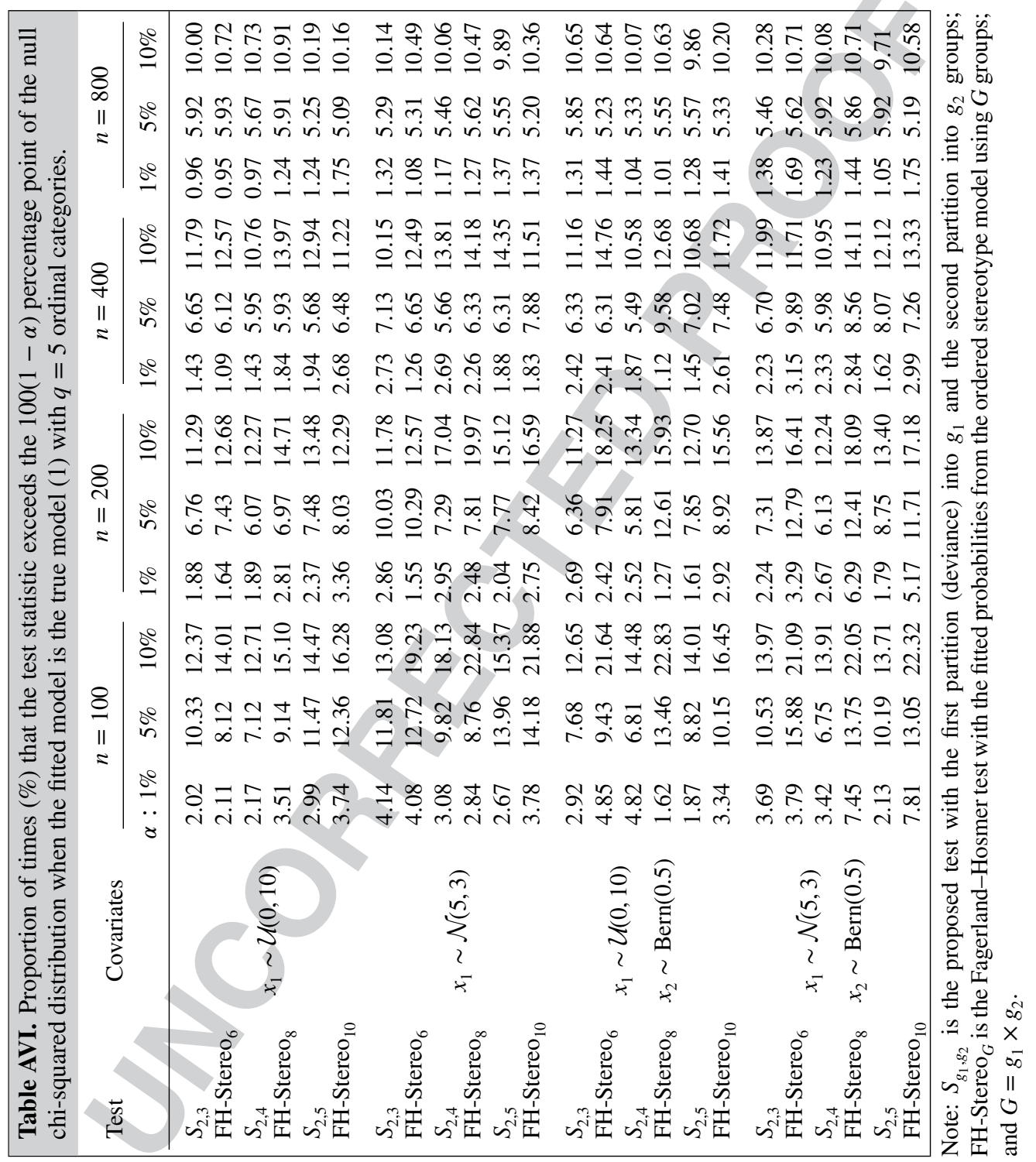




\section{Statistics}

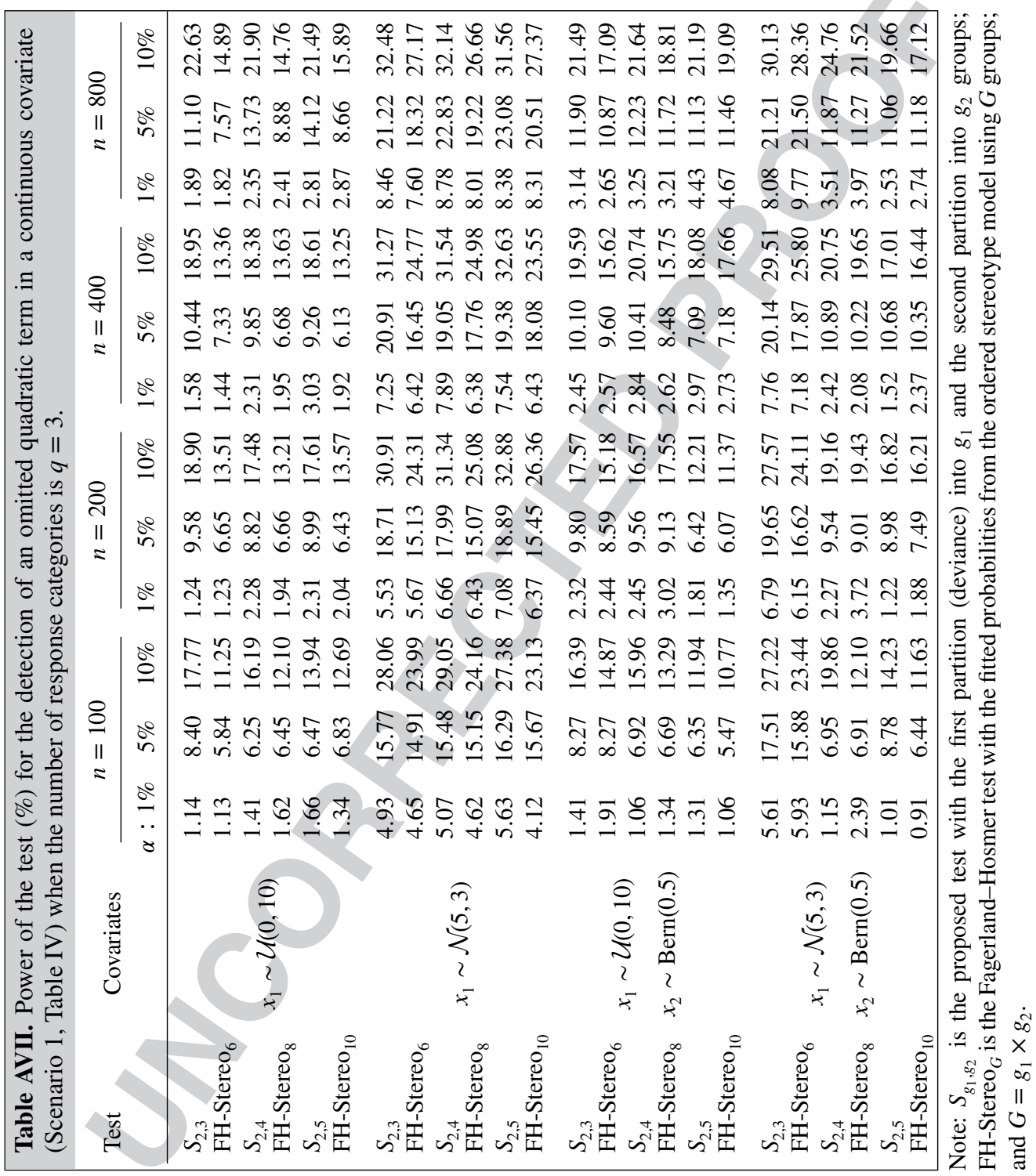




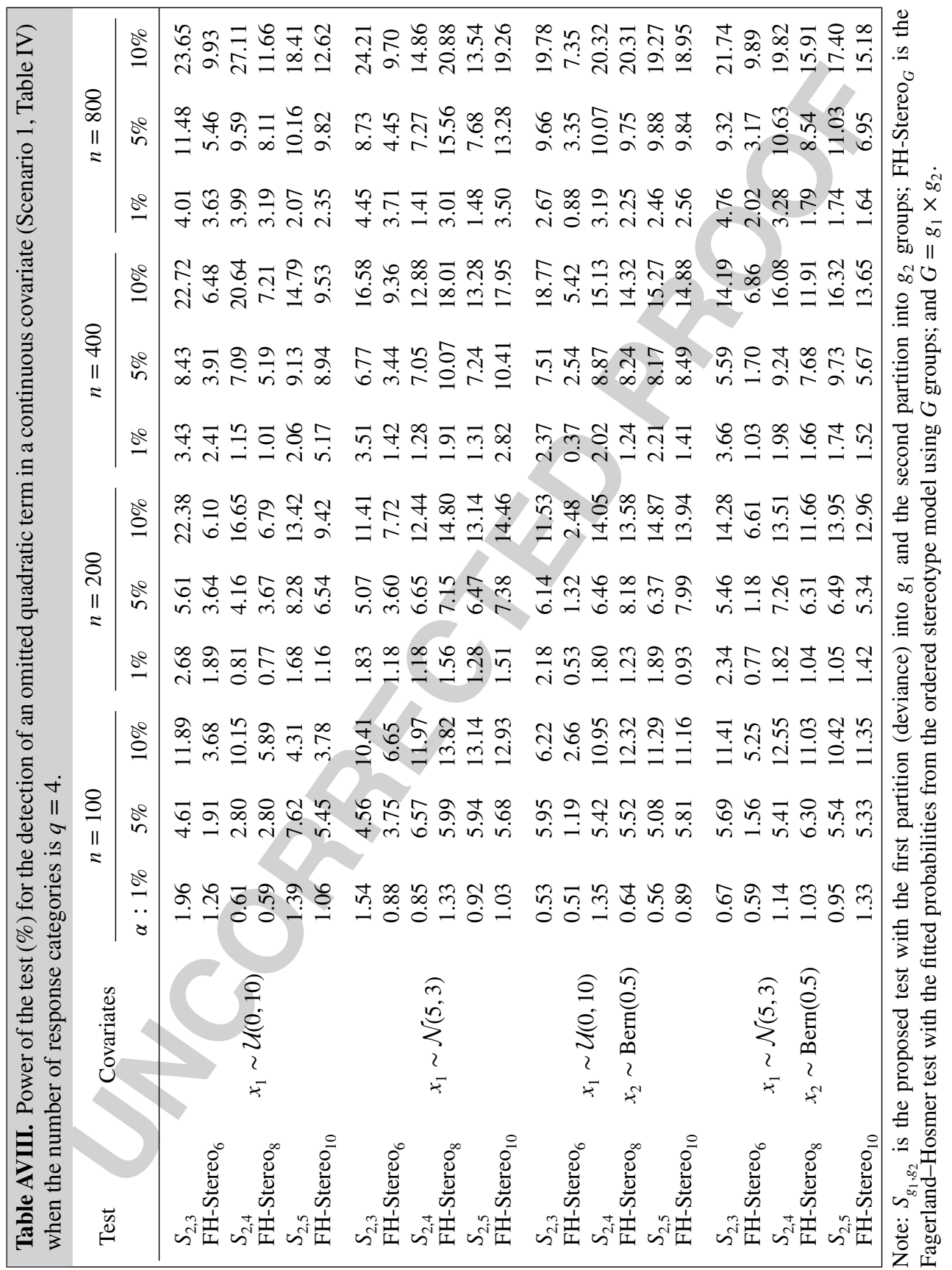




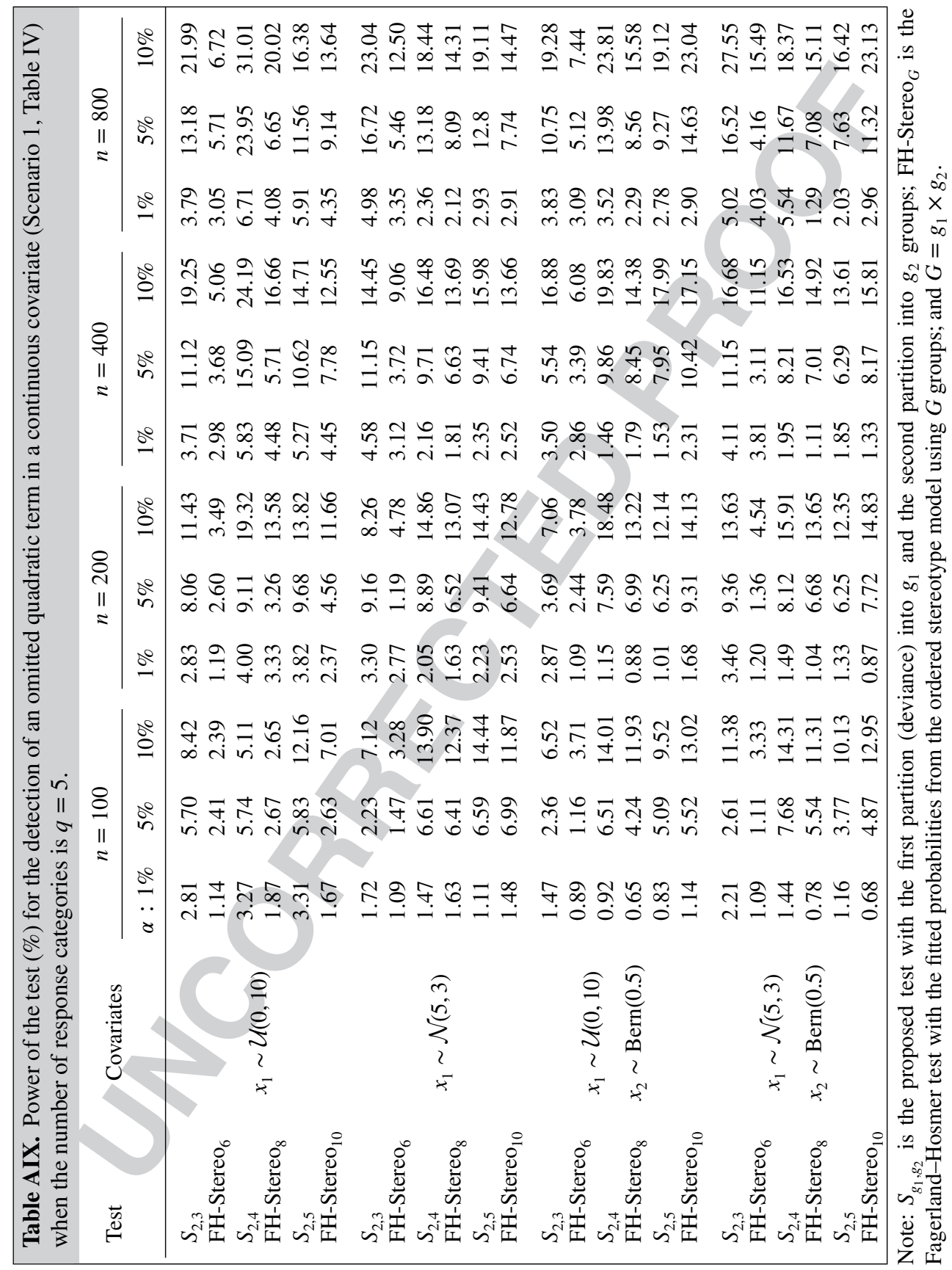




\begin{tabular}{|c|c|c|c|c|c|c|c|c|c|c|c|c|c|}
\hline \multirow{2}{*}{ Test } & \multirow{2}{*}{ Covariates } & \multicolumn{3}{|c|}{$n=100$} & \multicolumn{3}{|c|}{$n=200$} & \multicolumn{3}{|c|}{$n=400$} & \multicolumn{3}{|c|}{$n=800$} \\
\hline & & $\alpha: 1 \%$ & $5 \%$ & $10 \%$ & $1 \%$ & $5 \%$ & $10 \%$ & $1 \%$ & $5 \%$ & $10 \%$ & $1 \%$ & $5 \%$ & $10 \%$ \\
\hline$S_{2,3}$ & & 0.56 & 8.01 & 14.83 & 0.89 & 8.33 & 15.06 & 1.85 & 8.67 & 15.34 & 2.73 & 10.32 & 17.42 \\
\hline FH-Stereo 6 & & 1.23 & 7.37 & 11.75 & 1.81 & 7.99 & 13.56 & 1.95 & 9.55 & 14.64 & 2.46 & 9.82 & 15.44 \\
\hline$S_{2,4}$ & (0) & 0.62 & 8.36 & 13.55 & 0.98 & 8.68 & 13.91 & 1.16 & 9.21 & 14.04 & 1.62 & 9.22 & 14.89 \\
\hline $\mathrm{FH}-\mathrm{Stereo}_{8}$ & $x_{2} \sim \operatorname{Bern}(0.5)$ & 0.87 & 6.27 & 10.94 & 2.02 & 6.73 & 14.18 & 2.11 & 8.39 & 14.19 & 2.16 & 8.65 & 15.66 \\
\hline$S_{2,5}$ & & 1.52 & 6.63 & 10.78 & 1.64 & 7.08 & 13.22 & 2.29 & 9.01 & 15.56 & 2.98 & 9.04 & 15.66 \\
\hline FH-Stereo $_{10}$ & & 1.25 & 6.77 & 12.65 & 1.71 & 7.53 & 14.89 & 1.92 & 8.17 & 16.11 & 1.99 & 8.71 & 16.81 \\
\hline$S_{2,3}$ & & 1.18 & 7.17 & 15.76 & 1.62 & 7.54 & 15.93 & 1.77 & 8.33 & 17.09 & 2.99 & 9.81 & 18.96 \\
\hline $\mathrm{FH}_{-}$Stereo $_{6}$ & 1) & 1.43 & 7.48 & 15.92 & 1.84 & 8.33 & 16.61 & 2.01 & 8.84 & 16.67 & 2.42 & 8.87 & 17.99 \\
\hline$S_{2,4}$ & $x_{1} \sim$ & 1.66 & 8.45 & 13.96 & 1.85 & 8.62 & 14.64 & 2.18 & 8.86 & 16.26 & 3.29 & 8.97 & 16.83 \\
\hline FH-Stereo 8 & $x_{2} \sim \operatorname{Bern}(0.5)$ & 1.46 & 7.48 & 12.80 & 1.66 & 8.01 & 14.43 & 1.73 & 8.62 & 14.77 & 1.85 & 8.86 & 15.47 \\
\hline$S_{2,5}$ & & 1.37 & 7.24 & 14.43 & 1.43 & 7.51 & 15.28 & 1.98 & 8.11 & 15.46 & 2.33 & 8.53 & 16.08 \\
\hline FH-Stereo $_{10}$ & & 1.19 & 4.72 & 12.33 & 1.33 & 7.26 & 13.36 & 1.56 & 7.36 & 15.04 & 1.96 & 8.54 & 17.53 \\
\hline
\end{tabular}

Note: $S_{g_{1}, g_{2}}$ is the proposed test with the first partition (deviance) into $g_{1}$ and the second partition into $g_{2}$ groups; FH-Stereo ${ }_{G}$ is the Fagerland-Hosmer test with the fitted probabilities from the ordered stereotype model using $G$ groups; and $G=g_{1} \times g_{2}$.

\begin{tabular}{|c|c|c|c|c|c|c|c|c|c|c|c|c|c|}
\hline \multirow{2}{*}{ Test } & \multirow{2}{*}{ Covariates } & \multicolumn{3}{|c|}{$n=100$} & \multicolumn{3}{|c|}{$n=200$} & \multicolumn{3}{|c|}{$n=400$} & \multicolumn{3}{|c|}{$n=800$} \\
\hline & & $\alpha: 1 \%$ & $5 \%$ & $10 \%$ & $1 \%$ & $5 \%$ & $10 \%$ & $1 \%$ & $5 \%$ & $10 \%$ & $1 \%$ & $5 \%$ & $10 \%$ \\
\hline$S_{2,3}$ & & & 6.61 & 12.81 & 1.53 & 7.72 & 13.01 & 1.66 & 7.83 & 14.14 & 1.81 & 7.94 & 15.58 \\
\hline FH-Stereo $_{6}$ & & 1.05 & 4.35 & 10.37 & 1.07 & 4.51 & 11.81 & 1.41 & 5.18 & 13.31 & 2.25 & 7.31 & 14.24 \\
\hline$S_{2,4}$ & & 1.36 & 6.39 & 11.85 & 1.59 & 6.52 & 12.34 & 1.62 & 6.78 & 14.36 & 1.71 & 8.22 & 14.29 \\
\hline $\mathrm{FH} \mathrm{StereO}_{8}$ & $x_{2} \sim \operatorname{Bern}(0.5)$ & 0.97 & 5.44 & 10.63 & 1.01 & 5.58 & 11.43 & 1.12 & 5.65 & 11.59 & 1.14 & 5.72 & 12.28 \\
\hline$S_{2,5}$ & & 1.32 & 5.05 & 11.82 & 1.31 & 5.89 & 12.52 & 1.35 & 7.21 & 12.83 & 1.54 & 7.91 & 14.16 \\
\hline FH-Stereo $_{10}$ & & 0.47 & 4.68 & 9.72 & 0.67 & 4.81 & 10.27 & 0.93 & 5.16 & 10.81 & 1.36 & 6.41 & 12.50 \\
\hline$S_{2,3}$ & & 1.06 & 5.77 & 11.71 & 1.18 & 6.61 & 12.09 & 1.33 & 7.17 & 12.78 & 1.50 & 7.76 & 12.93 \\
\hline FH-Stereo $_{6}$ & & 0.45 & 4.07 & 8.35 & 0.91 & 4.06 & 9.82 & 1.17 & 4.86 & 10.21 & 1.42 & 6.51 & 11.86 \\
\hline$S_{2,4}$ & $\mathcal{N}(5,3)$ & 0.86 & 4.57 & 6.71 & 1.25 & 5.81 & 10.53 & 1.41 & 7.48 & 13.16 & 1.63 & 8.64 & 15.62 \\
\hline $\mathrm{FH}-$ Stereo $_{8}$ & $x_{2} \sim \operatorname{Bern}(0.5)$ & 0.65 & 4.06 & 5.31 & 0.91 & 4.34 & 8.62 & 1.23 & 4.96 & 9.88 & 1.35 & 6.17 & 10.96 \\
\hline$S_{2,5}$ & & 0.95 & 4.54 & 10.64 & 1.07 & 4.93 & 10.71 & 1.08 & 5.01 & 11.91 & 1.16 & 5.93 & 12.80 \\
\hline FH-Stereo $_{10}$ & & 0.36 & 3.96 & 9.63 & 0.64 & 4.15 & 9.67 & 0.66 & 4.36 & 9.90 & 0.84 & 4.88 & 10.31 \\
\hline
\end{tabular}

Note: $S_{g_{1}, g_{2}}$ is the proposed test with the first partition (deviance) into $g_{1}$ and the second partition into $g_{2}$ groups; FH-Stereo ${ }_{G}$ is the Fagerland-Hosmer test with the fitted probabilities from the ordered stereotype model using $G$ groups; and $G=g_{1} \times g_{2}$. 


\begin{tabular}{|c|c|c|c|c|c|c|c|c|c|c|c|c|c|}
\hline \multirow{2}{*}{ Test } & \multirow{2}{*}{ Covariates } & \multicolumn{3}{|c|}{$n=100$} & \multicolumn{3}{|c|}{$n=200$} & \multicolumn{3}{|c|}{$n=400$} & \multicolumn{3}{|c|}{$n=800$} \\
\hline & & $\alpha: 1 \%$ & $5 \%$ & $10 \%$ & $1 \%$ & $5 \%$ & $10 \%$ & $1 \%$ & $5 \%$ & $10 \%$ & $1 \%$ & $5 \%$ & $10 \%$ \\
\hline$S_{2,3}$ & & 0.47 & 6.64 & 8.42 & 1.55 & 7.05 & 13.04 & 1.89 & 8.38 & 16.81 & 3.08 & 14.19 & 21.48 \\
\hline $\mathrm{FH}_{-S^{-}}$tereo $_{6}$ & $\sim \mathcal{U}(0,10)$ & 0.45 & 6.51 & 7.38 & 0.75 & 7.19 & 12.96 & 1.35 & 8.77 & 14.09 & 2.15 & 14.89 & 15.22 \\
\hline & & 1.63 & 6.54 & 12.88 & 2.17 & 6.57 & 12.98 & 2.36 & 7.46 & 13.33 & 3.97 & 8.62 & 26.67 \\
\hline $\mathrm{FH}_{-S^{-}}$tereo $_{8}$ & $x_{2} \sim \operatorname{Bern}(0.5)$ & 0.94 & 5.49 & 13.33 & 1.63 & 7.07 & 14.11 & 1.88 & 7.32 & 15.56 & 3.00 & 8.12 & 16.03 \\
\hline & & 0.74 & 4.23 & 8.33 & 1.12 & 4.76 & 9.02 & 3.70 & 4.83 & 13.04 & 4.08 & 14.81 & 24.43 \\
\hline FH-Stereo $_{10}$ & & 0.56 & 4.35 & 8.27 & 1.48 & 5.56 & 15.22 & 1.85 & 9.15 & 16.61 & 2.33 & 14.29 & 21.33 \\
\hline$S_{2,3}$ & & 1.61 & 2.94 & 6.08 & 2.27 & 4.42 & 6.49 & 6.09 & 5.56 & 9.49 & 9.19 & 15.65 & 22.55 \\
\hline FH-Stereo $_{6}$ & & 1.52 & 2.78 & 5.88 & 2.08 & 4.12 & 10.22 & 5.45 & 6.19 & 10.39 & 7.07 & 8.33 & 15.49 \\
\hline$S_{2,4}$ & & 1.12 & 3.29 & 4.55 & 1.52 & 4.55 & 10.00 & 1.94 & 7.77 & 12.51 & 2.99 & 14.17 & 20.83 \\
\hline $\mathrm{FH}^{-S_{t e r e o}} 8$ & $x_{2} \sim \operatorname{Bern}(0.5)$ & 0.68 & 1.97 & 1.79 & 1.21 & 5.56 & 8.33 & 1.63 & 5.56 & 12.53 & 2.19 & 6.06 & 18.18 \\
\hline & & 1.79 & 2.27 & 2.22 & 2.41 & 4.29 & 3.21 & 6.46 & 10.53 & 11.76 & 8.19 & 12.78 & 21.11 \\
\hline $\mathrm{FH}^{-S_{\text {Stereo }}}{ }_{10}$ & & 1.85 & 3.79 & 3.75 & 1.33 & 5.26 & 7.05 & 4.19 & 7.14 & 7.78 & 6.96 & 8.41 & 19.52 \\
\hline
\end{tabular}

Note: $S_{g_{1}, g_{2}}$ is the proposed test with the first partition (deviance) into $g_{1}$ and the second partition into $g_{2}$ groups; FH-Stereo ${ }_{G}$ is the Fagerland-Hosmer test with the fitted probabilities from the ordered stereotype model using $G$ groups; and $G=g_{1} \times g_{2}$.

Table AXIII. Power of the test (\%) to detect the wrong functional form of a continuous covariate (Scenario 3 , Table IV), when the number of response categories is $q=3$.

\begin{tabular}{|c|c|c|c|c|c|c|c|c|c|c|c|c|c|}
\hline \multirow{2}{*}{ Test } & \multirow{2}{*}{ Covariates } & \multicolumn{3}{|c|}{$n=100$} & \multicolumn{3}{|c|}{$n=200$} & \multicolumn{3}{|c|}{$n=400$} & \multicolumn{3}{|c|}{$n=800$} \\
\hline & & $\alpha: 1 \%$ & $5 \%$ & $10 \%$ & $1 \%$ & $5 \%$ & $10 \%$ & $1 \%$ & $5 \%$ & $10 \%$ & $1 \%$ & $5 \%$ & $10 \%$ \\
\hline$S_{2,3}$ & & 4.35 & 13.95 & 23.67 & 4.91 & 14.75 & 24.02 & 5.73 & 16.95 & 24.62 & 5.89 & 22.22 & 25.25 \\
\hline $\mathrm{FH}^{-S_{\text {Stereo }}} 6$ & $x_{1}$ & 3.94 & 15.76 & 20.11 & 4.35 & 16.67 & 22.26 & 4.38 & 18.21 & 26.03 & 5.72 & 20.95 & 27.65 \\
\hline$S_{2,4}$ & 1 & 3.51 & 11.68 & 20.75 & 3.64 & 12.82 & 21.84 & 4.25 & 13.48 & 23.31 & 4.57 & 15.09 & 25.12 \\
\hline $\mathrm{FH}_{-S t e r e o}$ & $x_{2} \sim \operatorname{Bern}(0.5)$ & 3.51 & 12.23 & 16.60 & 3.98 & 12.82 & 21.65 & 4.25 & 14.21 & 21.96 & 4.43 & 14.74 & 23.55 \\
\hline & & 3.53 & 12.56 & 20.14 & 4.15 & 12.87 & 20.93 & 5.29 & 15.35 & 21.82 & 6.01 & 15.61 & 27.24 \\
\hline FH-Stereo $_{10}$ & & 3.03 & 11.03 & 19.79 & 3.53 & 12.11 & 20.59 & 4.78 & 12.38 & 21.03 & 5.54 & 13.77 & 22.54 \\
\hline
\end{tabular}

Note: $S_{g_{1}, g_{2}}$ is the proposed test with the first partition (deviance) into $g_{1}$ and the second partition into $g_{2}$ groups; FH-Stereo ${ }_{G}$ is the Fagerland-Hosmer test with the fitted probabilities from the ordered stereotype model using $G$ groups; and $G=g_{1} \times g_{2}$.

Table AXIV. Power of the test (\%) to detect the wrong functional form of a continuous covariate (Scenario 3, Table IV), when the number of response categories is $q=4$.

\begin{tabular}{|c|c|c|c|c|c|c|c|c|c|c|c|c|c|}
\hline \multirow{2}{*}{ Test } & \multirow{2}{*}{ Covariates } & \multicolumn{3}{|c|}{$n=100$} & \multicolumn{3}{|c|}{$n=200$} & \multicolumn{3}{|c|}{$n=400$} & \multicolumn{3}{|c|}{$n=800$} \\
\hline & & $\alpha: 1 \%$ & $5 \%$ & $10 \%$ & $1 \%$ & $5 \%$ & $10 \%$ & $1 \%$ & $5 \%$ & $10 \%$ & $1 \%$ & $5 \%$ & $10 \%$ \\
\hline$S_{2,3}$ & & 2.48 & 10.34 & 20.41 & 2.66 & 10.97 & 20.56 & 4.12 & 11.51 & 22.71 & 4.25 & 14.99 & 24.41 \\
\hline $\mathrm{FH}_{- \text {Stereo }_{6}}$ & $x_{1} \sim \mathcal{U}(0,10)$ & 2.31 & 8.13 & 16.47 & 2.58 & 9.06 & 17.48 & 2.80 & 9.61 & 18.12 & 2.88 & 10.44 & 18.21 \\
\hline$S_{2,4}$ & & 2.02 & 10.27 & 17.91 & 2.31 & 10.31 & 19.79 & 2.71 & 11.32 & 21.51 & 3.44 & 12.82 & 21.82 \\
\hline $\mathrm{FH}_{-S t e r e o}$ & $x_{2} \sim \operatorname{Bern}(0.5)$ & 2.12 & 7.96 & 14.18 & 2.15 & 8.45 & 14.76 & 2.30 & 9.68 & 16.67 & 2.67 & 9.99 & 17.42 \\
\hline$S_{2,5}$ & & 2.07 & 8.97 & 17.12 & 2.35 & 10.31 & 17.12 & 2.91 & 10.91 & 17.50 & 2.89 & 11.00 & 20.12 \\
\hline $\mathrm{FH}$ Stereo $_{10}$ & & 1.62 & 7.90 & 13.72 & 2.07 & 8.06 & 13.86 & 2.36 & 8.99 & 14.66 & 3.34 & 9.32 & 16.12 \\
\hline
\end{tabular}

Note: $S_{g_{1}, g_{2}}$ is the proposed test with the first partition (deviance) into $g_{1}$ and the second partition into $g_{2}$ groups; FH-Stereo ${ }_{G}$ is the Fagerland-Hosmer test with the fitted probabilities from the ordered stereotype model using $G$ groups; and $G=g_{1} \times g_{2}$. 
Table AXV. Power of the test (\%) to detect the wrong functional form of a continuous covariate (Scenario 3, Table IV), when the number of response categories is $q=5$.

\begin{tabular}{|c|c|c|c|c|c|c|c|c|c|c|c|c|c|}
\hline \multirow{2}{*}{ Test } & \multirow{2}{*}{ Covariates } & \multicolumn{3}{|c|}{$n=100$} & \multicolumn{3}{|c|}{$n=200$} & \multicolumn{3}{|c|}{$n=400$} & \multicolumn{3}{|c|}{$n=800$} \\
\hline & & $\alpha: 1 \%$ & $5 \%$ & $10 \%$ & $1 \%$ & $5 \%$ & $10 \%$ & $1 \%$ & $5 \%$ & $10 \%$ & $1 \%$ & $5 \%$ & $10 \%$ \\
\hline$S_{2,3}$ & & 2.35 & 11.43 & 18.63 & 2.44 & 12.63 & 18.96 & 5.15 & 15.16 & 27.42 & 7.07 & 21.42 & 31.58 \\
\hline FH-Stereo $_{6}$ & $x_{1} \sim \mathcal{U}(0,10)$ & 2.02 & 10.11 & 16.48 & 2.28 & 11.14 & 16.84 & 3.87 & 11.97 & 22.11 & 5.18 & 14.90 & 26.13 \\
\hline$S_{2,4}$ & & 2.14 & 10.16 & 16.67 & 3.36 & 11.29 & 19.13 & 5.25 & 17.24 & 26.53 & 7.23 & 21.43 & 30.43 \\
\hline FH-Stereo $_{8}$ & $x_{2} \sim \operatorname{Bern}(0.5)$ & 1.95 & 8.29 & 13.67 & 2.14 & 9.67 & 14.57 & 4.50 & 12.69 & 20.13 & 5.09 & 13.67 & 24.33 \\
\hline$S_{2,5}$ & & 2.35 & 11.11 & 15.79 & 3.53 & 12.45 & 19.88 & 5.75 & 16.64 & 28.11 & 7.97 & 22.00 & 31.23 \\
\hline FH-Stereo $_{10}$ & & 1.51 & 9.46 & 13.47 & 2.18 & 8.19 & 13.39 & 4.24 & 11.54 & 21.12 & 5.17 & 13.35 & 25.08 \\
\hline
\end{tabular}

Note: $S_{g_{1}, g_{2}}$ is the proposed test with the first partition (deviance) into $g_{1}$ and the second partition into $g_{2}$ groups; FH-Stereo ${ }_{G}$ is the Fagerland-Hosmer test with the fitted probabilities from the ordered stereotype model using $G$ groups; and $G=g_{1} \times g_{2}$.

Table AXVI. Power of the test (\%) to detect the wrong ordinal response assumption (nominal response variable, Scenario 4, Table IV), when the number of response categories is $q=3$.

\begin{tabular}{|c|c|c|c|c|c|c|c|c|c|c|c|c|c|}
\hline \multirow{2}{*}{ Test } & \multirow{2}{*}{ Covariates } & \multicolumn{3}{|c|}{$n=100$} & \multicolumn{3}{|c|}{$n=200$} & \multicolumn{3}{|c|}{$n=400$} & \multicolumn{3}{|c|}{$n=800$} \\
\hline & & $\alpha: 1 \%$ & $5 \%$ & $10 \%$ & $1 \%$ & $5 \%$ & $10 \%$ & $1 \%$ & $5 \%$ & $10 \%$ & $1 \%$ & $5 \%$ & $10 \%$ \\
\hline$S_{2,3}$ & & 1.18 & 5.52 & 13.31 & 1.55 & 9.06 & 13.64 & 2.17 & 10.07 & 15.65 & 2.82 & 10.69 & 17.28 \\
\hline FH-Stereo 6 & & 1.95 & 6.11 & 21.28 & 4.79 & 10.53 & 23.73 & 5.33 & 13.79 & 25.21 & 5.43 & 23.39 & 25.45 \\
\hline$S_{2,4}$ & & 0.79 & 6.57 & 12.69 & 0.86 & 6.69 & 15.95 & 1.44 & 8.28 & 17.17 & 2.03 & 11.18 & 18.49 \\
\hline $\mathrm{FH}_{-S t e r e O}$ & & 0.86 & 11.86 & 15.67 & 1.58 & 14.64 & 16.44 & 4.72 & 14.83 & 19.95 & 4.94 & 16.45 & 25.93 \\
\hline$S_{2,5}$ & & 1.06 & 5.31 & 9.49 & 1.39 & 6.11 & 13.33 & 2.58 & 6.52 & 16.34 & 3.71 & 8.16 & 17.42 \\
\hline FH-Stereo ${ }_{10}$ & & 1.48 & 10.63 & 13.14 & 2.54 & 10.69 & 17.78 & 3.25 & 13.04 & 21.57 & 4.67 & 16.62 & 24.62 \\
\hline$S_{2,3}$ & & 1.44 & 7.23 & 91 & 2.23 & 8. & 18.38 & 2.54 & 9. & 1 & 9 & & 22.13 \\
\hline FH-Stereo $_{6}$ & & 3.31 & 10.06 & 21.62 & 3.43 & 13.15 & 25.62 & 3.75 & 14.68 & 26.35 & 4.11 & 18.52 & 27.32 \\
\hline$S_{2,4}$ & & 1.34 & 4.02 & 14.08 & 2.27 & 6.92 & 14.74 & 4.38 & 7.46 & 15.98 & 6.33 & 9.72 & 17.66 \\
\hline $\mathrm{FH}-\mathrm{Stereo}_{8}$ & & 2.27 & 10.56 & 17.23 & 2.50 & 11.83 & 23.08 & 2.74 & 13.79 & 25.44 & 3.76 & 14.37 & 25.75 \\
\hline$S_{2,5}$ & & 1.17 & 6.71 & 13.12 & 1.91 & 8.42 & 16.67 & 2.03 & 8.45 & 16.87 & 2.31 & 11.18 & 18.99 \\
\hline FH-Stereo ${ }_{10}$ & & 2.03 & 10.44 & 18.87 & 2.34 & 11.18 & 21.69 & 2.73 & 11.95 & 23.64 & 2.89 & 12.47 & 26.82 \\
\hline$S_{2,3}$ & & 1.45 & 7.58 & 12.37 & 3.03 & 8.97 & 13.50 & 3.47 & 9.09 & 13.69 & 3.83 & 9.68 & 18.56 \\
\hline $\mathrm{FH}-$ Stereo $_{6}$ & & 2.98 & 12.16 & 22.89 & 3.28 & 12.33 & 23.47 & 3.51 & 13.44 & 23.66 & 7.07 & 18.18 & 25.48 \\
\hline$S_{2,4}$ & & 0.67 & 2.81 & 14.84 & 1.06 & 6.84 & 15.78 & 1.99 & 8.79 & 17.54 & 2.56 & 11.82 & 18.13 \\
\hline $\mathrm{FH}-$ Stereo $_{8}$ & $x_{2} \sim \operatorname{Bern}(0.5)$ & 1.99 & 9.87 & 17.09 & 2.12 & 11.33 & 19.29 & 2.23 & 15.73 & 25.45 & 5.77 & 15.77 & 25.82 \\
\hline$S_{2,5}$ & & 0.51 & 5.26 & 10.61 & 0.65 & 6.62 & 13.28 & 0.92 & 7.36 & 14.06 & 1.16 & 7.92 & 15.11 \\
\hline FH-Stereo ${ }_{10}$ & & 2.39 & 10.78 & 18.31 & 3.44 & 12.44 & 18.54 & 3.47 & 15.23 & 19.59 & 6.06 & 20.25 & 24.02 \\
\hline$S_{2,3}$ & & & & & 2.11 & & 18.21 & 2.66 & 6 & 6 & 8 & 12.21 & 27.18 \\
\hline FH-Stereo $_{6}$ & & 2.81 & 13.12 & 21.30 & 3.17 & 15.71 & 25.38 & 6.47 & 16.41 & 35.55 & 7.45 & 19.19 & 33.75 \\
\hline$S_{2,4}$ & & 1.11 & 7.52 & 16.60 & 1.75 & 9.04 & 18.91 & 2.76 & 9.67 & 24.07 & 3.02 & 11.74 & 24.24 \\
\hline $\mathrm{FH}_{-S t e r e o}$ & $x_{2} \sim \operatorname{Bern}(0.5)$ & 4.14 & 10.18 & 20.87 & 4.27 & 11.79 & 23.30 & 4.95 & 13.62 & 26.77 & 5.26 & 19.77 & 28.24 \\
\hline$S_{2,5}$ & & 0.47 & 9.09 & 14.47 & 0.68 & 9.14 & 15.96 & 1.55 & 9.74 & 19.04 & 2.73 & 13.13 & 22.29 \\
\hline $\mathrm{FH}^{-S_{\text {Stereo }}}{ }_{10}$ & & 3.11 & 12.63 & 19.36 & 3.18 & 13.32 & 20.19 & 3.24 & 13.45 & 25.69 & 7.43 & 17.65 & 25.89 \\
\hline
\end{tabular}

Note: $S_{g_{1}, g_{2}}$ is the proposed test with the first partition (deviance) into $g_{1}$ and the second partition into $g_{2}$ groups; FH-Stereo ${ }_{G}$ is the Fagerland-Hosmer test with the fitted probabilities from the ordered stereotype model using $G$ groups; and $G=g_{1} \times g_{2}$.

A.2.4. Wrong ordinal response assumption (nominal response variable). Tables AXVI-AXVIII present power of the test to detect the wrong ordinal response assumption (nominal response variable, Scenario 4, Table IV), when the data were simulated from a baseline-category logit model with the response category of $q=3,4,5$, respectively. power to detect the missing interaction term between a two dichotomous covariates when the data were simulated from Scenario 5 in Table IV with the response category of $q=3,4,5$, respectively. 


\section{Statistics}

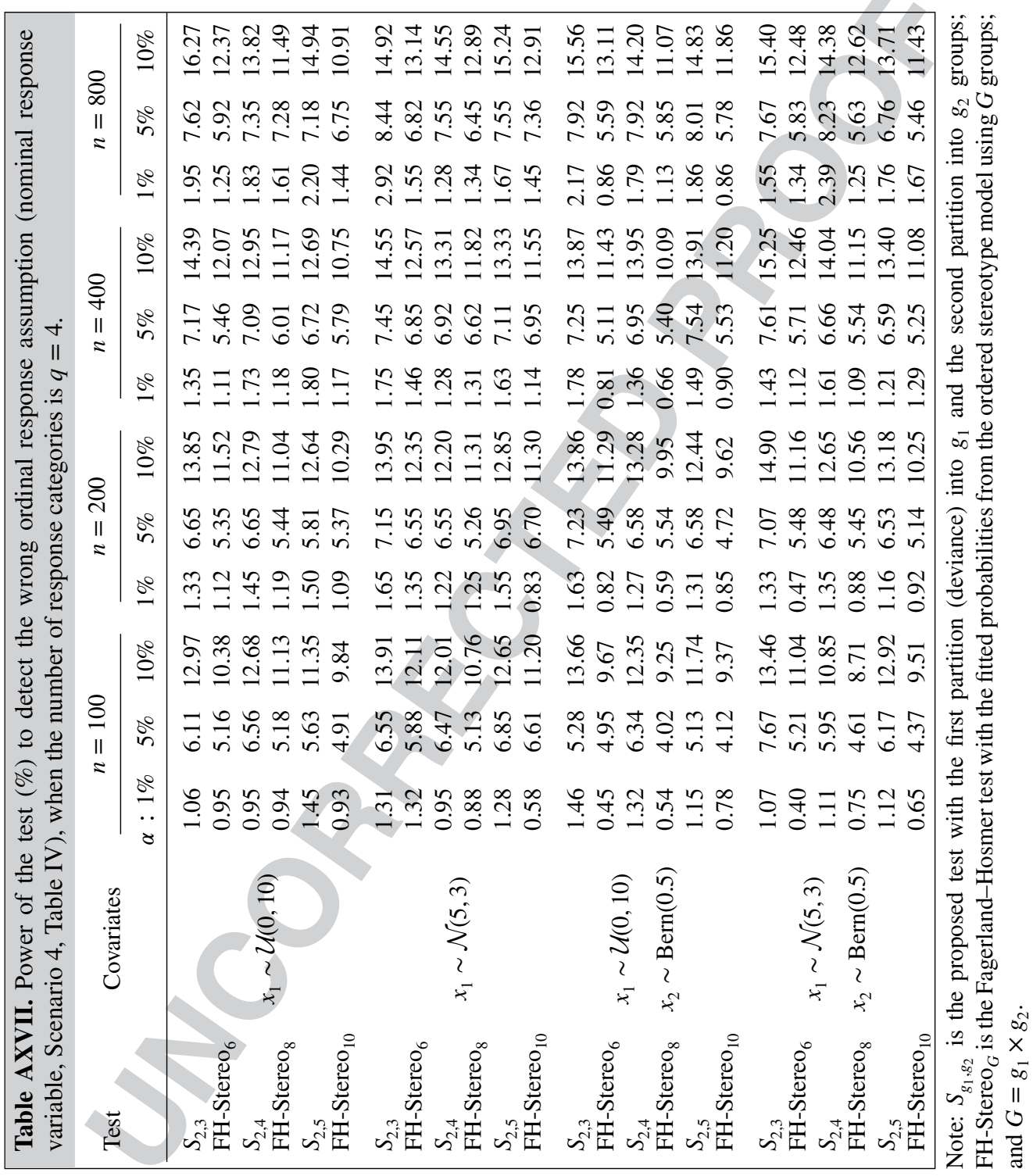


Table AXVIII. Power of the test (\%) to detect the wrong ordinal response assumption (nominal response variable, Scenario 4, Table IV), when the number of response categories is $q=5$.

\begin{tabular}{|c|c|c|c|c|c|c|c|c|c|c|c|c|c|}
\hline \multirow{2}{*}{ Test } & \multirow{2}{*}{ Covariates } & \multicolumn{3}{|c|}{$n=100$} & \multicolumn{3}{|c|}{$n=200$} & \multicolumn{3}{|c|}{$n=400$} & \multicolumn{3}{|c|}{$n=800$} \\
\hline & & $\alpha: 1 \%$ & $5 \%$ & $10 \%$ & $1 \%$ & $5 \%$ & $10 \%$ & $1 \%$ & $5 \%$ & $10 \%$ & $1 \%$ & $5 \%$ & $10 \%$ \\
\hline$S_{2,3}$ & & 1.99 & 6.77 & 14.08 & 2.15 & 7.58 & 14.69 & 2.56 & 8.58 & 15.67 & 3.45 & 9.47 & 16.03 \\
\hline FH-Stereo 6 & & 1.21 & 7.28 & 12.18 & 1.92 & 7.36 & 15.95 & 2.48 & 10.65 & 19.35 & 3.45 & 13.02 & 21.15 \\
\hline & $x=\mathcal{Z}\left(\begin{array}{ll}0 & 10\end{array}\right.$ & 0.93 & 4.02 & 12.18 & 1.53 & 6.07 & 12.46 & 1.72 & 6.63 & 15.28 & 2.06 & 8.96 & 22.46 \\
\hline FH-Stereo ${ }_{8}$ & & 1.48 & 7.42 & 14.34 & 1.53 & 7.47 & 15.97 & 1.55 & 7.71 & 16.59 & 3.45 & 14.92 & 17.65 \\
\hline$S_{2,5}$ & & 1.27 & 4.43 & 10.41 & 1.53 & 5.57 & 10.82 & 1.61 & 5.89 & 12.03 & 1.96 & 6.13 & 16.56 \\
\hline FH-Stereo 10 & & 0.54 & 7.07 & 12.47 & 1.18 & 7.08 & 14.97 & 1.31 & 7.09 & 15.46 & 3.18 & 12.66 & 22.15 \\
\hline$S_{2,3}$ & & 1.28 & 6.06 & 12.35 & 1.45 & 6.28 & 13.09 & 1.56 & 6.78 & 15. & 1.98 & 8.07 & 16.58 \\
\hline FH-Stereo $_{6}$ & & 1.54 & 9.92 & 15.18 & 2.57 & 11.18 & 19.03 & 5.21 & 12.83 & 22.22 & 5.85 & 13.56 & 25.65 \\
\hline$S_{2,4}$ & & 0.49 & 5.45 & 9.34 & 0.74 & 7.32 & 10.63 & 1.12 & 8.27 & 13.74 & 3.65 & 8.61 & 14.31 \\
\hline FH-Stereo ${ }_{8}$ & $x$ & 0.42 & 7.86 & 17.21 & 1.96 & 7.97 & 20.45 & 2.08 & 12.68 & 23.72 & 2.95 & 13.88 & 25.57 \\
\hline$S_{2,5}$ & & 1.05 & 5.38 & 9.89 & 1.47 & 7.25 & 11.63 & 1.57 & 7.56 & 12.53 & 1.99 & 11.66 & 13.27 \\
\hline $\mathrm{FH}$ Stereo $_{10}$ & & 2.11 & 5.83 & 13.27 & 2.31 & 8.51 & 16.48 & 3.98 & 9.85 & 21.43 & 4.71 & 12.79 & 26.16 \\
\hline$S_{2,3}$ & & 1.03 & 4.97 & 14.53 & 1.12 & 7.36 & 14.85 & 1.26 & 7.43 & 16.09 & 2.47 & 9.14 & 16.34 \\
\hline FH-Stereo $_{6}$ & & 1.47 & 7.36 & 10.52 & 1.54 & 7.45 & 15.38 & 1.57 & 8.17 & 19.88 & 5.03 & 9.14 & 21.57 \\
\hline$S_{2,4}$ & & 0.67 & 4.55 & 10.73 & 1.23 & 6.80 & 14.14 & 2.31 & 7.13 & 14.21 & 3.24 & 12.55 & 18.13 \\
\hline FH-Stereo 8 & $x_{2} \sim \operatorname{Bern}(0.5)$ & 0.54 & 4.55 & 10.11 & 0.63 & 6.25 & 15.23 & 1.23 & 6.56 & 18.08 & 4.37 & 7.24 & 20.47 \\
\hline$S_{2,5}$ & & 0.90 & 3.03 & 9.09 & 1.74 & 4.49 & 12.71 & 1.96 & 6.36 & 13.53 & 2.31 & 7.86 & 14.79 \\
\hline FH-Stereo ${ }_{10}$ & & 0.58 & 7.09 & 11.97 & 0.68 & 7.64 & 13.75 & 1.47 & 9.09 & 14.77 & 1.74 & 9.83 & 20.44 \\
\hline$S_{2,3}$ & & 0.51 & 5.52 & 11.94 & 1.44 & 5.05 & 12.24 & 1.47 & 5.37 & 13.69 & 3.01 & 7.88 & 14.65 \\
\hline FH-Stereo $_{6}$ & & 1.47 & 7.16 & 12.44 & 1.96 & 8.05 & 17.35 & 2.34 & 8.33 & 19.05 & 3.08 & 8.89 & 20.38 \\
\hline$S_{2,4}$ & $x_{1} \sim \mathcal{N}(5,3)$ & 1.23 & 4.11 & 12.05 & 1.54 & 7.22 & 12.08 & 2.41 & 8.39 & 13.56 & 3.08 & 9.68 & 15.47 \\
\hline FH-Stereo $_{8}$ & $x_{2} \sim \operatorname{Bern}(0.5)$ & 1.85 & 4.67 & 15.69 & 2.45 & 8.51 & 16.44 & 2.95 & 9.68 & 18.79 & 3.08 & 15.15 & 20.44 \\
\hline$S_{2,5}$ & & 0.58 & 4.19 & 7.59 & 0.64 & 6.43 & 11.39 & 1.21 & 6.76 & 11.41 & 1.61 & 6.86 & 12.29 \\
\hline $\mathrm{FH}_{-S t e r e o}{ }_{10}$ & & 0.64 & 7.02 & 14.54 & 1.07 & 8.53 & 16.39 & 2.18 & 10.99 & 17.09 & 2.33 & 12.25 & 18.44 \\
\hline
\end{tabular}

Note: $S_{g_{1}, g_{2}}$ is the proposed test with the first partition (deviance) into $g_{1}$ and the second partition into $g_{2}$ groups; FH-Stereo ${ }_{G}$ is the Fagerland-Hosmer test with the fitted probabilities from the ordered stereotype model using $G$ groups; and $G=g_{1} \times g_{2}$.

Table AXIX. Power of the test $(\%)$ for the detection of omission of an interaction term between two dichotomous covariates (Scenario 5, Table IV) when the number of response categories is $q=3$.

\begin{tabular}{|c|c|c|c|c|c|c|c|c|c|c|c|c|c|}
\hline \multirow{2}{*}{ Test } & \multirow{2}{*}{ Covariates } & \multicolumn{3}{|c|}{$n=100$} & \multicolumn{3}{|c|}{$n=200$} & \multicolumn{3}{|c|}{$n=400$} & \multicolumn{3}{|c|}{$n=800$} \\
\hline & & $\alpha: 1 \%$ & $5 \%$ & $10 \%$ & $1 \%$ & $5 \%$ & $10 \%$ & $1 \%$ & $5 \%$ & $10 \%$ & $1 \%$ & $5 \%$ & $10 \%$ \\
\hline & & 5.75 & 16.69 & 26.45 & 14.55 & 47.69 & 57.05 & 44.55 & 82.05 & 91.25 & 53.75 & 90.25 & 94.75 \\
\hline FH-Stereo 6 & & 2.55 & 11.44 & 19.82 & 5.35 & 18.24 & 28.75 & 18.25 & 39.91 & 37.35 & 27.17 & 51.95 & 74.56 \\
\hline$S_{2,4}$ & & 3.78 & 15.55 & 27.95 & 55 & 5 & 7 & 5 & 79.55 & 7 & 91 & 82.77 & 1.07 \\
\hline FH-Stereo $_{8}$ & & 2.15 & 10.24 & 18.45 & 4.43 & 15.88 & 23.44 & 15.77 & 37.15 & 30.95 & 26.99 & 48.63 & 70.01 \\
\hline$S_{2,5}$ & & 2.65 & 13.65 & 25.65 & 8.12 & 40.75 & 50.55 & 27.26 & 68.66 & 80.38 & 31.07 & 73.56 & 88.48 \\
\hline $\mathrm{FH}^{-S_{\text {Stereo }}}{ }_{10}$ & & 1.45 & 9.25 & 18.07 & 3.04 & 14.57 & 24.63 & 10.63 & 33.06 & 32.29 & 17.09 & 43.2 & 68.77 \\
\hline & & 0.68 & 20. & 22. & 66 & 25 & & & 37 & & 8 & 78 & 88.6 \\
\hline FH-Stereo $_{6}$ & & 0.74 & 10.37 & 17.34 & 2.52 & 17.78 & 19.56 & 15.52 & 28.61 & 43.18 & 26.45 & 46.35 & 66.35 \\
\hline & & 4.35 & 13.57 & 25.15 & 13.34 & 40.5 & 49.53 & 44.78 & 68.2 & 78.78 & 50.97 & 72.99 & 86.71 \\
\hline FH-Stereo $_{8}$ & $x$ & 1.73 & 8.92 & 17.34 & 3.66 & 14.08 & 23.62 & 12.83 & 31.96 & 31.03 & 20.55 & 41.76 & 66.12 \\
\hline & & 2.85 & 14.35 & 24.75 & 8.78 & 42.85 & 48.71 & 29.52 & 72.19 & 77.45 & 33.63 & 77.27 & 85.22 \\
\hline FH-Stereo ${ }_{10}$ & $x$ & 1.47 & 8.43 & 16.35 & 3.06 & 13.36 & 22.26 & 10.82 & 30.25 & 29.17 & 17.37 & 39.49 & 62.19 \\
\hline
\end{tabular}

Note: $S_{g_{1}, g_{2}}$ is the proposed test with the first partition (deviance) into $g_{1}$ and the second partition into $g_{2}$ groups; FH-Stereo ${ }_{G}$ is the Fagerland-Hosmer test with the fitted probabilities from the ordered stereotype model using $G$ groups; and $G=g_{1} \times g_{2}$. 


\begin{tabular}{|c|c|c|c|c|c|c|c|c|c|c|c|c|c|}
\hline \multirow{2}{*}{ Test } & \multirow{2}{*}{ Covariates } & \multicolumn{3}{|c|}{$n=100$} & \multicolumn{3}{|c|}{$n=200$} & \multicolumn{3}{|c|}{$n=400$} & \multicolumn{3}{|c|}{$n=800$} \\
\hline & & $\alpha: 1 \%$ & $5 \%$ & $10 \%$ & $1 \%$ & $5 \%$ & $10 \%$ & $1 \%$ & $5 \%$ & $10 \%$ & $1 \%$ & $5 \%$ & $10 \%$ \\
\hline \multirow{6}{*}{$\begin{array}{l}S_{2,3} \\
\text { FH-Stereo }_{6} \\
S_{2,4} \\
\text { FH-Stereo }_{8} \\
S_{2,5} \\
\text { FH-Stereo }_{10}\end{array}$} & \multirow{2}{*}{$x_{1} \sim \mathcal{N}(5,3)$} & 2.11 & 11.08 & 18.59 & 6.46 & 33.15 & 36.61 & 21.69 & 55.86 & 58.28 & 24.76 & 59.87 & 64.17 \\
\hline & & 1.11 & 6.35 & 12.85 & 2.31 & 9.97 & 17.58 & 8.11 & 22.69 & 23.12 & 13.02 & 29.66 & 49.26 \\
\hline & \multirow{2}{*}{$x_{2} \sim \operatorname{Bern}(0.5)$} & 5.25 & 10.35 & 22.21 & 16.09 & 30.95 & 43.78 & 53.99 & 52.11 & 69.63 & 61.53 & 55.84 & 86.65 \\
\hline & & 1.54 & 6.05 & 10.67 & 3.28 & 9.55 & 14.52 & 11.46 & 21.64 & 19.09 & 18.39 & 28.27 & 40.68 \\
\hline & \multirow{2}{*}{$x_{3} \sim \operatorname{Bern}(0.7)$} & 2.42 & 10.75 & 19.47 & 7.46 & 32.16 & 38.38 & 25.08 & 54.22 & 61.09 & 28.58 & 58.07 & 67.26 \\
\hline & & 1.08 & 6.55 & 12.35 & 2.26 & 10.32 & 16.86 & 7.9 & 23.46 & 22.13 & 12.68 & 30.64 & 47.13 \\
\hline & \multirow{2}{*}{$x_{1} \sim \mathcal{N}(5,3)$} & 4.26 & 11.35 & 22.09 & 13.11 & 33.9 & 43.45 & 44.05 & 57.09 & 69.06 & 50.15 & 61.1 & 85.97 \\
\hline FH-Stereo 6 & & 1.09 & 9.09 & 13.6 & 2.32 & 14.38 & 18.55 & 8.09 & 32.59 & 24.38 & 12.97 & 42.54 & 51.97 \\
\hline & \multirow{2}{*}{$x_{2} \sim \operatorname{Bern}(0.5)$} & 2.43 & 10.75 & 20.52 & 7.45 & 32.16 & 40.45 & 25.03 & 54.21 & 64.33 & 28.58 & 58.04 & 70.81 \\
\hline $\mathrm{FH}^{-S_{\text {Stereo }}} 8$ & & 0.75 & 6.45 & 12.35 & 1.61 & 10.18 & 16.88 & 5.69 & 23.11 & 22.16 & 9.15 & 30.26 & 47.26 \\
\hline$S_{2,5}$ & \multirow{2}{*}{$x_{3} \sim \operatorname{Bern}(0.2)$} & 2.45 & 11.28 & 20.55 & 7.49 & 33.74 & 40.51 & 25.23 & 56.83 & 64.48 & 28.77 & 60.92 & 70.92 \\
\hline $\mathrm{FH}-$ Stereo $_{10}$ & & 1.25 & 6.26 & 12.52 & 2.64 & 9.86 & 17.08 & 9.28 & 22.34 & 22.42 & 14.92 & 29.21 & 47.75 \\
\hline
\end{tabular}

Note: $S_{g_{1}, g_{2}}$ is the proposed test with the first partition (deviance) into $g_{1}$ and the second partition into $g_{2}$ groups; FH-Stereo ${ }_{G}$ is the Fagerland-Hosmer test with the fitted probabilities from the ordered stereotype model using $G$ groups; and $G=g_{1} \times g_{2}$.

\begin{tabular}{|c|c|c|c|c|c|c|c|c|c|c|c|c|c|}
\hline \multirow{2}{*}{ Test } & \multirow{2}{*}{ Covariates } & \multicolumn{3}{|c|}{$n=100$} & \multicolumn{3}{|c|}{$n=200$} & \multicolumn{3}{|c|}{$n=400$} & \multicolumn{3}{|c|}{$n=800$} \\
\hline & & $\alpha: 1 \%$ & $5 \%$ & $10 \%$ & $1 \%$ & $5 \%$ & $10 \%$ & $1 \%$ & $5 \%$ & $10 \%$ & $1 \%$ & $5 \%$ & $10 \%$ \\
\hline & \multirow{2}{*}{$x_{1} \sim \mathcal{N}(5,3)$} & 2.23 & 10.35 & 19.32 & 6.91 & 30.97 & 38.08 & 23.2 & 52.13 & 60.57 & 26.43 & 55.79 & 66.64 \\
\hline FH-Stereo 6 & & 1.45 & 5.75 & 12.55 & 3.05 & 9.07 & 17.08 & 10.7 & 20.53 & 22.39 & 17.16 & 26.88 & 47.68 \\
\hline & \multirow[b]{2}{*}{$x_{2} \sim \operatorname{Bern}(0.5)$} & 4.21 & 10.38 & 19.26 & 12.89 & 31.07 & 37.95 & 43.34 & 52.36 & 60.32 & 49.39 & 56.12 & 86.41 \\
\hline $\mathrm{FH}_{-\mathrm{Stereo}_{8}}$ & & 1.27 & 6.57 & 12.93 & 2.71 & 10.34 & 17.55 & 9.49 & 23.61 & 23.12 & 15.21 & 30.92 & 49.29 \\
\hline & \multirow{2}{*}{$x_{3} \sim \operatorname{Bern}(0.7)$} & 1.75 & 10.25 & 17.87 & 5.41 & 30.58 & 35.19 & 18.17 & 51.51 & 56.01 & 20.71 & 55.21 & 61.67 \\
\hline FH-Stereo $_{10}$ & & 0.83 & 6.75 & 12.35 & 1.82 & 10.62 & 16.84 & 6.39 & 24.09 & 22.14 & 10.23 & 31.54 & 47.22 \\
\hline$S_{2,3}$ & \multirow{2}{*}{$x_{1} \sim \mathcal{N}(5,3)$} & 2.85 & 10.95 & 19.15 & 8.76 & 32.69 & 37.75 & 29.47 & 55.04 & 60.07 & 33.61 & 58.98 & 66.14 \\
\hline FH-Stereo 6 & & 1.55 & 6.85 & 14.25 & 3.31 & 10.79 & 19.45 & 11.56 & 24.45 & 25.54 & 18.5 & 31.98 & 54.47 \\
\hline$S_{2,4}$ & \multirow{2}{*}{$x_{2} \sim \operatorname{Bern}(0.5)$} & 4.96 & 10.85 & 21.35 & 15.23 & 32.41 & 42.03 & 51.17 & 54.63 & 66.81 & 58.3 & 58.47 & 93.52 \\
\hline $\mathrm{FH}^{2}$ Stereo $_{8}$ & & 1.25 & 7.95 & 11.87 & 2.62 & 12.59 & 16.15 & 9.21 & 28.5 & 21.21 & 14.76 & 37.25 & 45.24 \\
\hline & \multirow{2}{*}{$x_{3} \sim \operatorname{Bern}(0.2)$} & 4.83 & 11.28 & 20.55 & 14.85 & 33.72 & 40.46 & 49.93 & 56.84 & 64.39 & 56.9 & 60.85 & 90.86 \\
\hline FH-Stereo ${ }_{10}$ & & 1.15 & 5.25 & 12.61 & 2.46 & 8.33 & 17.19 & 8.57 & 18.92 & 22.56 & 13.82 & 24.76 & 48.13 \\
\hline
\end{tabular}

Note: $S_{g_{1}, g_{2}}$ is the proposed test with the first partition (deviance) into $g_{1}$ and the second partition into $g_{2}$ groups; FH-Stereo ${ }_{G}$ is the Fagerland-Hosmer test with the fitted probabilities from the ordered stereotype model using $G$ groups; and $G=g_{1} \times g_{2}$. 
A.2.6. Omission of a continuous covariate (independent of the other covariates). Tables AXXII-AXXIV give power to detect the omission of a continuous main effect term when the data were simulated from Scenario 6 in Table IV with the response category of $q=3,4,5$, respectively.

A.2.7. Omission of an interaction term between two dichotomous covariates when all the covariates in the model are dichotomous. Tables AXXV-AXXVII give power to detect the missing interaction term between a two dichotomous covariates in a model in which all the variables are dichotomous. The data were simulated from Scenario 7 in Table IV with the response category of $q=3,4,5$, respectively.

Table AXXII. Power of the test (\%) for the detection of omission of a continuous covariates which has not interaction with the other covariates in the model (Scenario 6, Table IV) when the number of response categories is $q=3$.

\begin{tabular}{|c|c|c|c|c|c|c|c|c|c|c|c|c|c|}
\hline \multirow{2}{*}{ Test } & \multirow{2}{*}{ Covariates } & \multicolumn{3}{|c|}{$n=100$} & \multicolumn{3}{|c|}{$n=200$} & \multicolumn{3}{|c|}{$n=400$} & \multicolumn{3}{|c|}{$n=800$} \\
\hline & & $\alpha: 1 \%$ & $5 \%$ & $10 \%$ & $1 \%$ & $5 \%$ & $10 \%$ & $1 \%$ & $5 \%$ & $10 \%$ & $1 \%$ & $5 \%$ & $10 \%$ \\
\hline$S_{2,3}$ & & 6.62 & 15.35 & 48.58 & .31 & 39.11 & 55.25 & 57 & 52.46 & 65.1 & 29.21 & 54.08 & 70.42 \\
\hline $\mathrm{FH}^{-S t e r e o}{ }_{6}$ & & 6.84 & 14.95 & 38.58 & 7.16 & 30.35 & 39.65 & 14.75 & 31.15 & 42.85 & 15.45 & 35.49 & 43.59 \\
\hline$S_{2,4}$ & & 6.76 & 16.55 & 51.49 & 7.47 & 42.23 & 58.57 & 27.24 & 56.73 & 69.01 & 30.04 & 58.56 & 74.72 \\
\hline FH-Stereo 8 & 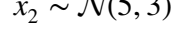 & 6.65 & 13.75 & 33.35 & 7.03 & 27.94 & 34.29 & 14.53 & 28.7 & 37.06 & 15.29 & 32.75 & 37.72 \\
\hline$S_{2,5}$ & & 7.05 & 15.35 & 50.94 & 7.81 & 39.15 & 57.95 & 28.48 & 52.57 & 68.33 & 31.39 & 54.21 & 73.93 \\
\hline $\mathrm{FH}-$ Stereo $_{10}$ & $1(0,10)$ & 6.72 & 14.06 & 31.95 & 7.07 & 28.62 & 32.84 & 14.62 & 29.45 & 35.58 & 15.33 & 33.65 & 36.26 \\
\hline
\end{tabular}

Note: $S_{g_{1}, g_{2}}$ is the proposed test with the first partition (deviance) into $g_{1}$ and the second partition into $g_{2}$ groups; FH-Stereo ${ }_{G}$ is the Fagerland-Hosmer test with the fitted probabilities from the ordered stereotype model using $G$ groups; and $G=g_{1} \times g_{2}$.

Table AXXIII. Power of the test (\%) for the detection of omission of a continuous covariates which has not interaction with the other covariates in the model (Scenario 6, Table IV) when the number of response categories is $q=4$.

\begin{tabular}{|c|c|c|c|c|c|c|c|c|c|c|c|c|c|}
\hline \multirow{2}{*}{ Test } & \multirow{2}{*}{ Covariates } & \multicolumn{3}{|c|}{$n=100$} & \multicolumn{3}{|c|}{$n=200$} & \multicolumn{3}{|c|}{$n=400$} & \multicolumn{3}{|c|}{$n=800$} \\
\hline & & $\alpha: 1 \%$ & $5 \%$ & $10 \%$ & $1 \%$ & $5 \%$ & $10 \%$ & $1 \%$ & $5 \%$ & $10 \%$ & $1 \%$ & $5 \%$ & $10 \%$ \\
\hline$S_{2,3}$ & & 5.62 & 17.33 & 49.58 & 6.27 & 44.09 & 56.42 & 22.79 & 59.17 & 66.5 & 25.06 & 61.05 & 71.94 \\
\hline $\mathrm{FH}^{-S_{t e r e o}} 6$ & $x_{1} \sim \jmath$ & 5.86 & 17.04 & 35.94 & 6.27 & 34.64 & 36.99 & 12.96 & 35.57 & 40.01 & 13.64 & 40.62 & 40.68 \\
\hline$S_{2,4}$ & & 7.71 & 21.32 & 43.16 & 8.56 & 54.28 & 49.17 & 31.14 & 72.86 & 58.03 & 34.24 & 75.21 & 62.84 \\
\hline $\mathrm{FH}^{-S_{t e r e O}} 8$ & $x_{2} \sim \mathcal{N}(5,3)$ & 6.52 & 18.84 & 35.54 & 6.88 & 38.25 & 36.54 & 14.22 & 39.35 & 39.51 & 14.94 & 44.85 & 40.27 \\
\hline$S_{2,5}$ & & 6.93 & 21.42 & 47.34 & 7.64 & 57.15 & 53.86 & 27.85 & 74.77 & 63.5 & 30.67 & 77.53 & 68.78 \\
\hline FH-Stereo $_{10}$ & $x_{2}$ & 7.12 & 21.13 & 34.82 & 7.49 & 42.85 & 35.81 & 15.46 & 44.04 & 38.76 & 16.28 & 50.26 & 39.46 \\
\hline
\end{tabular}

Note: $S_{g_{1}, g_{2}}$ is the proposed test with the first partition (deviance) into $g_{1}$ and the second partition into $g_{2}$ groups; FH-Stereo ${ }_{G}$ is the Fagerland-Hosmer test with the fitted probabilities from the ordered stereotype model using $G$ groups; and $G=g_{1} \times g_{2}$.

Table AXXIV. Power of the test (\%) for the detection of omission of a continuous covariates which has not interaction with the other covariates in the model (Scenario 6, Table IV) when the number of response categories is $q=5$.

\begin{tabular}{|c|c|c|c|c|c|c|c|c|c|c|c|c|c|}
\hline \multirow{2}{*}{ Test } & \multirow{2}{*}{ Covariates } & \multicolumn{3}{|c|}{$n=100$} & \multicolumn{3}{|c|}{$n=200$} & \multicolumn{3}{|c|}{$n=400$} & \multicolumn{3}{|c|}{$n=800$} \\
\hline & & $\alpha: 1 \%$ & $5 \%$ & $10 \%$ & $1 \%$ & $5 \%$ & $10 \%$ & $1 \%$ & $5 \%$ & $10 \%$ & $1 \%$ & $5 \%$ & $10 \%$ \\
\hline$S_{2,3}$ & & 6.82 & 18.83 & 42.26 & 7.58 & 48.06 & 48.07 & 7.61 & 64.53 & 56.69 & 30.4 & 66.58 & 61.36 \\
\hline FH-Stereo $_{6}$ & & 5.73 & 13.72 & 26.62 & 6.05 & 27.92 & 27.37 & 12.51 & 28.74 & 29.59 & 13.17 & 32.77 & 30.13 \\
\hline$S_{2,4}$ & & 6.92 & 23.66 & 36.94 & 7.73 & 60.36 & 42.01 & 28.16 & 81.02 & 49.51 & 30.98 & 83.57 & 53.58 \\
\hline FH-Stereo $_{8}$ & & 6.72 & 12.52 & 24.26 & 7.09 & 25.42 & 24.94 & 14.66 & 26.16 & 27.04 & 15.36 & 29.88 & 27.64 \\
\hline$S_{2,5}$ & $\sim \mathcal{U}(0,10)$ & 6.65 & 22.72 & 41.75 & 7.41 & 57.98 & 47.52 & 26.94 & 77.84 & 56.06 & 29.66 & 80.29 & 60.69 \\
\hline FH-Stereo $_{10}$ & $x_{3}$ & 6.14 & 16.11 & 22.91 & 6.45 & 32.83 & 23.58 & 13.35 & 33.71 & 25.53 & 14.03 & 38.52 & 26.06 \\
\hline
\end{tabular}

Note: $S_{g_{1}, g_{2}}$ is the proposed test with the first partition (deviance) into $g_{1}$ and the second partition into $g_{2}$ groups; FH-Stereo ${ }_{G}$ is the Fagerland-Hosmer test with the fitted probabilities from the ordered stereotype model using $G$ groups; and $G=g_{1} \times g_{2}$. 


\begin{tabular}{|c|c|c|c|c|}
\hline 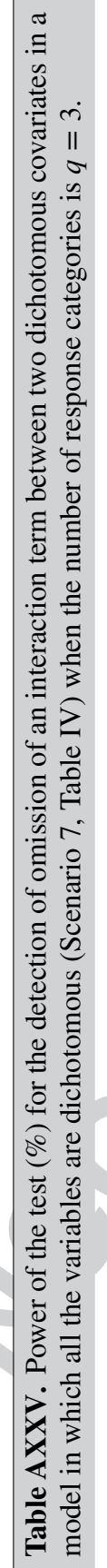 & 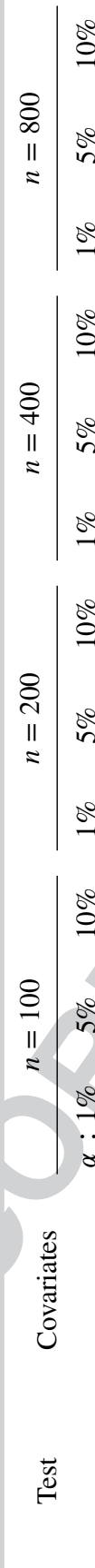 & 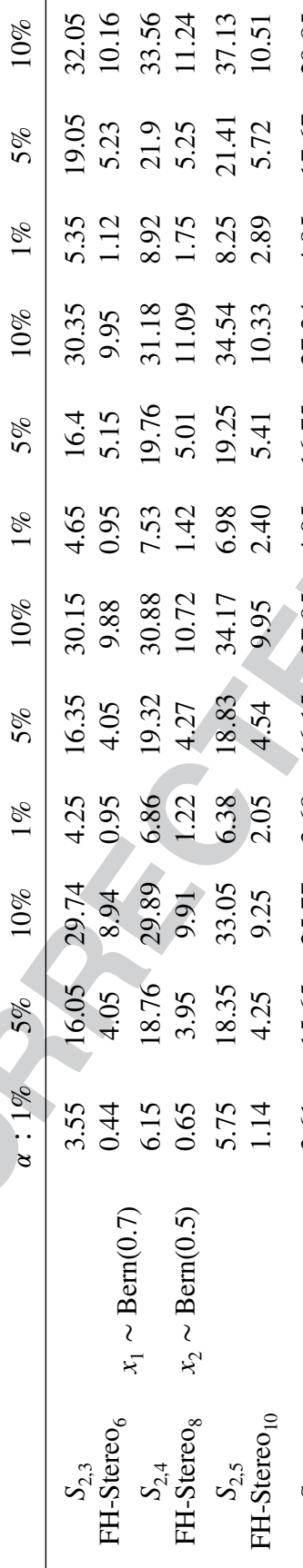 & 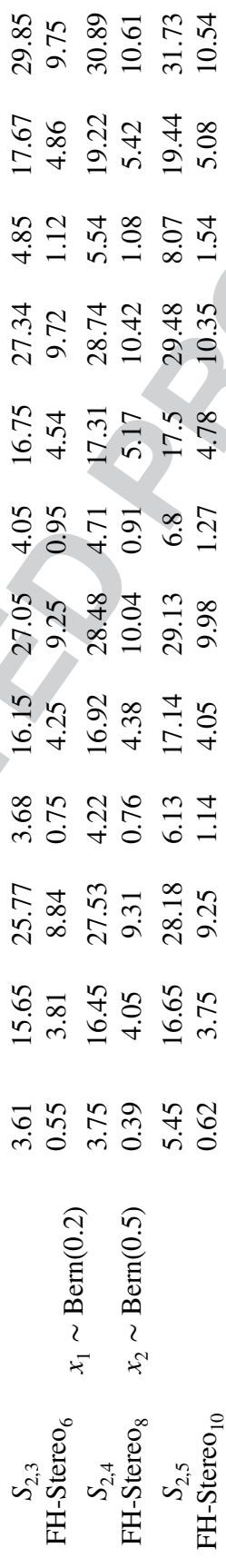 & 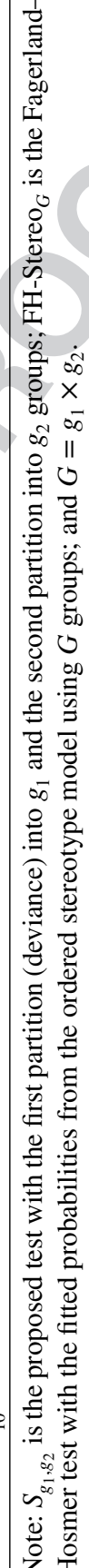 \\
\hline
\end{tabular}




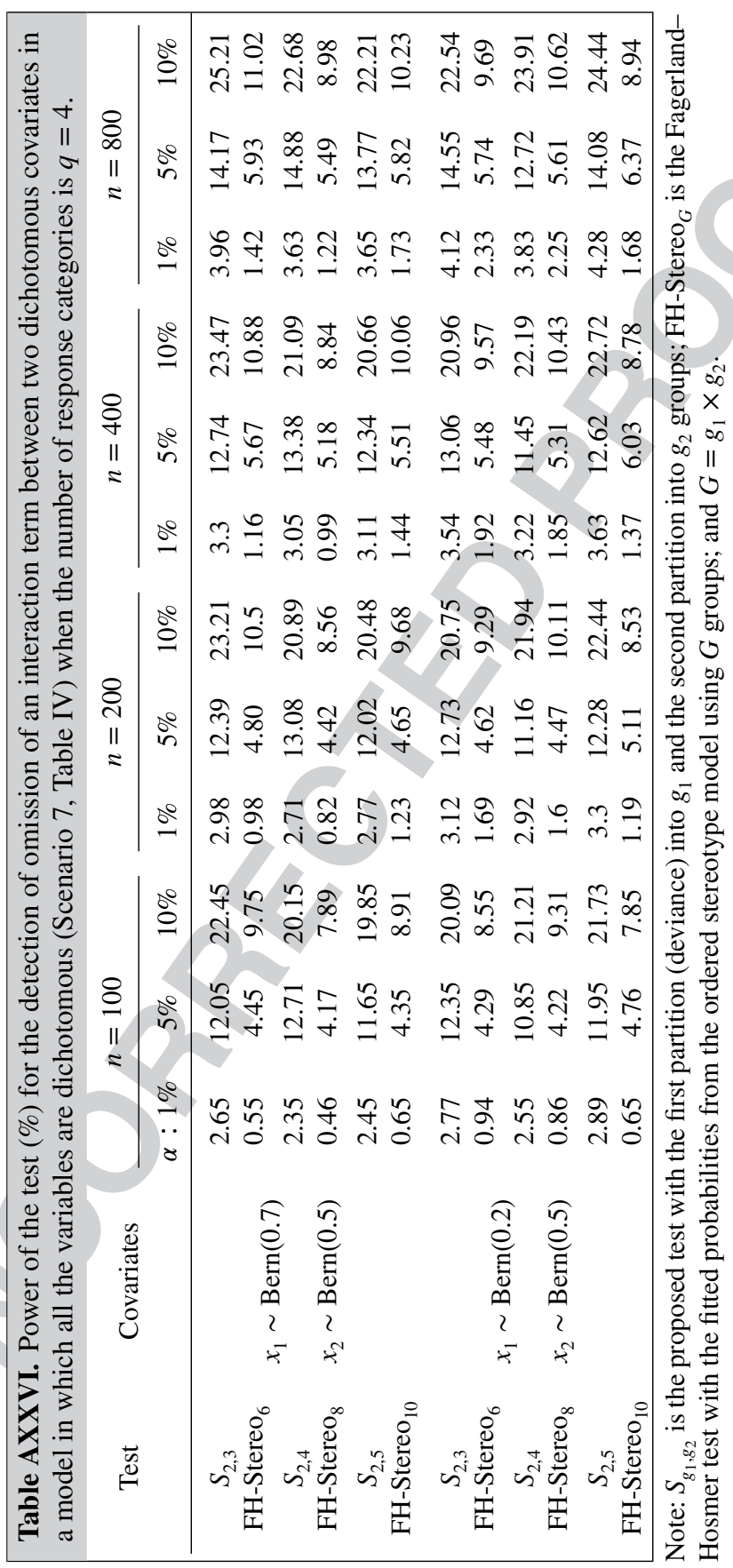




\begin{tabular}{|c|c|c|c|}
\hline $\begin{array}{l}\frac{\pi}{0} \\
\frac{0}{3} \\
\frac{\pi}{3} \\
\frac{\Xi}{8}\end{array}$ & 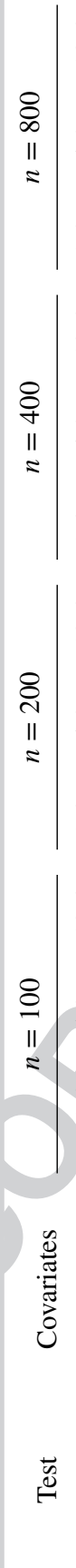 & $\stackrel{\circ}{\varrho}$ & 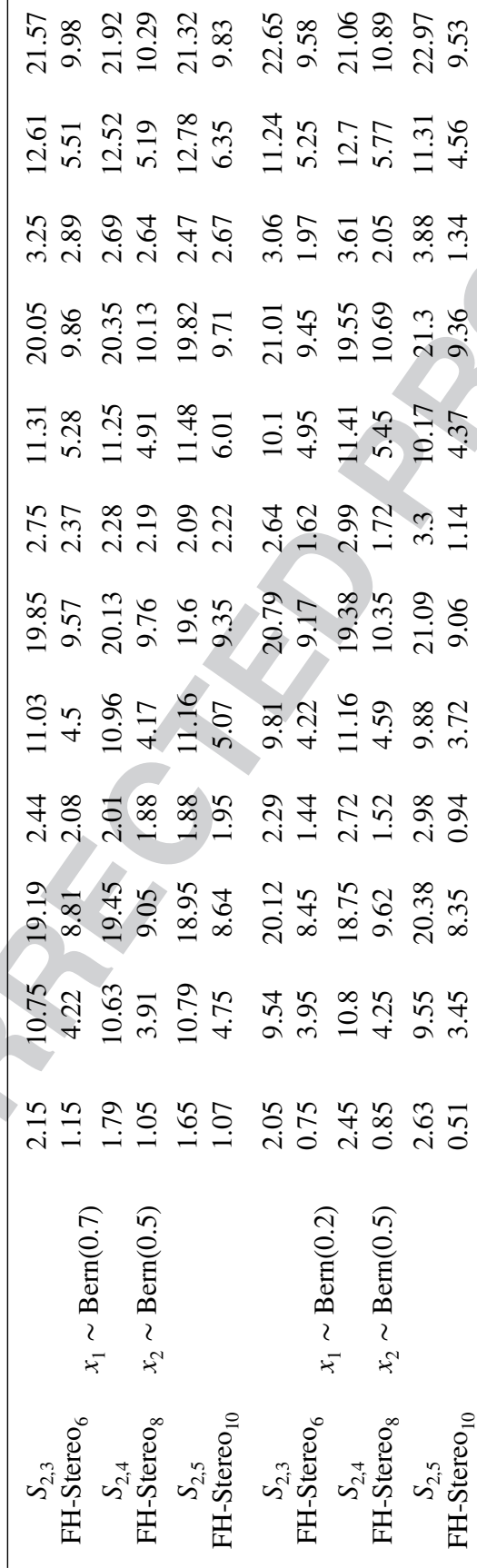 \\
\hline
\end{tabular}




\section{Appendix B : Applications}

\section{B.1. Example 1: Neuropsychiatric disturbance in aftercare placement}

Table BI shows the observed and estimated frequencies for the $S_{2,3}$ test and $G=6$ groups, following the fit in Table XVI.

\section{B.2. Example 2: Dangers in aftercare placement}

Table BI. Neuropsychiatric disturbance in aftercare placement data set: Observed and expected frequencies sorted according to the proposed partition scheme in Section 3 summed into $G=6$ groups in which all expected frequencies are greater than 1 and at least $80 \%$ are greater than 5 .

\begin{tabular}{|c|c|c|c|c|c|c|c|c|c|}
\hline \multirow{2}{*}{ Group } & \multicolumn{2}{|c|}{ Neuro $=1$} & \multicolumn{2}{|c|}{ Neuro $=2$} & \multicolumn{2}{|c|}{ Neuro $=3$} & \multicolumn{2}{|c|}{ Neuro $=4$} & \multirow{2}{*}{$\begin{array}{c}\text { Total } \\
(n=508)\end{array}$} \\
\hline & Obs. & Exp. & Obs. & Exp. & Obs. & Exp. & Obs. & Exp. & \\
\hline 1 & 62 & 60.76 & 11 & 10.24 & 5 & 5.03 & 7 & 8.96 & 85 \\
\hline 2 & 54 & 60.78 & 14 & 10.33 & 5 & 5.01 & 12 & 8.87 & 85 \\
\hline 3 & 54 & 60.12 & 16 & 10.18 & 7 & 4.94 & 7 & 8.76 & 84 \\
\hline 4 & 58 & 62.33 & 14 & 10.05 & 4 & 4.59 & 9 & 8.03 & 85 \\
\hline 5 & 62 & 61.95 & 13 & 10.16 & 4 & 4.69 & 6 & 8.21 & 85 \\
\hline 6 & 60 & 60.41 & 13 & 10.09 & 4 & 4.87 & 7 & 8.64 & 84 \\
\hline
\end{tabular}

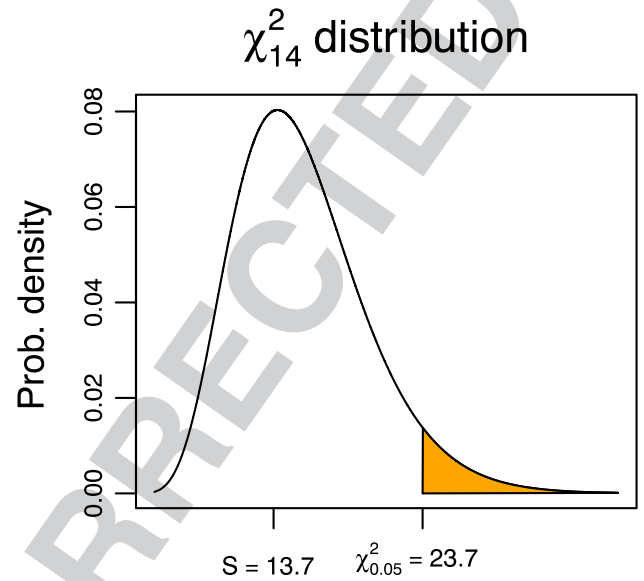

Figure B1. Neuropsychiatric disturbance in aftercare placement data set: Test statistic value under the null distribution that the stereotype model fits the data. On the graph, $\mathrm{S}$ is the value of the proposed test $S^{2,3}$ and $x_{0,05}^{2}$ is the 95 percentage point of the chi-squared distribution with $(G-2)(q-1)+(q-2)$ degrees of freedom.

\begin{tabular}{|c|c|c|c|}
\hline Type & Variable & Description & Values \\
\hline Response & Danger & Dangers the patient pose to others & $\begin{array}{l}1=\text { unlikely } \\
2=\text { possibly } \\
3=\text { probably } \\
4=\text { likely }\end{array}$ \\
\hline \multirow{4}{*}{ Covariates } & Gender & Gender of the adolescent & $\begin{array}{l}0=\text { female } \\
1=\text { male }\end{array}$ \\
\hline & Elope & Elopement risk & $\begin{array}{l}0=\text { no risk } \\
1=\text { history of risk }\end{array}$ \\
\hline & Behave & Behavioral symptom score & $0-9$ \\
\hline & $\begin{array}{l}\text { Age } \\
\text { LOS }\end{array}$ & \multicolumn{2}{|c|}{$\begin{array}{l}\text { Age in years, centered about the mean age } \\
\text { Length of stay in hospital, days }\end{array}$} \\
\hline
\end{tabular}




\begin{tabular}{|c|c|c|c|c|}
\hline Coefficient & Estimation & S.E. & $P$-value & 95\% C.I. \\
\hline$\widehat{\alpha}_{2}$ & -0.817 & 0.434 & & $(-1.668,0.034)$ \\
\hline$\widehat{\alpha}_{3}$ & -1.081 & 0.399 & & $(-1.863,-0.299)$ \\
\hline$\widehat{\alpha}_{4}$ & -1.275 & 0.441 & & $(-2.139,-0.411)$ \\
\hline$\widehat{\beta}_{1}$ (Gender) & 0.687 & 0.232 & 0.003 & $(0.232,1.142)$ \\
\hline$\widehat{\beta_{2}}$ (Elope) & -0.344 & 0.213 & 0.106 & $(-0.761,0.073)$ \\
\hline$\widehat{\beta}_{3}$ (Behave) & 0.840 & 0.079 & 0.000 & $(0.685,0.995)$ \\
\hline$\widehat{\beta}_{5}($ Age $)$ & -0.102 & 0.028 & 0.0003 & $(-0.157,-0.047)$ \\
\hline$\hat{\beta}_{6}(\operatorname{LOS})$ & 0.021 & 0.017 & 0.217 & $(-0.012,0.054)$ \\
\hline$\widehat{\phi}_{2}$ & 0.654 & 0.107 & & $(0.444,0.864)$ \\
\hline$\hat{\phi}_{3}$ & 0.854 & 0.106 & & $(0.646,1.062)$ \\
\hline
\end{tabular}

\begin{tabular}{|c|c|c|c|c|c|c|c|c|c|}
\hline \multirow{2}{*}{ Group } & \multicolumn{2}{|c|}{ Danger=Unlikely } & \multicolumn{2}{|c|}{ Danger=Possibly } & \multicolumn{2}{|c|}{ Danger=Probably } & \multicolumn{2}{|c|}{ Danger=Likely } & \multirow{2}{*}{$\begin{array}{c}\text { Total } \\
(n=508)\end{array}$} \\
\hline & Obs. & Exp. & Obs. & Exp. & Obs. & Exp. & Obs. & Exp. & \\
\hline 1 & 7 & 4.47 & 13 & 9.19 & 12 & 14.91 & 19 & 22.43 & 51 \\
\hline 2 & 8 & 5.56 & 9 & 8.81 & 12 & 14.60 & 22 & 22.03 & 51 \\
\hline 3 & 4 & 5.94 & 9 & 9.19 & 17 & 14.34 & 21 & 21.53 & 51 \\
\hline 4 & 3 & 3.50 & 10 & 8.83 & 17 & 15.34 & 21 & 23.33 & 51 \\
\hline 5 & 8 & 9.79 & 13 & 8.71 & 8 & 12.62 & 21 & 18.89 & 50 \\
\hline 6 & 3 & 7.68 & 11 & 8.66 & 15 & 13.82 & 22 & 20.84 & 51 \\
\hline 7 & 3 & 4.00 & 3 & 9.15 & 18 & 15.09 & 27 & 22.76 & 51 \\
\hline 8 & 7 & 4.75 & 6 & 8.44 & 16 & 14.94 & 22 & 22.87 & 51 \\
\hline 9 & 5 & 4.19 & 15 & 8.90 & 12 & 15.06 & 19 & 22.84 & 51 \\
\hline 10 & 6 & 7.84 & 12 & 9.22 & 14 & 13.24 & 18 & 19.70 & 50 \\
\hline
\end{tabular}

\section{Acknowledgements}

We thank Drs Shirley Pledger and Richard Arnold for their suggestions and stimulating discussions about this work. The authors are sincerely grateful to Morten W. Fagerland and David W. Hosmer for allowing us to use the determinants of aftercare placement data set. We are grateful to the two referees and the associate editor for helpful comments and references. This work was supported by the Marsden grant number E2317-2922.

\section{References}

1. Agresti A. Analysis of Ordinal Categorical Data 2nd ed., Wiley Series in Probability and Statistics. Wiley, 2010.

2. Stevens S. On the theory of scales of measurement. Science 1946; 103(2684):677-680.

3. Liu I, Agresti A. The analysis of ordered categorical data: an overview and a survey of recent developments. TEST: An Official Journal of the Spanish Society of Statistics and Operations Research 2005; 14(1):1-73.

4. McCullagh P. Regression models for ordinal data. Journal of the Royal Statistical Society 1980; 42:109-142.

5. McCullagh P, Nelder JA. Generalized Linear Models 2nd ed. Chapman \& Hall: London, 1989.

6. Peterson BL, Harrell FE. Partial proportional odds models for ordinal response variables. Applied Statistics 1990; 39: 205-217.

7. Anderson JA. Regression and ordered categorical variables. Journal of the Royal Statistical Society Series B 1984; 46(1): $1-30$.

8. Allison PD. Measures of fit for logistic regression. SAS Global Forum, Washington, DC, 2014.

9. Hosmer DW, Lemeshow S, Sturdivant RX. Applied Logistic Regression 3rd ed. Wiley: Hoboken, NJ, 2013.

10. Hosmer DW, Lemeshow S. Goodness of fit tests for the multiple logistic regression model. Communications in StatisticsTheory and Methods 1980; 9(10):1043-1069.

11. Fahrmeir L, Tutz G. Multivariate Statistical Modelling Based on Generalized Linear Models 2nd ed. Springer-Verlag: New York, 2001. 
12. Pulkstenis E, Robinson TJ. Goodness-of-fit tests for ordinal response regression models. Statistics in Medicine 2004; 23(6):999-1014.

13. Fagerland MW, Hosmer DW, Bofin AM. Multinomial goodness-of-fit tests for logistic regression models. Statistics in Medicine 2008; 27(21):4238-4253.

14. Lipsitz SR, Fitzmaurice GM, Molenberghs G. Goodness-of-fit tests for ordinal response regression models. Applied Statistics 1996:175-190.

15. Fagerland MW, Hosmer DW. A goodness-of-fit test for the proportional odds regression model. Statistics in Medicine 2013; 32(13):2235-2249.

16. Lin K-C, Chen Y-J. Assessing ordinal logistic regression models via nonparametric smoothing. Communications in Statistics - Theory and Methods 2008; 37:917-930.

17. Liu I, Mukherjee B, Suesse T, Sparrow D, Park SK. Graphical diagnostics to check model misspecification for the proportional odds regression model. Statistics in Medicine 2009; 28(3):412-429.

18. Li C, Shepherd BE. A new residual for ordinal outcomes. Biometrika 2012:1-8.

19. Greenland S. Alternative models for ordinal logistic regression. Statistics in Medicine 1994; 13(16):1665-1677.

20. Fernández D, Arnold R, Pledger S. Mixture-based clustering for the ordered stereotype model. Computational Statistics and Data Analysis 2016; 93:46-75. http://www.sciencedirect.com/science/article/pii/S016794731400317X.

21. R Development Core Team. R: A Language and Environment for Statistical Computing. R Foundation for Statistical Computing: Vienna, Austria, 2010. http://www.R-project.org, ISBN 3-900051-07-0.

22. Fontanella CA, Early TJ, Phillips G. Need or availability? modeling aftercare decisions for psychiatrically hospitalized adolescents. Children and Youth Services Review 2008; 30(7):758-773.

23. Haynam GE, Govindarajulu Z, Leone FC, Siefert P. Tables of the cumulative non-central chi-square distribution-part 11. Statistics: A Journal of Theoretical and Applied Statistics 1982; 13(3):413-443.

24. Tsiatis Anastasios A. A note on a goodness-of-fit test for the logistic regression model. Biometrika 1980; 67(1):250-251.

25. Pulkstenis E, Robinson TJ. Two goodness-of-fit tests for logistic regression models with continuous covariates. Statistics in Medicine 2002; 21(1):79-93.

26. Archer KJ, Lemeshow S, Hosmer DW. Goodness-of-fit tests for logistic regression models when data are collected using a complex sampling design. Computational Statistics \& Data Analysis 2007; 51(9):4450-4464.

27. Xie X-J, Pendergast J, Clarke W. Increasing the power: a practical approach to goodness-of-fit test for logistic regression models with continuous predictors. Computational Statistics \& Data Analysis 2008; 52(5):2703-2713.

28. Paul P, Pennell ML, Lemeshow S. Standardizing the power of the Hosmer-Lemeshow goodness of fit test in large data sets. Statistics in Medicine 2013; 32(1):67-80. 


\section{Author Query Form}

\section{Journal: Statistics in Medicine}

\section{Article: sim_7002}

Dear Author,

During the copyediting of your paper, the following queries arose. Please respond to these by annotating your proof with the necessary changes/additions.

- If you intend to annotate your proof electronically, please refer to the E-annotation guidelines.

- If you intend to annotate your proof by means of hard-copy mark-up, please use the standard proofreading marks in annotating corrections. If manually writing corrections on your proof and returning it by fax, do not write too close to the edge of the paper. Please remember that illegible mark-ups may delay publication.

Whether you opt for hard-copy or electronic annotation of your proof, we recommend that you provide additional clarification of answers to queries by entering your answers on the query sheet, in addition to the text mark-up.

\begin{tabular}{|c|l|c|}
\hline Query No. & \multicolumn{1}{|c|}{ Query } & Remark \\
\hline Q1 & $\begin{array}{l}\text { AUTHOR: Please check that authors and their affiliations are } \\
\text { correct. }\end{array}$ & \\
\hline Q2 & $\begin{array}{l}\text { AUTHOR: Figure caption was taken from the S1 manuscript. } \\
\text { Please check. }\end{array}$ & \\
\hline Q3 & $\begin{array}{l}\text { AUTHOR: Please provide the city location of publisher for } \\
\text { Reference 1. }\end{array}$ & $\begin{array}{l}\text { AUTHOR: Please provide volume number for References 14 } \\
\text { and 18. }\end{array}$ \\
\hline Q4
\end{tabular}


Required software to e-Annotate PDFs: Adobe Acrobat Professional or Adobe Reader (version 7.0 or above). (Note that this document uses screenshots from Adobe Reader $\mathrm{X}$ )

The latest version of Acrobat Reader can be downloaded for free at: http://get.adobe.com/uk/reader/

Once you have Acrobat Reader open on your computer, click on the Comment tab at the right of the toolbar:

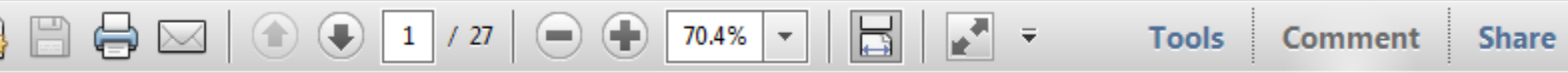

This will open up a panel down the right side of the document. The majority of tools you will use for annotating your proof will be in the Annotations section, pictured opposite. We've picked out some of these tools below:

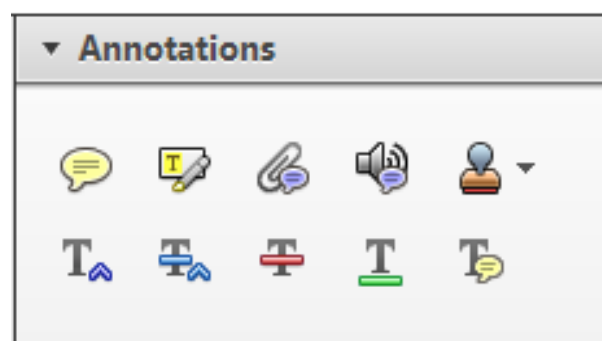

1. Replace (Ins) Tool - for replacing text.

Strikes a line through text and opens up a text box where replacement text can be entered.

How to use it

- Highlight a word or sentence.

- Click on the Replace (Ins) icon in the Annotations section.

- Type the replacement text into the blue box that appears.

Idard tramework for the analysis of $\mathrm{m}$ icy-Nevertheless, it also led to exog،

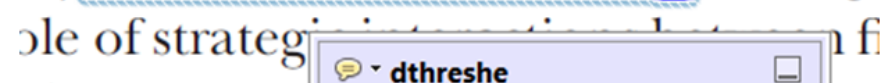
aber of comp 08/06/2011 15:58:17 is that the s1 nain compo: be level, are exc nc

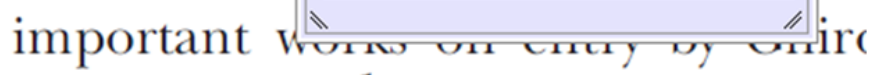
M heneferth) ${ }^{1}$ we anen the "hlarl $\mathrm{l}$

3. Add note to text Tool - for highlighting a section to be changed to bold or italic.

T Highlights text in yellow and opens up a text box where comments can be entered.

\section{How to use it}

- Highlight the relevant section of text.

- Click on the Add note to text icon in the Annotations section.

- Type instruction on what should be changed regarding the text into the yellow box that appears.

namic responses of mark ups ent with the VAR evidence

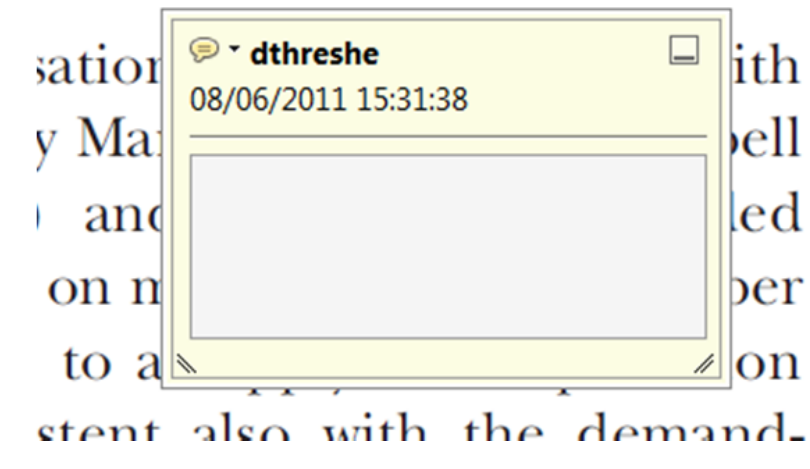

2. Strikethrough (Del) Tool - for deleting text.

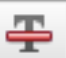

Strikes a red line through text that is to be deleted.

How to use it

- Highlight a word or sentence.

- Click on the Strikethrough (Del) icon in the Annotations section.

there is no room tor extra prohts al c ups are zero and the number of ret) values are not determined by Blanchard and Kiyotaki (1987), sfect competition in general equilil ts of aggregate demand and supply lassical framework assuming monol eph on evorenous number of firme

4. Add sticky note Tool - for making notes at specific points in the text.

Marks a point in the proof where a comment needs to be highlighted.

How to use it

- Click on the Add sticky note icon in the Annotations section.

- Click at the point in the proof where the comment should be inserted.

- Type the comment into the yellow box that appears.

iaisu airu suppiy sirucks. hivsl ui

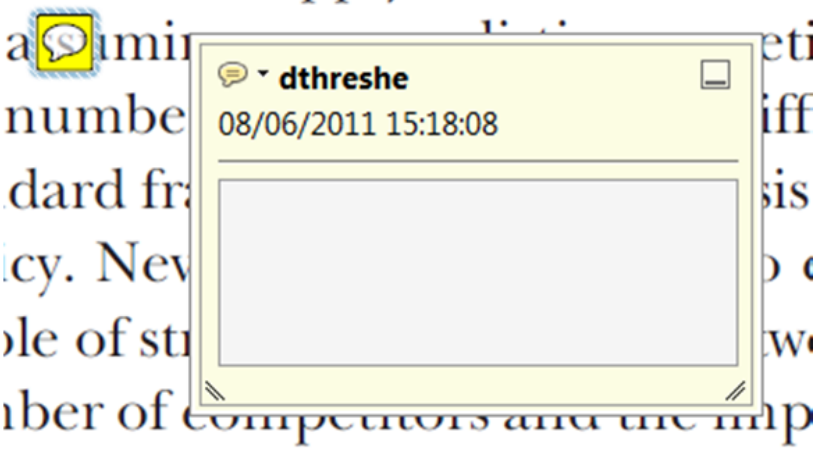

is that the structure of the secto. 
5. Attach File Tool - for inserting large amounts of text or replacement figures.

Inserts an icon linking to the attached file in the appropriate pace in the text.

How to use it

- Click on the Attach File icon in the Annotations section.

- Click on the proof to where you'd like the attached file to be linked.

- Select the file to be attached from your computer or network.

- Select the colour and type of icon that will appear in the proof. Click OK.

E N D

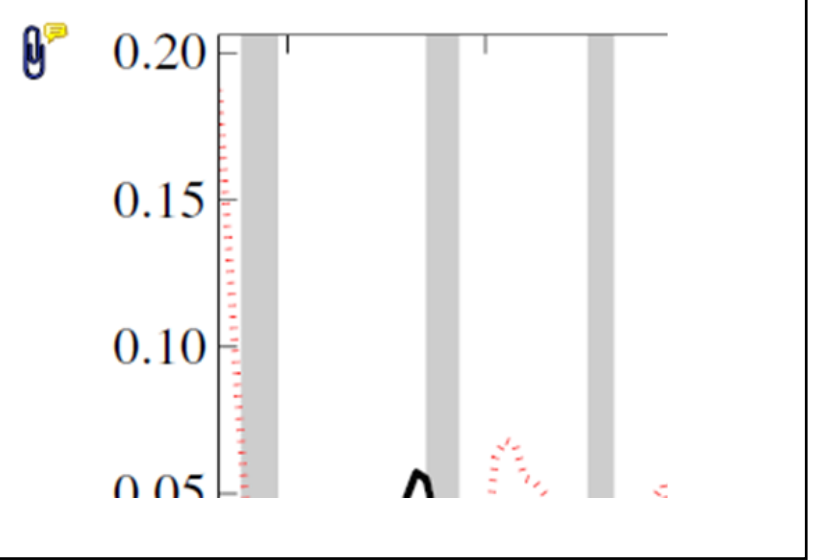

6. Add stamp Tool - for approving a proof if no corrections are required.

- Inserts a selected stamp onto an appropriate place in the proof.

\section{How to use it}

- Click on the Add stamp icon in the Annotations section.

- $\quad$ Select the stamp you want to use. (The Approved stamp is usually available directly in the menu that appears).

- Click on the proof where you'd like the stamp to appear. (Where a proof is to be approved as it is, this would normally be on the first page).

or the business cycie, starting with the on perfect competition, constant ret

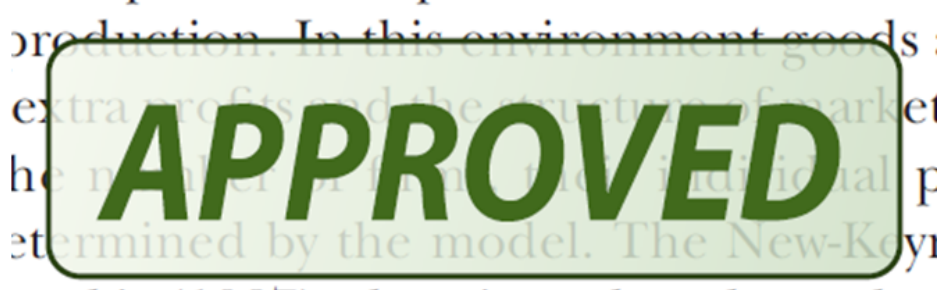
otaki (1987), has introduced produc general equilibrium models with nomin:

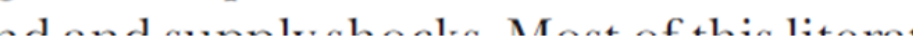

- Drawing Markups

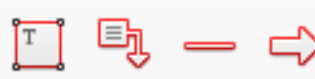

$0 \bigcirc \sqrt{6} \otimes$

\section{How to use it}

- Click on one of the shapes in the Drawing Markups section.

- Click on the proof at the relevant point and draw the selected shape with the cursor.

- To add a comment to the drawn shape, move the cursor over the shape until an arrowhead appears.

- Double click on the shape and type any text in the red box that appears.
7. Drawing Markups Tools - for drawing shapes, lines and freeform annotations on proofs and commenting on these marks.

Allows shapes, lines and freeform annotations to be drawn on proofs and for comment to be made on these marks.

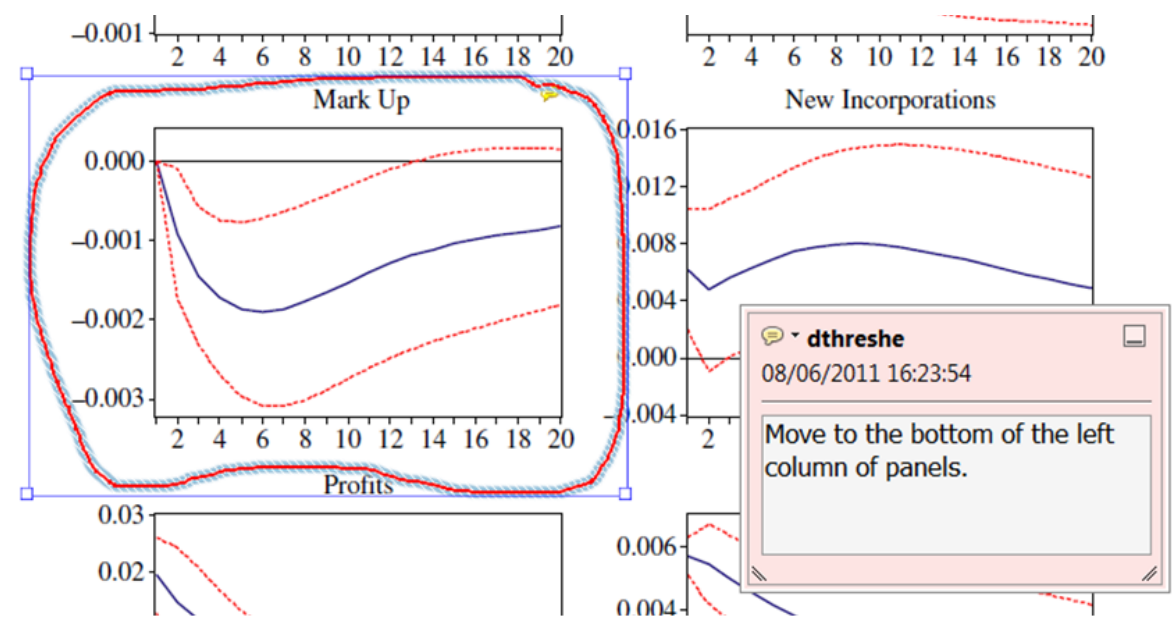

For further information on how to annotate proofs, click on the Help menu to reveal a list of further options:

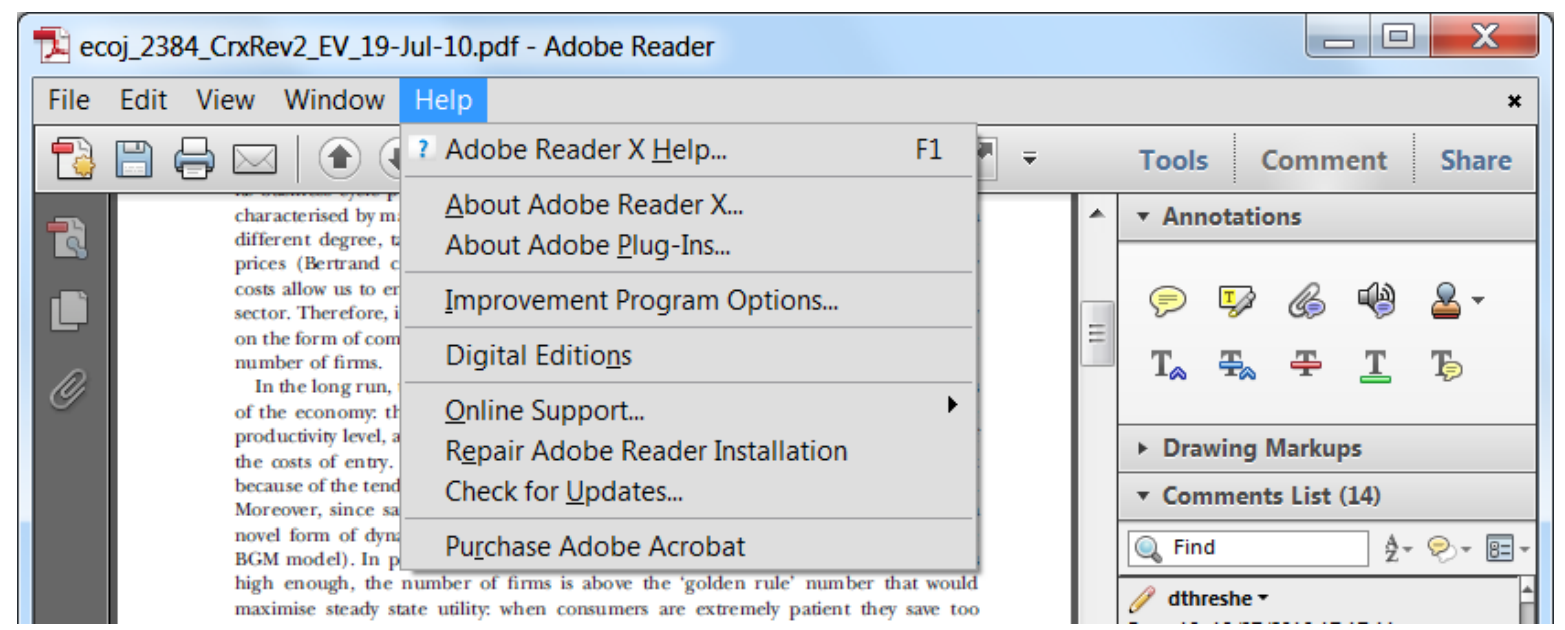

